\title{
Formulation of Metal-Organic Frameworks-based Drug Carrier by Controlled Coordination of Methoxy PEG Phosphate: Boosting Colloidal Stability and Redispersibility
}

Xu Chena, Yunhui Zhuang ${ }^{a}$, Nakul Rampala ${ }^{a}$, Rachel Hewitte, Giorgio Divitini ${ }^{b}$, Christopher A. $\mathrm{O}^{\prime} K^{2}{ }^{c}{ }^{c}$, Xiewen Liu ${ }^{a}$, Daniel J. Whitaker ${ }^{c}$, John W. Willse, Ravin Jugdaohsinghe, Jonathan J. Powelle, Han $\mathrm{Yu}^{\mathrm{d}, *}$, Clare P. Grey ${ }^{c}$, Oren A. Scherman ${ }^{c}$ and David Fairen-Jimenez ${ }^{\mathrm{a}, *}$

${ }^{a}$ Adsorption \& Advanced Materials Laboratory ( $\left.A^{2} M L\right)$, Department of Chemical Engineering \& Biotechnology, University of Cambridge, Philippa Fawcett Drive, Cambridge CB3 OAS, U.K. ${ }^{b}$ Electron Microscopy Group, Department of Materials Science and Metallurgy, University of Cambridge, 27 Charles Babbage Road, Cambridge CB3 OFS, U.K.

'Department of Chemistry, University of Cambridge, Lensfield Road, Cambridge CB2 1EW, U.K. dSchool of Chemical and Environmental Engineering Shanghai Institute of Technology, No. 100 Haiquan Road, Shanghai 201418, P.R. China

eBiominerals Research Laboratory \& Cellular Imaging and Analysis Facility, Department of Veterinary Medicine, University of Cambridge, Madingley Road, Cambridge CB3 OES, UK *e-mails: hanyu0220@tsinghua.edu.cn; df334@cam.ac.uk

\section{Table of Contents}

S1. General experimental remarks S2

S2. General synthetic procedures

S3 Characterizations

S4 BET area calculation using BETSI

S5. MD and GCMC simulations

s6. In Vitro evaluation of nanoMOFs

S7. References 


\section{S1. General experimental remarks}

Lyophilization was carried out using a Telstar LyoQuest benchtop freeze dryer (0.008 mBar, -70 $\left.{ }^{\circ} \mathrm{C}\right)$.

Powder X-ray Diffraction (PXRD): PXRD data were collected on a Bruker D8 DAVINCI diffractometer at $298 \mathrm{~K}$ using $\mathrm{Cu} \mathrm{K} \alpha$ radiation. The calculated PXRD patterns were produced using the Mercury program and single crystal reflection data.

Thermogravimetric Analysis (TGA): TGA measurements were carried out using a TA Instruments Q500 Thermogravimetric Analyzer. Measurements were collected from room temperature to $800{ }^{\circ} \mathrm{C}$ with a heating rate of $5^{\circ} \mathrm{C} / \mathrm{min}$ under nitrogen.

Fourier-transform Infrared Spectroscopy (FT-IR): FT-IR was carried out using a Bruker Tensor 27 FTIR with attenuated total reflectance (ATR) method.

Gas Uptake: $\mathrm{N}_{2}$ adsorption isotherm measurements were performed on a Micromeritics 3-Flex analyzer at $77 \mathrm{~K}$. Around $40 \mathrm{mg}$ samples were used for each measurement, Prior to the measurement, all the samples were degassed under vacuum at $55^{\circ} \mathrm{C}$ for 20 hours using the internal turbopump. $55^{\circ} \mathrm{C}$ was selected as the activation temperature to avoid further insertion of the PEG caused by thermal annealing, ${ }^{1}$ Gas uptakes were performed on the air-dried samples.

Dynamic Light Scattering (DLS) and Zeta Potential: Measurements were recorded in aqueous solution with the sample concentration of $0.2 \mathrm{mg} / \mathrm{mL}$ by a Zetasizer Nano ZS, (Malvern Instrument Ltd., U.K.) equipped with a He-Ne laser operating at $633 \mathrm{~nm}$ at $25^{\circ} \mathrm{C}$. Zeta potential was measured in a folded capillary Zeta cell DTS1070. The Smoluchowski equation was used to calculate the zeta potential. Measurements were performed three times with over 10 subruns for each sample. Error bars represent the standard deviation of three measurements.

Inductively Coupled Plasma-optical Emission Spectroscopy (ICP-OES): ICP-OES was performed using a Perkin Elemer ICP-OES Optima 2100DV. Samples were dispersed in $2 \mathrm{~mL}$ of nitric acid and $6 \mathrm{~mL}$ of hydrochloric acid (CAUTION!) and left to stand at room temperature in the fume cupboard for at least $1 \mathrm{~h}$ until all reactions have ceased. After that, samples were heated at $90{ }^{\circ} \mathrm{C}$ for $10 \mathrm{~h}$ to fully digest the sample. The mixture was diluted 750 times before the measurement.

X-ray Photoelectron Spectroscopy (XPS): XPS analysis was carried out using a Thermo Fisher Scientific ESCALAB 250Xi XPS System. A monochromatic Al-Ka source (1486.74 eV) and a charge neutralizer were used for all samples. Survey scans were acquired using pass energy of $100 \mathrm{eV}, 1$ scan and dwell time of $50 \mathrm{~ms}$. High-resolution data were acquired using $50 \mathrm{eV}$ pass energy, 50 
scans, dwell time of $50 \mathrm{~ms}$ and standard magnetic lenses.

UV-Vis Spectroscopy: UV-vis and fluorescence spectra were recorded using a Tecan Spark® Multimode Microplate Reader.

Liquid Nuclear Magnetic Resonance Spectroscopy (NMR) was carried out using a Bruker 400 $\mathrm{MHz}$ Avance III HD Smart Probe Spectrometer.

Solid-State Nuclear Magnetic Resonance Spectroscopy (NMR): Solid-state NMR experiments were conducted on a Bruker Avance III $200 \mathrm{MHz}$ spectrometer equipped with a $4.7 \mathrm{~T}$ magnet (corresponding to Larmor frequencies: $\mathrm{v}_{0}\left({ }^{1} \mathrm{H}\right)=200 \mathrm{MHz}$ and $\mathrm{v}_{0}\left({ }^{31} \mathrm{P}\right)=81.0 \mathrm{MHz}$ ). A Bruker $1.3 \mathrm{~mm}$ double-channel magic-angle spinning (MAS) probe was used with a spinning frequency of $60 \mathrm{kHz}$. Spectra were acquired using a rotor-synchronized Hahn echo experiment with optimized excitation and refocusing pulse lengths of 1.56 and $3.12 \mu \mathrm{s}$, respectively. A recycle delay of $1 \mathrm{~s}$ was used for all experiments, and either 4096 (mPEG-PO $\mathrm{PO}_{3}$ sample) or 8192 (MOF sample) transients were collected. Spectra were processed with $50 \mathrm{~Hz}$ of exponential apodization, zero filling, followed by Fourier transformation. ${ }^{31} \mathrm{P}$ chemical shifts were calibrated using $85 \% \mathrm{H}_{3} \mathrm{PO}_{4}$ in $\mathrm{H}_{2} \mathrm{O}\left(\delta_{\text {iso }}\left({ }^{31} \mathrm{P}\right)=0\right.$ ppm) as an external reference.

Scanning Electron Microscopy (SEM): The samples for SEM tests were coated with Pt or Au for 40 seconds and imaged using a FEI Nova Nano SEM 450.

Transmission Electron Microscopy (TEM): The samples for TEM test were prepared by dispersing the samples in ethanol using ultrasonication. After that, a small number of suspensions were dropcasted on a copper grid with a carbon support film. TEM micrographs were collected on a Tecnai F20 with an acceleration voltage of $200 \mathrm{kV}$. Scanning transmission electron microscopy (STEM) was carried out on a FEI Tecnai Osiris operated at $200 \mathrm{kV}$ using a OneView CMOS camera. The EDX chemical maps were acquired using a Super-X detector setup, with a total acquisition solid angle of $0.9 \mathrm{sr}$.

Cryogenic Electron Microscopy (cryo-EM): The samples were prepared for cryo-EM using a Vitrobot and conventional vitrification protocols (blot total 1, blot force 3, blot time $2.5 \mathrm{~s}$ ). The frozen samples on Quantifoil grids were imaged in a Krios cryo-EM operated at $300 \mathrm{kV}$, equipped with a Falcon4 detector. The images were acquired using EPU using a Falcon4 detector in counting mode.

Size Exclusion Chromatography with Multi-angle Light Scattering (SEC-MLAS) was performed on a Shimadzu HPLC system consisting of an LC-20AD Pump, SIL-20A autosampler, CTO-20A column oven and CBM-20A control unit. The column set was made up of 1 x PSS SUPREMA 
analytical $100 \AA(8 \times 300 \mathrm{~mm})$ and 2 x PSS SUPREMA analytical $3000 \AA$ ( 8 × $300 \mathrm{~mm})$. Dual detection was achieved via a Wyatt DAWN HELEOS-II multiangle light scattering (MALS) detector (laser at $\lambda$ $=658 \mathrm{~nm}$ ), and Wyatt Optilab rEX differential refractive index (DRI) detector with a $658 \mathrm{~nm}$ light source. MilliQ Water containing $0.1 \mathrm{~mol} / \mathrm{L}$ sodium nitrate and $0.01 \mathrm{~mol} / \mathrm{L}$ sodium azide was used as the eluent at a flow rate of $1.0 \mathrm{~mL} / \mathrm{min}$. The column temperature and the detector temperature were kept at $30^{\circ} \mathrm{C}$. All data analysis was performed using Wyatt Astra V 6.1.1 software. A literature value for the $\mathrm{dn} / \mathrm{dc}$ of poly(ethylene glycol) in water $(0.134 \mathrm{~mL} / \mathrm{g})^{2}$ was used to determine the molecular weight of all samples.

Redispersing of PEGylated nanoMOF was carried out using a ultrasonic sonicator bath (2 min, room temperature). Particle size distributions measured with DLS after low-speed centrifugation (1 $\mathrm{min}, 1000 \mathrm{rpm}$ ). For the convenience of observation, the process of redispersion, we used a probe sonicator (Qsonica Q125 sonicator with a $3 \mathrm{~mm}$ diameter probe) instead. Briefly, to a $20 \mathrm{~mL}$ vial, MOF@PEG-PO ${ }_{3}$ or DOX@MOF@PEG-PO $3(20 \mathrm{mg})$ and $\mathrm{H}_{2} \mathrm{O}(10 \mathrm{~mL})$ were added. The mixture was sonicated using a probe sonicator ( $20 \%$ amplitude) at room temperature for $2 \mathrm{~min}$, followed by standing for another 5 min. See Videos S2 and S3 in the Supporting Information.

Cell Culture: HeLa cells were chosen as our in vitro cellular system to evaluate the biocompatibility and cellular uptake efficiency of our nanoMOFs, as well as their capacity as drug delivery system. HeLa cells were maintained at $37{ }^{\circ} \mathrm{C}$ and $5 \% \mathrm{CO}_{2}$ in high rich glucose (4500 mg/L) DMEM ( Gibco Dulbecco's Modified Eagle Medium, Gibco $\left.{ }^{\circledR} 41965039\right)$ supplemented with $10 \%$ (v/v) fetal bovine serum (Sigma-Aldrich F9665), 100 units/mL penicillin, and $100 \mu \mathrm{g} / \mathrm{mL}$ streptomycin (Life Technologies 15140122). The supplemented medium is then named as 'complete' medium. PBS (Sigma D8537) and trypsin-EDTA (Life Technologies 25300054) were used to maintain the cell line.

MTS Cytotoxicity Assay: The concentration-dependent viability of PCN-128, PCN-128@PEG-PO PCN-222, PCN-222@PEG-PO 3 , DOX, DOX@PCN-128, DOX@128@PEG-PO 3 was investigated using the CellTiter $96 \circledast$ Aqueous Non-Radioactive Cell Proliferation Assay (Promega, UK). Briefly, HeLa cells were seeded on a 96-well plate at a density of 5000 cells/well for approximately $24 \mathrm{~h}$. Prior to each experiment, suspensions of PCN-128 vs PCN-128@PEG-PO, PCN-222 vs PCN222@PEG-PO $\mathrm{PO}_{3}$ (based on the nanoMOFs concentration) and DOX@PCN-128 and DOX@128@PEG-PO 3 (at a concentration of $1 \mathrm{mg} / \mathrm{mL}$ based on DOX) were freshly prepared before they were aliquoted in complete medium. Among them, suspensions of PCN-128@PEG-PO, PCN222 vs PCN-222@PEG-PO 3 DOX@128@PEG-PO 3 was prepared by redispersing the lyophilized samples in water. Then, nanoMOFs and drugs were dispersed in complete medium and a range of concentrations was prepared accordingly, of which $100 \mu \mathrm{L}$ were added to each well and incubated for $72 \mathrm{~h}$ at $37^{\circ} \mathrm{C}, 5 \% \mathrm{CO}_{2}$. At the end of the incubation period for $72 \mathrm{~h}$, the treatment solutions were removed, and cells were washed once with PBS. A solution of $100 \mu \mathrm{L}$ of complete media and $20 \mu \mathrm{L}$ 
of MTS solution was added to the wells, and the contents were incubated for $1.5 \mathrm{~h}$ at $37{ }^{\circ} \mathrm{C} / 5 \% \mathrm{CO}_{2}$. After the incubation, $100 \mu \mathrm{L}$ of the resulting solution was transferred to a clean 96-well plate, and the absorbance was recorded by UV-Vis SPECTROstar Nano at $490 \mathrm{~nm}$.

Flow Cytometry: HeLa cells were seeded on to a 6-well plate the day before they were treated with nanoMOFs at the nanoMOFs concentration of $10 \mu \mathrm{g} / \mathrm{mL}$ to each well. At the end of the incubation period, cells were gently lifted from the plate using $500 \mu \mathrm{L} /$ well of cell dissociation solution (Thermo Fisher Scientific, 13151014)) for $15 \mathrm{~min}$ at $37^{\circ} \mathrm{C} / 5 \% \mathrm{CO}_{2}$. The cell pellets were then collected and washed with PBS by centrifugation and stained with Live Dead stain (Thermo Fisher Scientific, L34955) on ice for 20 min according to the manufacturers' protocol. Following the staining, samples were washed with flow cytometry staining buffer (FACS buffer) and fixed on ice with $2 \%$ paraformaldehyde (PFA) for immediate acquisition on the same day. Unstained and single stain compensation tubes, with and without nanoMOFs treatment, were also prepared for spectral overlap compensation.

The CyAn ADP flow cytometer (Beckman Coulter, Ltd, High Wycombe, UK) is equipped with three lasers $(405,488$ and $642 \mathrm{~nm}$ ) in standard configuration and standard filter sets. Summit software v4.3 was used for acquisition and analysis (Beckman Coulter). Samples were filtered through $35 \mu \mathrm{m}$ nylon cell strainer mesh tubes (BD Biosciences) and 50,000 events were acquired for each sample.

Confocal Microscopy: HeLa cells were grown on $13 \mathrm{~mm}$ coverslips within a 24-well plate at $1.5 \times$ $10^{4}$ cells/well density for incubation overnight in complete medium at $37^{\circ} \mathrm{C} / 5 \% \mathrm{CO}_{2}$. Cells were then treated with $100 \mu \mathrm{g} / \mathrm{mL}$ of nanoMOFs diluted in media for $6 \mathrm{~h}$ or $24 \mathrm{~h}$ incubation. At the end of cellular incubations with or without MOFs, fluorescence labelling was carried out within the cell culture plate wells. In brief, cells were washed 3 times with PBS (5 min each). They were then fixed with $4 \%$ paraformaldehyde (PFA) for $10 \mathrm{~min}$, then washed 3 times (1 min each) in Tris-buffered saline (TBS). Following washing, fixed cells were stained with Wheat Germ Agglutinin (WGA) Alexa Fluor 555 conjugate (Thermo Fisher Scientific, W32464) for $15 \mathrm{~min}$ in TBS to visualize cell membranes. After 3 final washes in TBS, coverslips with cells attached were carefully removed from tissue culture plate wells and mounted on microscope slides using approximately $40 \mu \mathrm{L}$ mounting medium (Thermo Fisher Scientific, P36980).

Confocal imaging was carried out using a Zeiss LSM780 laser scanning confocal microscope. The $405 \mathrm{~nm}$ and $514 \mathrm{~nm}$ lasers were used to excite MOFs and WGA 555 stains, respectively. Images were collected using an oil immersion 63X/1.4NA lens. Zen software (Zeiss) was used for the acquisition image processing.

IncuCyte ${ }^{\circledR}$ Proliferation Assays: HeLa cells were seeded onto 96-well plates at a density of 1500 cells per well and incubated at $37^{\circ} \mathrm{C} / 5 \% \mathrm{CO}_{2}$ for approximately $24 \mathrm{~h}$. Then DOX, DOX@PCN-128, $\mathrm{DOX} @ 128 @ \mathrm{PEG}-\mathrm{PO}_{3}$ samples were freshly prepared and aliquoted in complete cell medium. Cells 
were then cultured and imaged within the IncuCyte Zoom (Essen BioScience) every $3 \mathrm{~h}$ using phasecontrast imaging with no lasers on. Images were exported and cells were semi-automatically quantified using ImageJ to find maxima with a range of noise tolerance level from 100-170. Finally, all images are compiled as image sequences to be exported as videos. See Videos S8-S10 in the Supporting Information.

Molecular Dynamics (MD) Simulations: The structure of PCN-222 is taken from the work of Feng

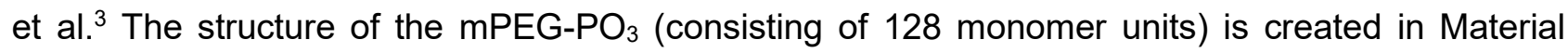
Studio. Geometry optimization of both the polymer and the framework is performed using the Forcite Module in Materials Studio. Charges for the framework and the PEG are calculated using the EQeq protocol. ${ }^{4}$ All simulations are performed using the LAMMPS ${ }^{5}$ molecular dynamics package using the Universal Force Field (UFF) ${ }^{6}$ parameters to describe the Lennard-Jones, bond, angle, dihedral and improper torsion potentials. The simulation box consists of a $2 \times 2 \times 2$ cell of PCN-222 - which is considered large enough to avoid any finite-size effects - and one mPEG-PO 3 chain placed at the external surface of the framework. Periodic boundary conditions are applied in all three dimensions. Initially, the energy minimization (equilibration run) of the model is done using the NVE ensemble until a temperature of $298 \mathrm{~K}$ is reached. Next, the production runs are conducted using the canonical ensemble (NVT) for a duration of $1.5 \mathrm{~ns}$. All MD simulations are done using a time step of $1 \mathrm{fs}$. The temperature is maintained using a Nosé-Hoover thermostat ${ }^{7}$ with a damping factor of $0.1 \mathrm{ps}$. The long range electrostatic interactions are computed using Ewald summation and the precision is set to $1 \times 10^{-4}$. The non-bonded van-der Waals interactions are computed using the Lennard-Jones potential and the short range electrostatic interactions are computed using the coulombic potential, both with a cut off of $12.5 \AA$. The trajectories are sampled every $0.01 \mathrm{~ns}$ and are viewed using VMD. ${ }^{8}$

Grand canonical Monte Carlo (GCMC) simulations: DOX has been modeled as a flexible model, except for the rings which are kept fixed. ${ }^{9}$ The bonding, bending, and torsional parameters have all been taken from the OPLS-AA force field. ${ }^{10}$ Table $\mathbf{S 5}$ shows the atom numbers assigned to the different atoms of the molecule, with their corresponding OPLS-AA atom types, along with their Lennard-Jones parameters, and charges. The loading of DOX at $1 \mathrm{~atm}$ and $298 \mathrm{~K}$ is simulated using the GCMC method as implemented in the RASPA simulation package. ${ }^{11}$ Monte Carlo moves consist of insertion, deletion, translation, and rotation moves, each taking place with equal probabilities. We use 20,000 cycles for equilibration and 200,000 cycles to average the properties. A cycle is defined as the maximum of 20 steps or the number of molecules in the system; this means that on average, a Monte Carlo move has been attempted on all the molecules during each cycle. The crystallographic information file (cif) of the different frameworks have been taken from the Cambridge structural database (CSD). ${ }^{12}$ All the framework atoms are kept fixed at the crystallographic positions with periodic boundary conditions applied in all directions to make sure that the simulation box is large enough to ensure that a distance of at least twice the cutoff radius is maintained between the periodic 
images. Intermolecular interactions are modeled using the Lennard-Jones (LJ) potential with a cutoff of $12.8 \AA$. Lorentz-Berthelot mixing rules are used for all the cross interaction terms. Electrostatic interactions are modeled using the coulombic potential and are computed using the Ewald summation method with the precision set to $10^{-6}$. The partial charges for the framework are calculated using the EQeq protocol. ${ }^{4}$ The framework atoms are modeled using LJ parameters taken from the DREIDING force field. ${ }^{13}$ For $\mathrm{Zr}$, which is not described in the DREIDING force field, the LJ parameters are taken from the Universal force field (UFF). ${ }^{6}$ Table S6 shows the LJ parameters for the atoms of the framework. Maximum DOX loadings (in wt\%) obtained from the GCMC simulations are listed in Table $\mathbf{S 4}$ with the corresponding snapshots showing the DOX (in green) loading within the framework shown in Figures S47 (MOF-808), S48 (NU-901), S49 (PCN-128), and S50 (PCN222). 


\section{S2. General synthetic procedures}

All reagents unless otherwise stated were purchased from Sigma Aldrich, Fluorochem or Alfa Aesar and used as provided. The synthesis of $Z r_{6}$ cluster, tetrakis (4-carboxyphenyl)porphyrin (TCPP) and (4', 4"', 4"'", 4'"'"'-(ethene-1,1,2,2-tet-rayl)tetrakis(([1,10-biphenyl]-4-carboxylate) (ETTC) were adapted from the literature procedures. ${ }^{3,14-15}$ Milli-Q water $\left(18.2 \mathrm{M} \Omega . \mathrm{cm}\right.$ resistivity at $\left.25^{\circ} \mathrm{C}\right)$ was used throughout the experiment. Dialysis tubing (Molecular Weight Cut-off; MWCO 3,500 and12,000 14,000 Daltons) was obtained from Medicell Membranes Ltd.

\section{Synthesis of mPEG5K-phosphate (mPEG-PO $\left.{ }_{3}\right)$}

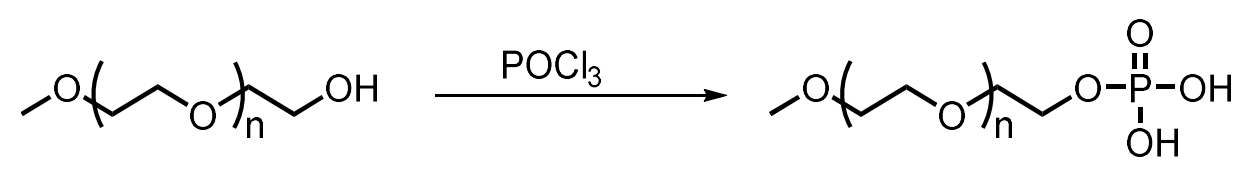

Scheme S1. Synthesis of mPEG-PO .

mPEG-PO ${ }_{3}$ was synthesized according to a previously reported method with slight modification. ${ }^{16}$ To a flame-dried two-neck flask, phosphorus oxychloride $\left(\mathrm{POCl}_{3}\right)$ in $(100 \mathrm{mmol}) 10 \mathrm{~mL}$ of dry DCM was added, followed by the addition of dry triethylamine $(300 \mathrm{mmol})$ at $0{ }^{\circ} \mathrm{C}$. After that, poly(ethylene glycol) methyl ether $\left(M_{n}=5000,30 \mathrm{mmol}\right)$ was added to the mixture in $100 \mathrm{~mL}$ of dry DCM over 30 $\mathrm{min}$. The resulting solution was stirred at room temperature for $10 \mathrm{~h}$, then $40 \mathrm{~mL}$ of water was added, the mixture was reacted for an additional $3 \mathrm{~h}$. DCM was removed under a vacuum, and the raw product was purified by dialysis (MWCO 3,500) followed by removing the residual water with lyophilization to give mPEG-PO ${ }_{3}$ as a white solid $(127.5 \mathrm{~g}, 25.5 \mathrm{mmol}, 85 \%)$. ${ }^{1} \mathrm{H}$ NMR $\left(\mathrm{D}_{2} \mathrm{O}, 400\right.$

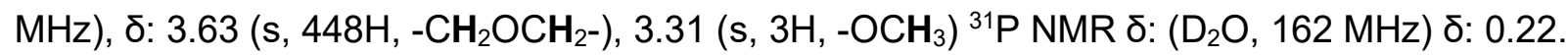

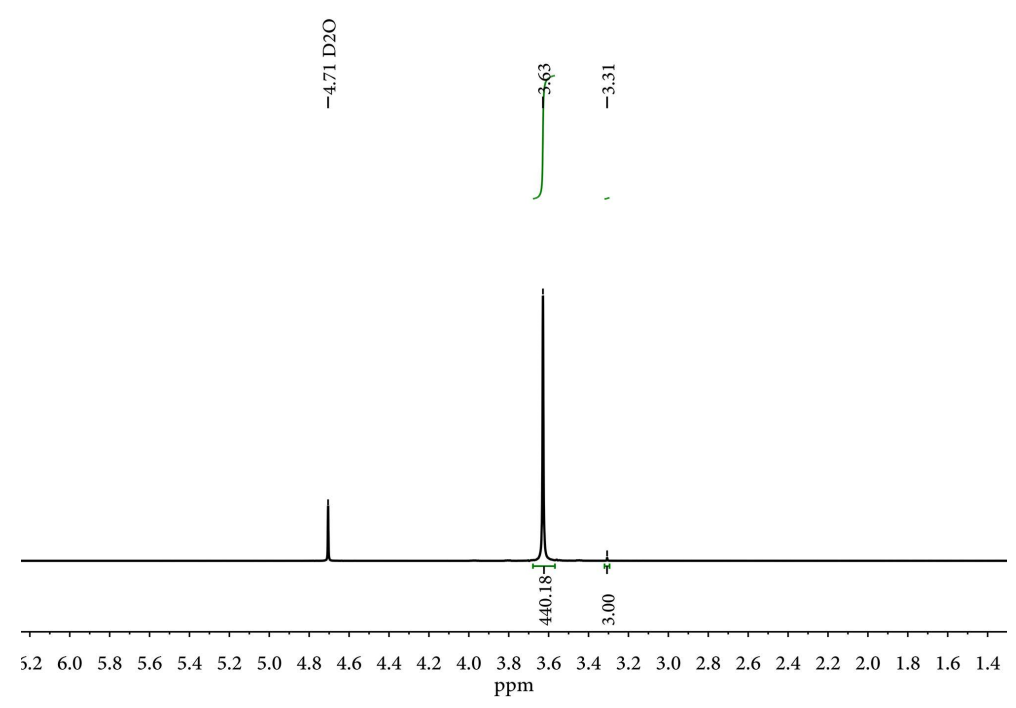

Figure S1. ${ }^{1} \mathrm{H}$ NMR spectrum of $\mathrm{mPEG}-\mathrm{PO}_{3}$. 


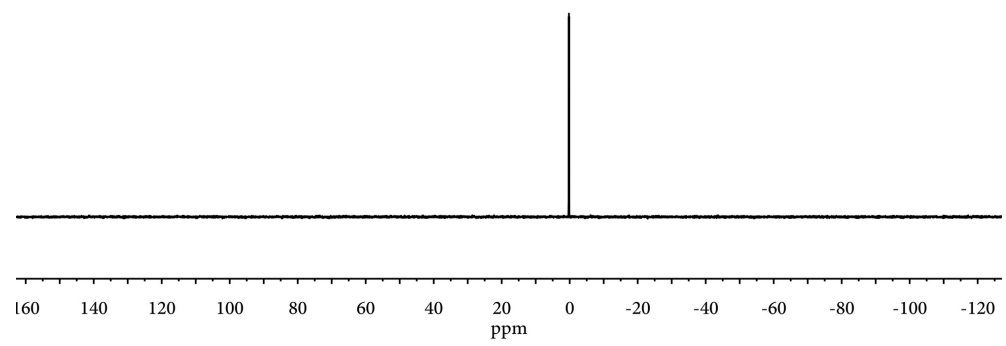

Figure S2. ${ }^{31} \mathrm{P} \mathrm{NMR}$ spectrum of $\mathrm{mPEG}-\mathrm{PO}_{3}$. Only one singlet was observed in ${ }^{31} \mathrm{P} \mathrm{NMR}$, indicating that either one or two PEG chains is/are attached to the phosphate group, whereas, $\mathrm{Mn}_{\mathrm{n}}$ obtained from GPC (Figure S3) further demonstrated the presence of one PEG chain.

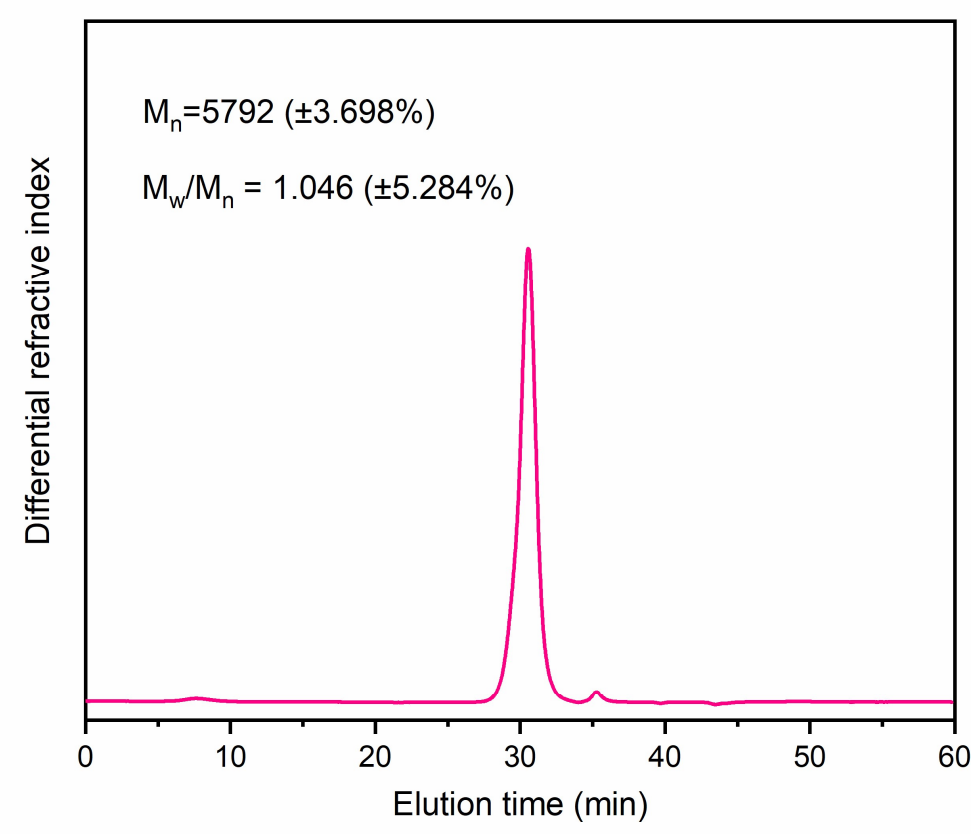

Figure S3. GPC trace of mPEG-PO $3(5 k)$.

\section{Size-Controlled Synthesis of PCN-222 Nanoparticles}

To control the size of PCN-222, different contents of trifluoroacetic acid (TFA) were utilized. The details are as follows:

TCPP (22.5 mg, $28.5 \mu \mathrm{mol}$ ), $\mathrm{Zr}_{6}$ cluster (38 mg, $\left.14.2 \mu \mathrm{mol}\right), 8 \mathrm{~mL}$ DMF and a $\mu \mathrm{L}$ of TFA were ultrasonically dissolved in a $20 \mathrm{~mL}$ threaded vial. The resultant mixture was placed in the $120{ }^{\circ} \mathrm{C}$ block heater for 5 hours. After cooling down to room temperature, the obtained purple sample was collected by high-speed centrifugation (15,000 rpm, $35 \mathrm{~min}$ ), followed by washing with fresh DMF 3 times and exchanging with ethanol 3 times. The final product was redispersed in ethanol for further use. $(a=80,130,180$ and $400 \mu \mathrm{L}$, the related size $=83.7 \pm 9.8 \mathrm{~nm}, 117.8 \pm 12.9 \mathrm{~nm}, 266.1 \pm 22.6$ $\mathrm{nm}$ and $628 \pm 36.6 \mathrm{~nm}$ ). 


\section{Synthesis of PCN-222 (Hf)}

TCPP (10 mg, $12.6 \mu \mathrm{mol}), \mathrm{HfOCl}_{2} \cdot 8 \mathrm{H}_{2} \mathrm{O}(25 \mathrm{mg}, 62.1 \mu \mathrm{mol}), 8 \mathrm{~mL}$ DMF and $200 \mu \mathrm{L}$ TFA were ultrasonically dissolved in a $20 \mathrm{~mL}$ threaded vial. The resultant mixture was placed in the $120{ }^{\circ} \mathrm{C}$ block heater for 5 hours. After cooling down to room temperature, the obtained purple sample was collected by high-speed centrifugation ( $15,000 \mathrm{rpm}, 35 \mathrm{~min}$ ), followed by washing with fresh DMF 3 times and exchanging with ethanol 3 times. The final product was redispersed in ethanol for further use.

\section{Synthesis of UiO-66 $(166.7 \pm 30.2 \mathrm{~nm})$}

1,4-benzenedicarboxylic acid (BDC) (320 mg, $1.93 \mathrm{mmol}$ ), $\mathrm{ZrCl}_{4}$ (466 mg, $2.00 \mathrm{mmol}$ ), benzoic acid $(2.44 \mathrm{~g}, 20 \mathrm{mmol})$ and $36 \mathrm{~mL}$ DMF were ultrasonically dissolved in a $100 \mathrm{~mL}$ threaded vial, followed by addition of concentrated $\mathrm{HCl}(0.33 \mathrm{~mL})$. The mixture was heated at $120^{\circ} \mathrm{C}$ for $48 \mathrm{~h}$. After cooling to room temperature, the obtained sample was collected by centrifugation $(4,700 \mathrm{rpm}, 35 \mathrm{~min})$, followed by washing with fresh DMF 3 times and exchanging with ethanol 3 times. The final product was redispersed in ethanol for further use.

\section{Synthesis of MOF-808 $(44.7 \pm 25.0 \mathrm{~nm})$}

1,3,5-benzenetricarboxylic acid (70 mg, $0.33 \mathrm{mmol}), \mathrm{ZrCl}_{4}(233 \mathrm{mg}, 1.00 \mathrm{mmol})$, formic acid (5.6 mL) and DMF $(10 \mathrm{~mL})$ were ultrasonically dissolved in a $50 \mathrm{~mL}$ threaded vial. The mixture was heated at $120^{\circ} \mathrm{C}$ for $48 \mathrm{~h}$. After cooling to room temperature, the obtained sample was collected by high-speed centrifugation (15,000 rpm, $35 \mathrm{~min}$ ), followed by washing with fresh DMF 3 times and exchanging with ethanol 3 times. The final product was redispersed in ethanol for further use.

\section{Synthesis of NU-901 $(251.2 \pm 47.1 \mathrm{~nm})$}

1,3,6,8-tetrakis ( $p$-benzoic acid)pyrene ( $\mathrm{H}_{4}$ TBAPy) $(25 \mathrm{mg}, 40.4 \mu \mathrm{mol}), \mathrm{ZrOCl}_{2} \cdot 8 \mathrm{H}_{2} \mathrm{O}$ (121 mg, 375.5 $\mu \mathrm{mol})$, 4-aminobenzoic acid (400 mg, $2.92 \mathrm{mmol})$, TFA $(200 \mu \mathrm{L})$ and DMF (20 mL) were ultrasonically dissolved in a $50 \mathrm{~mL}$ threaded vial. The mixture was heated at $140{ }^{\circ} \mathrm{C}$ for $50 \mathrm{~min}$, whereupon a yellow suspension formed. After cooling to room temperature, the obtained sample was collected by centrifugation (4,700 rpm, $35 \mathrm{~min}$ ), followed by washing with fresh DMF 3 times and exchanging with ethanol 3 times. The final product was redispersed in ethanol for further use.

\section{Synthesis of PCN-128 (180.9 $\pm 27.1 \mathrm{~nm})$}

ETTC (10 mg, $12.3 \mu \mathrm{mol}), \mathrm{Zr}_{6}$ cluster $(20 \mathrm{mg}, 7.5 \mu \mathrm{mol})$, DMF $(4 \mathrm{~mL})$ and TFA $(100 \mu \mathrm{L})$ were ultrasonically dissolved in a $6 \mathrm{~mL}$ threaded vial. Afterwards, triethylamine (TEA) $(20 \mu \mathrm{L})$ was added. The mixture was heated at $120^{\circ} \mathrm{C}$ for $24 \mathrm{~h}$. After cooling to room temperature, the obtained sample was collected by high-speed centrifugation $(15,000 \mathrm{rpm}, 35 \mathrm{~min})$, followed by washing with fresh DMF 3 times and exchanging with ethanol 3 times. The final product was redispersed in ethanol for further use. 


\section{The synthesis of PCN-222@PEG-PO 3}

mPEG-PO ${ }_{3}$ solution ( $10 \mathrm{~mL}, 25 \mathrm{mg} / \mathrm{mL}$ in $\mathrm{H}_{2} \mathrm{O}$ ) was added into the aqueous suspension of $\mathrm{PCN}-222$ $(5 \mathrm{~mL}, 10 \mathrm{mg} / \mathrm{mL})$. After stirring at room temperature for $16 \mathrm{~h}$, the reaction mixture was centrifuged to remove the unreacted $\mathrm{mPEG}-\mathrm{PO}_{3}$, and redispersed in water, then dialyzed (MWCO, 12,000 14,000) against water for $12 \mathrm{~h}$. The water was decanted and replenished with the fresh one every $4 \mathrm{~h}$. The obtained suspension of PCN-222@PEG-PO ${ }_{3}$ was then lyophilized $(0.008 \mathrm{mBar},-70$ ${ }^{\circ} \mathrm{C}$ ) to give a brown product, or collected by high-speed centrifugation (18,000 rpm, $35 \mathrm{~min}$ ), washed with ethanol and dried under the air, giving a dense and dark coloured product.

The methods for synthesis of UiO-66@PEG-PO 3 , MOF-808@PEG-PO, NU-901@PEG-PO 3 and PCN-128@PEG-PO ${ }_{3}$ were similar to that of PCN-222@PEG-PO ${ }_{3}$. The amount of mPEG-PO $\mathrm{P}_{3}$ was determined by ICP-OES (P/Zr). The amount of $\mathrm{mPEG}-\mathrm{PO}_{3}(\mathrm{wt} \%)=$ mass of encapsulated $\mathrm{mPEG}-$ $\mathrm{PO}_{3} / \mathrm{MOF} @ \mathrm{PEG}-\mathrm{PO}_{3} \times 100$.

\section{The time-dependent study of the PEGylation of PCN-222}

To 4 identical vials containing $5 \mathrm{~mL}$ water suspension of $120 \mathrm{~nm}$-sized PCN-222 $(10 \mathrm{mg} / \mathrm{mL})$ were added with $10 \mathrm{~mL}$ mPEG-PO 3 solution $\left(25 \mathrm{mg} / \mathrm{mL}\right.$ in $\mathrm{H}_{2} \mathrm{O}$ ), respectively, the mixture was stirred (800 $\mathrm{rpm}$ ) at room temperature for 2, 4, 12 and $16 \mathrm{~h}$. At each time point, one sample was quenched by centrifugation to remove the unreacted $\mathrm{mPEG}-\mathrm{PO}_{3}$, and washed with fresh water 3 times, then redispersed in water. The obtained suspension was lyophilized $\left(0.008 \mathrm{mBar},-70{ }^{\circ} \mathrm{C}\right)$ or collected by high-speed centrifugation (15,000 rpm, $35 \mathrm{~min}$ ), washed with ethanol and dried under the air, giving the desired products, named PEGylation $x \mathrm{~h}$ ( $x$ is the reaction time). The lyophilized sample was used for PXRD, EM imaging, while the air-dried sample was used for $\mathrm{N}_{2}$ adsorption measurements.

\section{DOX encapsulation}

DOX@MOF; The aqueous as-synthesized nanoMOFs $(5 \mathrm{mg} / \mathrm{mL}, 6 \mathrm{~mL})$ suspension was added to an aqueous DOX solution (15 mg/mL, $4 \mathrm{~mL}$ ), the mixture was then stirred for 48 hours at room temperature. Afterwards, the DOX loaded nanoMOFs were collected by centrifugation $(15,000 \mathrm{rpm}$, $35 \mathrm{~min}$ ) and washed with water for 3 times. The unloaded DOX was combined and diluted to a final volume of $500 \mathrm{~mL}$. The obtained DOX@MOF was dried under vacuum or redispersed in water for future use.

DOX@MOF@PEG-PO ${ }_{3}$; The aqueous as-synthesized nanoMOFs $(5 \mathrm{mg} / \mathrm{mL}, 6 \mathrm{~mL})$ suspension was added to an aqueous DOX solution (15 mg/mL, $4 \mathrm{~mL}$ ), the mixture was then stirred for 48 hours at room temperature. Afterwards, the aqueous $\mathrm{mPEG}-\mathrm{PO}_{3}$ solution $(6 \mathrm{~mL}, 25 \mathrm{mg} / \mathrm{mL}$ ) was added, the resulting mixture was stirred for another $16 \mathrm{~h}$. The reaction mixture was then centrifuged $(15,000$ $\mathrm{rpm}, 35 \mathrm{~min}$ ) to remove the unreacted $\mathrm{mPEG}-\mathrm{PO}_{3}$ and unloaded DOX, and redispersed in water, followed by dialysis (MWCO, 12,000 14,000) against water for $12 \mathrm{~h}$. The water was decanted and replenished with the fresh one every $4 \mathrm{~h}$. The unloaded DOX was combined and diluted to a final volume of $500 \mathrm{~mL}$. The final suspension was lyophilized $\left(0.008 \mathrm{mBar},-70^{\circ} \mathrm{C}\right)$ or dried under vacuum. 
The absorbance was determined by UV-Vis spectroscopy (maximum absorbance at $486 \mathrm{~nm}$ ). The concentration was calculated by comparing the UV-Vis absorbance with a calibration curve (Figure S31).

Drug loading $(w t \%)=$ (mass of drug used - mass of unloaded drug) $/$ (mass of MOF + loaded drug) $\times 100$.

\section{Drug release}

In PBS (pH = 7.4): Around 10 mg DOX@MOF and DOX@MOF@PEG-PO ${ }_{3}$ were suspended in 2 $\mathrm{mL}$ PBS $(\mathrm{pH}=7.4)$ and placed in dialysis bags (MWCO; 1,2000 - 1,4000) dispersed with $2 \mathrm{~mL}$ PBS $(\mathrm{pH}=7.4)$, dialyzing against the same media $(28 \mathrm{~mL})$ under magnetic stirring at room temperature. $1 \mathrm{~mL}$ of the media was taken out and replaced with fresh media at specific time intervals.

The drug released was analyzed by fluorescence spectroscopy by comparing with their calibration curves (Figure S32, emission maximum at $595 \mathrm{~nm}$ when excited at $486 \mathrm{~nm}$ ).

\section{Stability study}

DOX-loaded bare and PEGylated nanoMOFs were dispersed in PBS $(\mathrm{pH}=7.4)$ or water, samples nanoparticles were characterized by TEM and PXRD at different intervals of time. 


\section{S3. Characterizations}

\section{Optical and EM images}
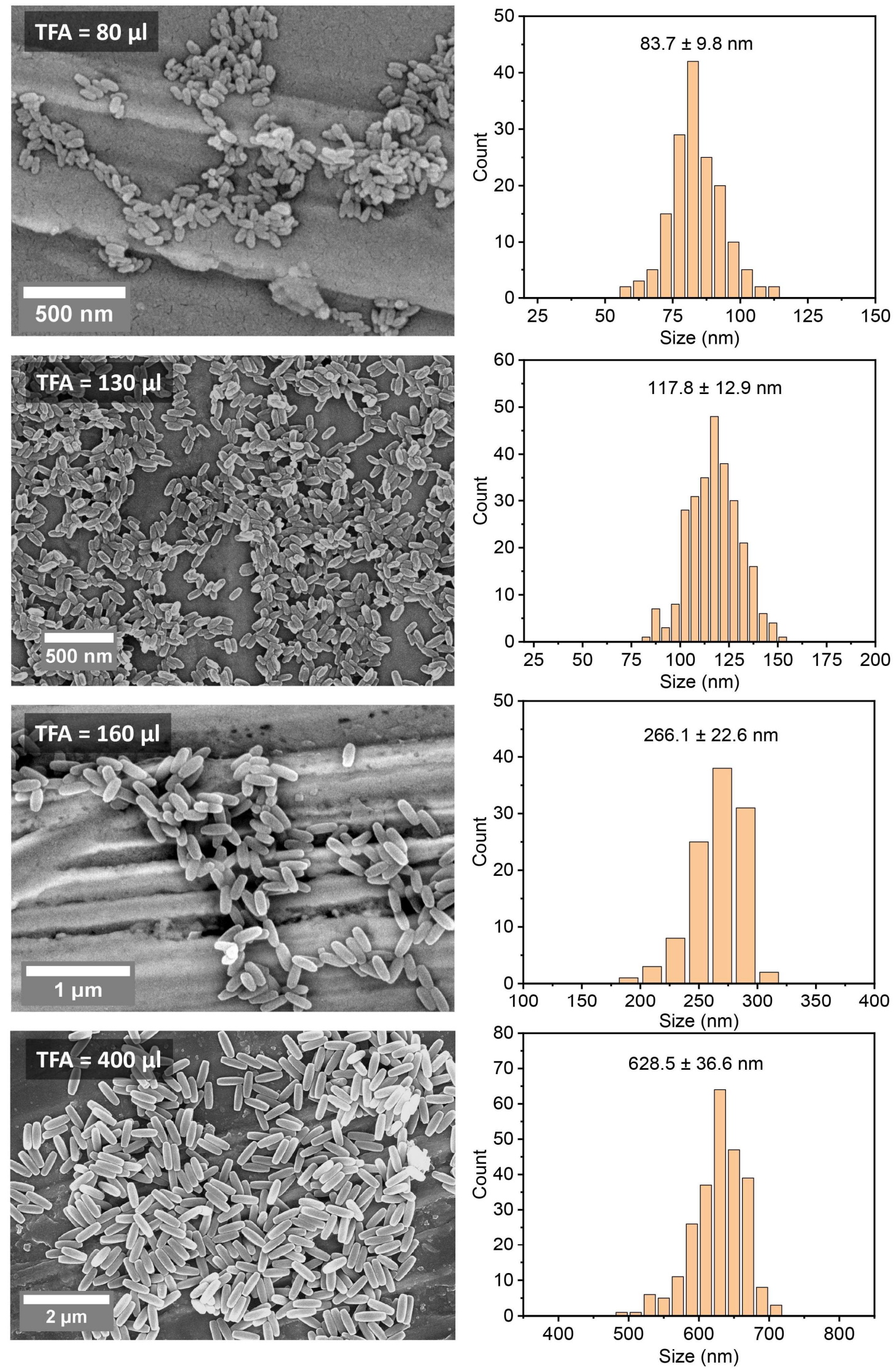

Figure S4. SEM images and particle size analysis of PCN-222. Average particle sizes were determined by manually counting at least 100 particles. 

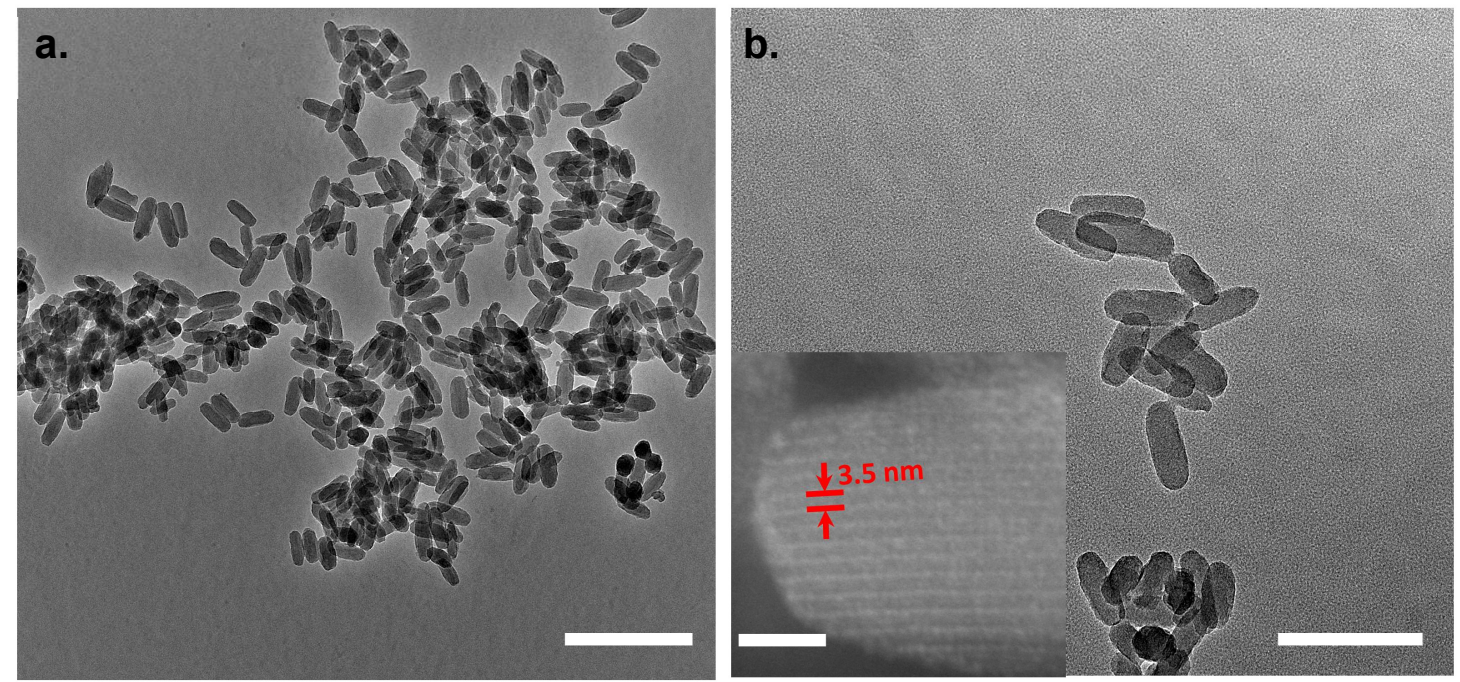

Figure S5. a. TEM and b. HAADF-STEM (inset) images of PCN-222. Scale bar, $500 \mathrm{~nm}$ (left), $200 \mathrm{~nm}$ (right) (inset, $15 \mathrm{~nm}$ ).

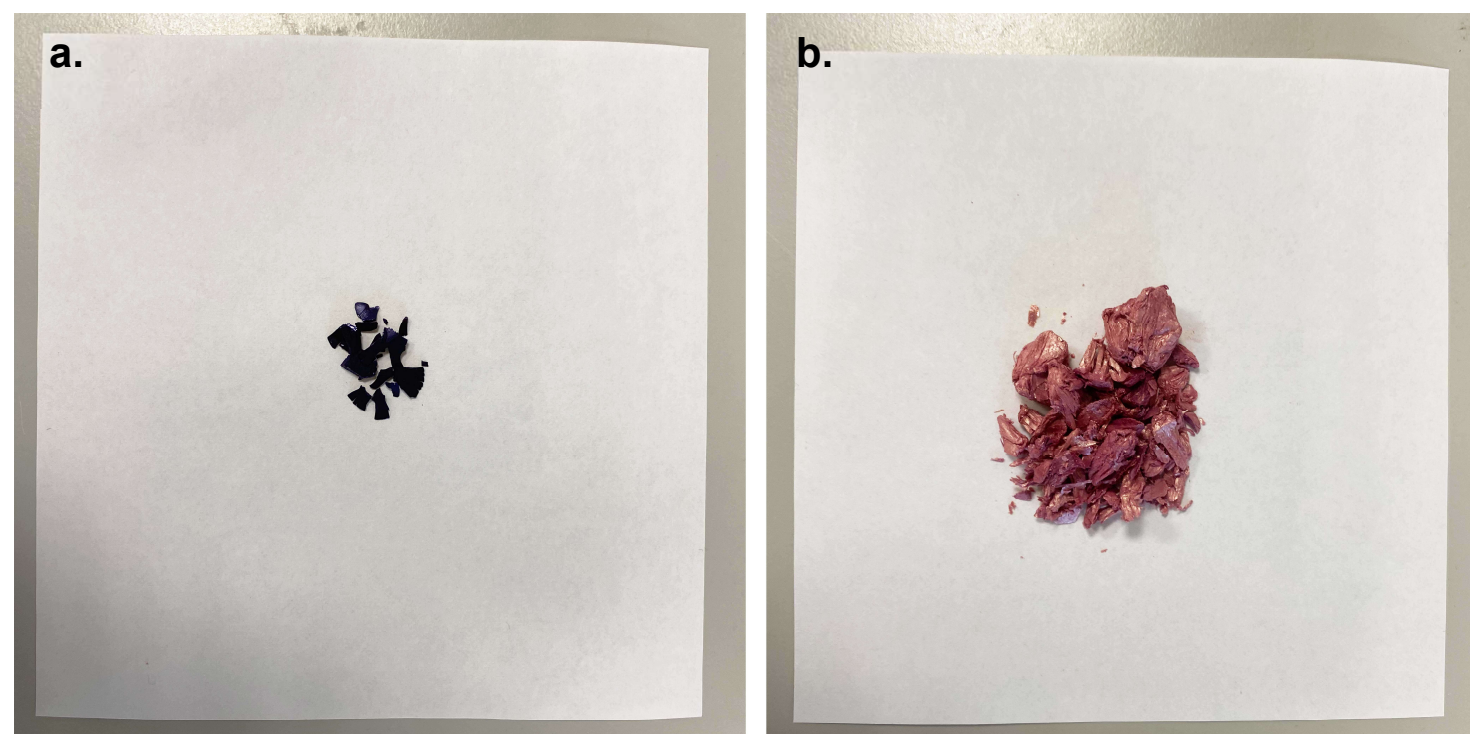

Figure S6. Photographs of a. ambient-dried PCN-222@PEG-PO ${ }_{3}$ and b. lyophilized PCN-222@PEG-PO 3 (both have the same mass, $60 \mathrm{mg}$ ).

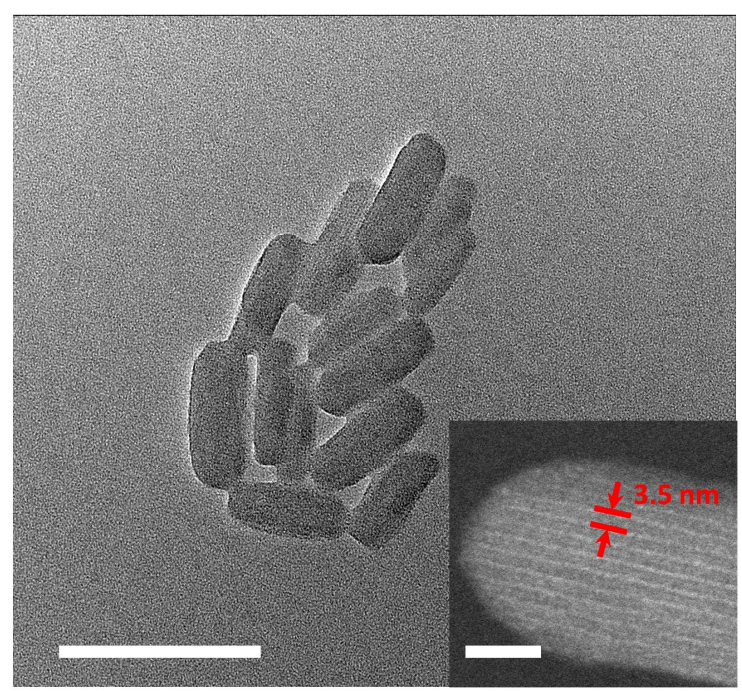

Figure S7. TEM and HAADF-STEM (inset) images of PCN-222@PEG-PO 3 . Scale bar, 200 nm (inset, 15 nm). 


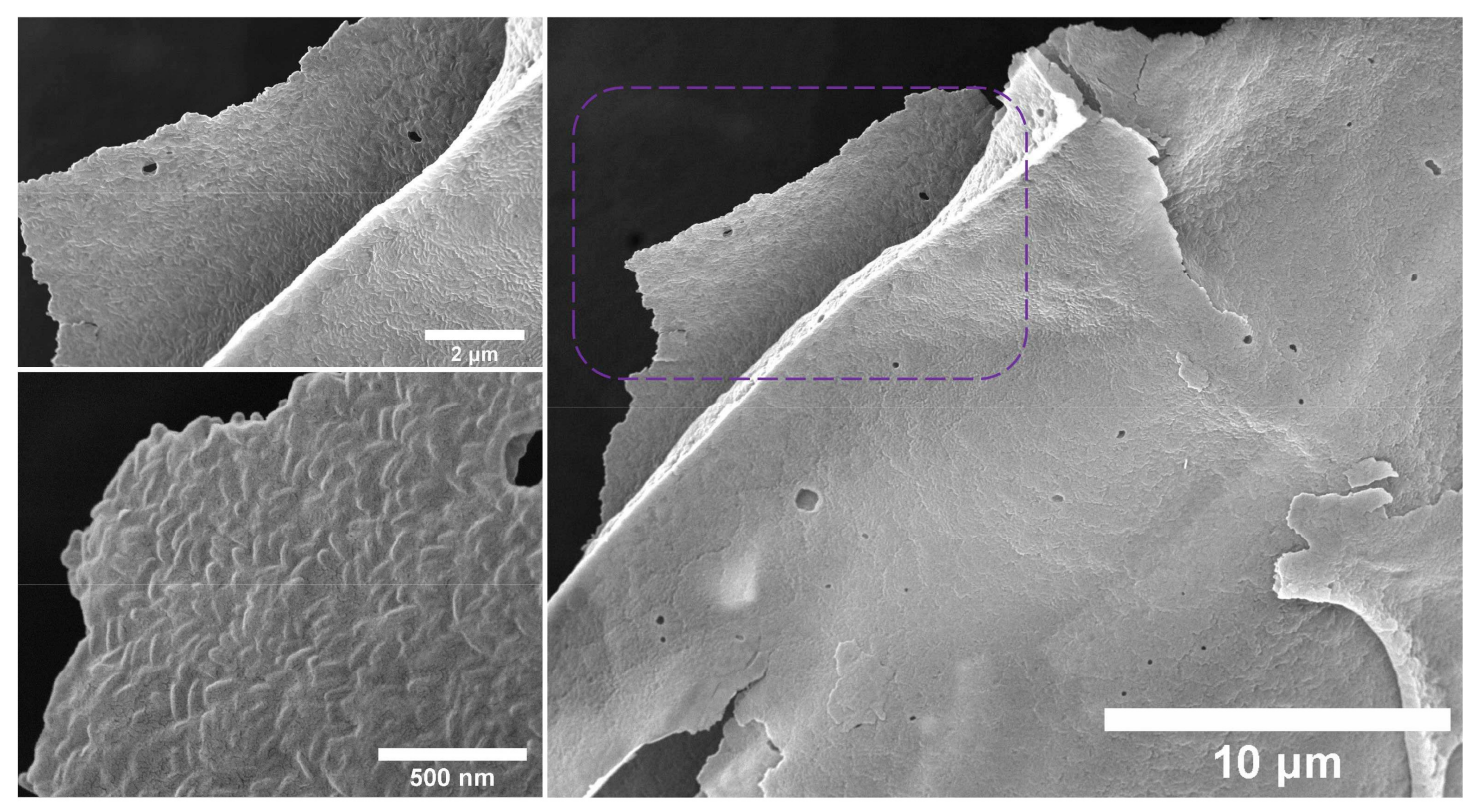

Figure S8. SEM images of lyophilized PCN-222@PEG- $\mathrm{PO}_{3}$ at different magnifications, clearly suggesting that the PCN-222@PEG-PO 3 are deeply immersed in the mPEG matrix.
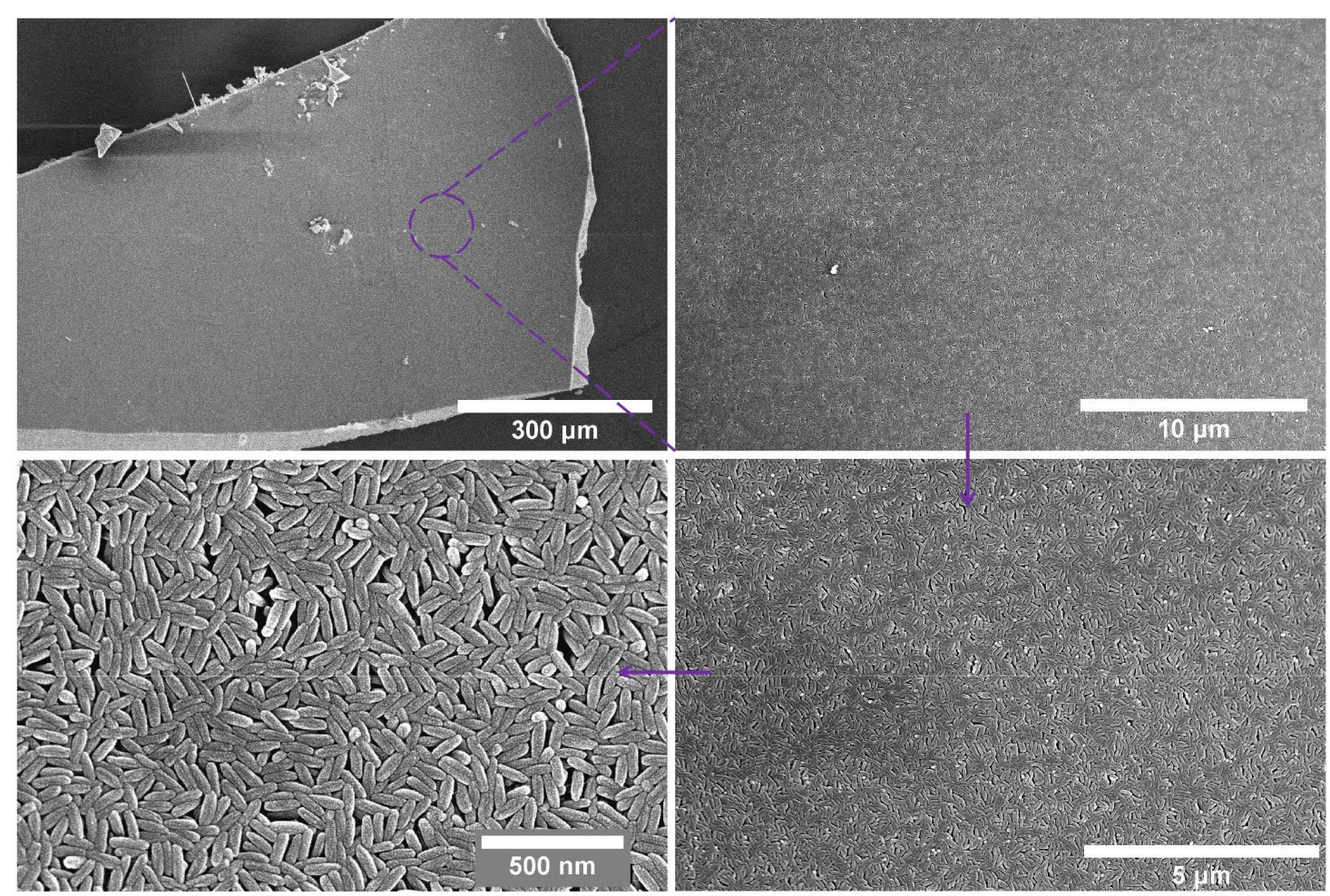

Figure S9. SEM images of ambient-dried PCN-222@PEG-PO ${ }_{3}$ with different magnifications, which clearly indicate the close packing and uniform dispersion of PCN-222@PEG-PO ${ }_{3}$ and their rod-shaped morphology. 

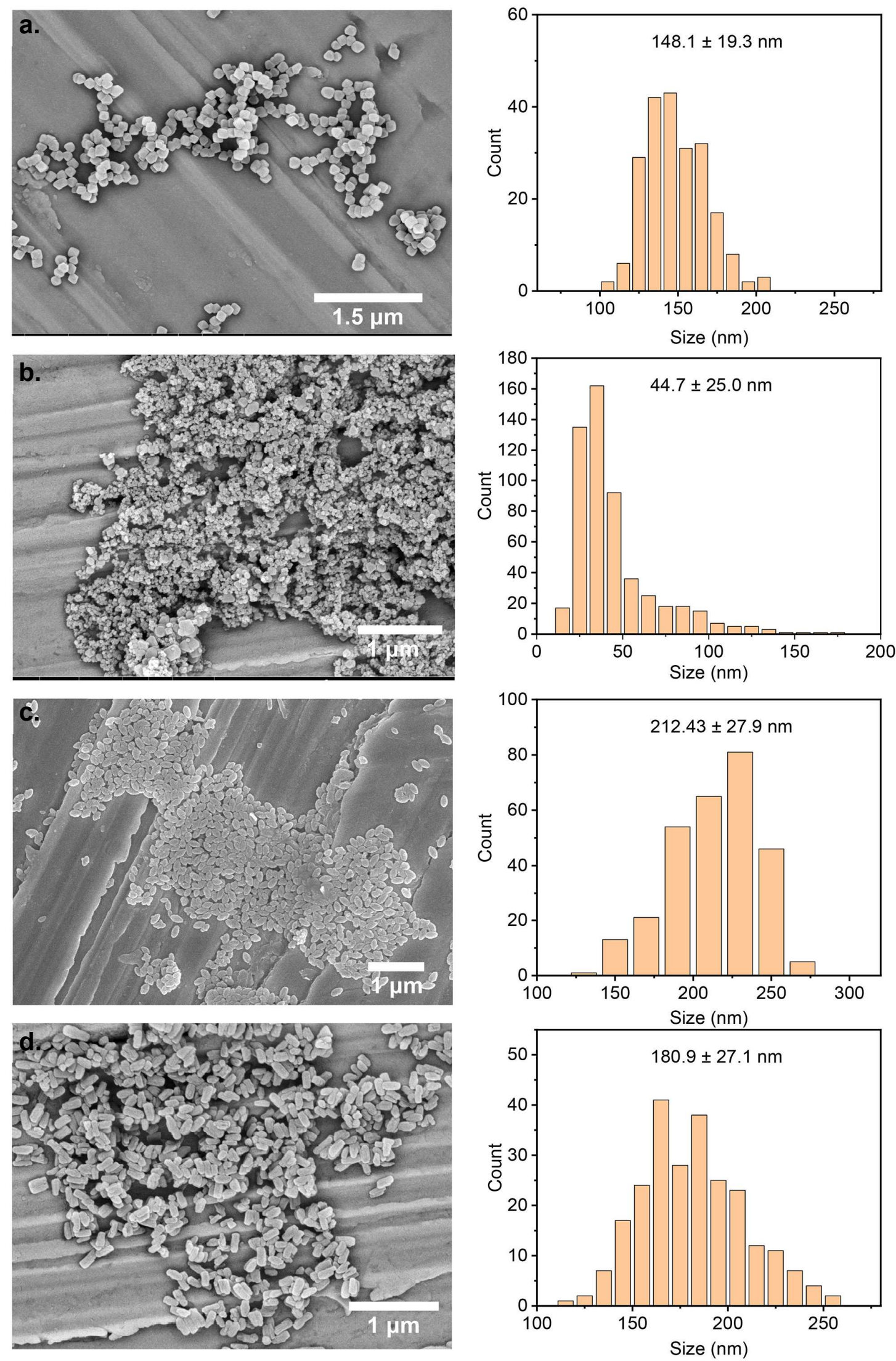

Figure S10. SEM images and particle size analysis of a. UiO-66, b. MOF-808, c. NU-901 and d. PCN-128. Average particle sizes were determined by manually counting at least 100 particles. 

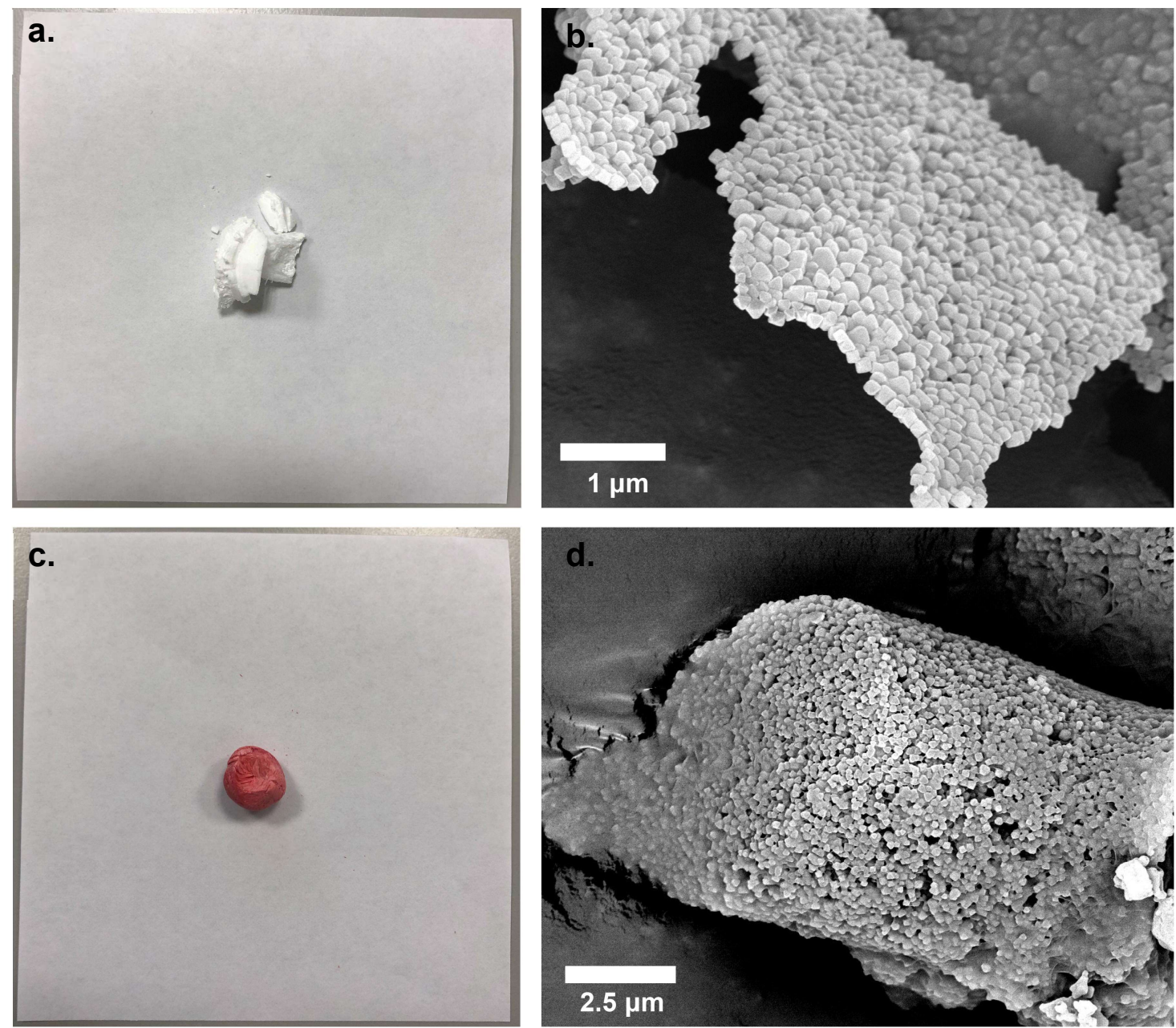

Figure S11. Morphological characterization. a. and c. Optical images, b. and d. SEM images of UiO-66@PEG$\mathrm{PO}_{3}$ and DOX@66@PEG-PO 3. 

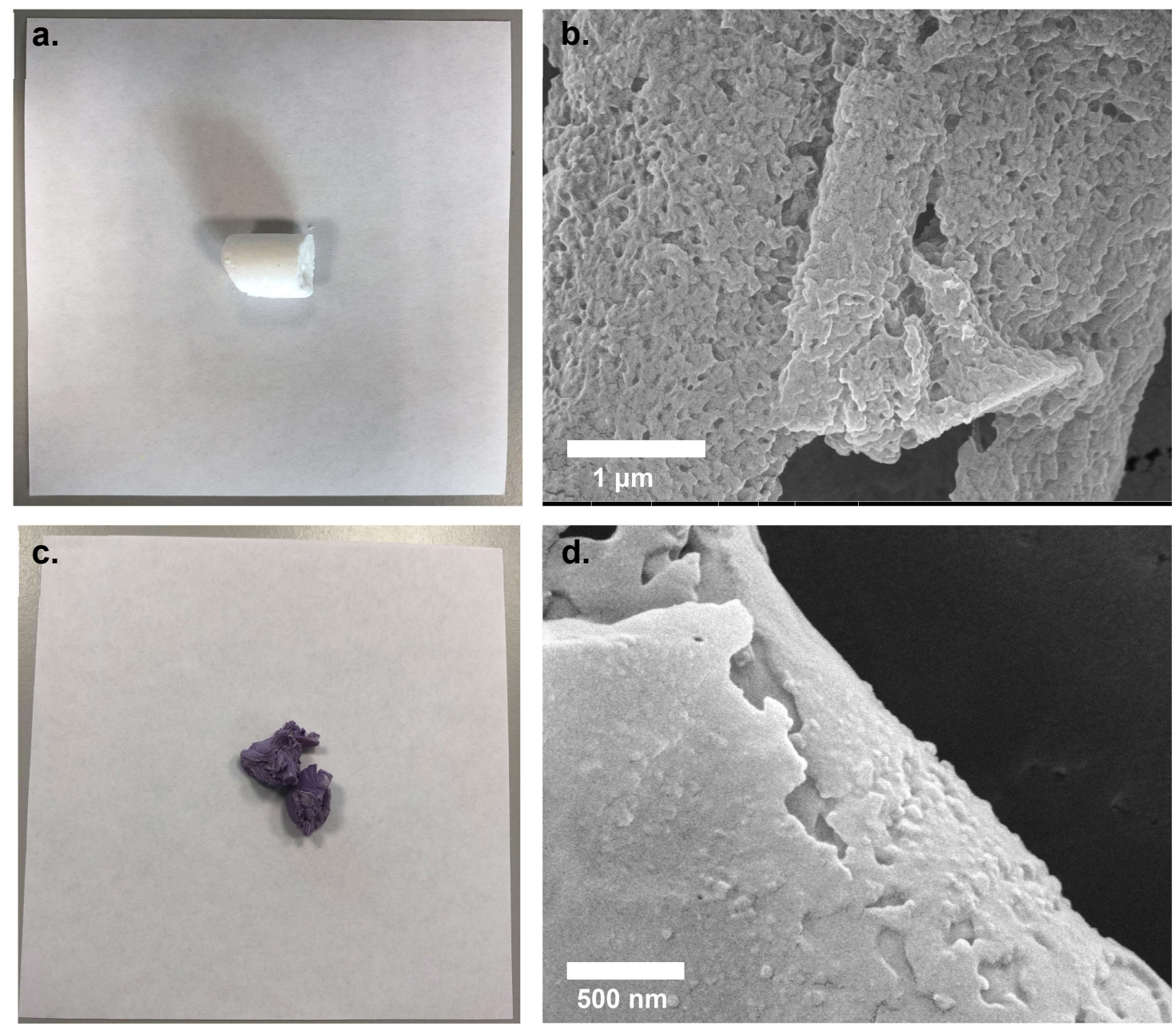

Figure S12. Morphological characterization. a. and c. Optical images, b. and d. SEM images of MOF808@PEG-PO3 and DOX@808@PEG-PO3. 

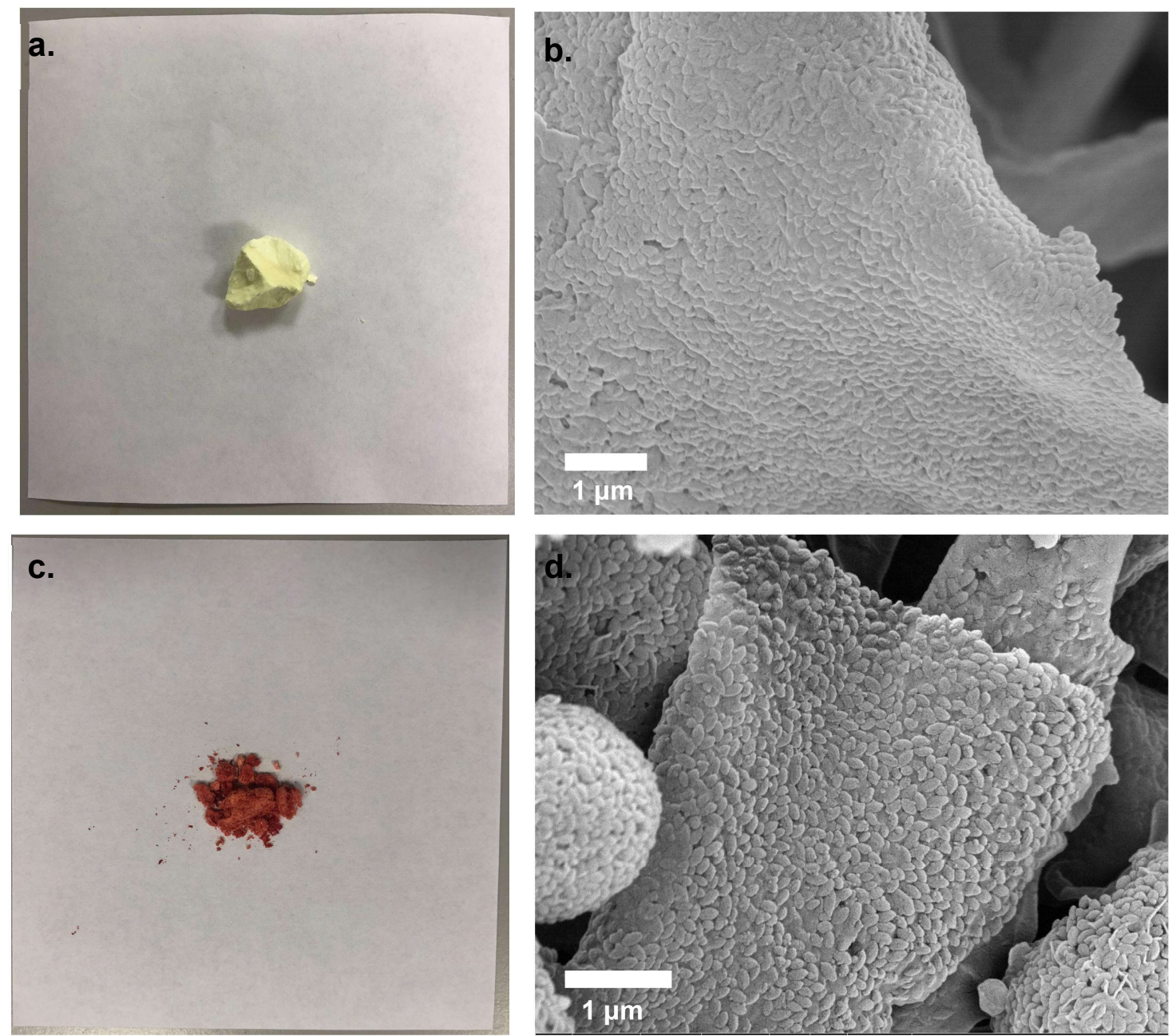

Figure S13. Morphological characterization. a. and c. Optical images, b. and d. SEM images of NU901@PEG-PO3 and DOX@901@PEG-PO3. 

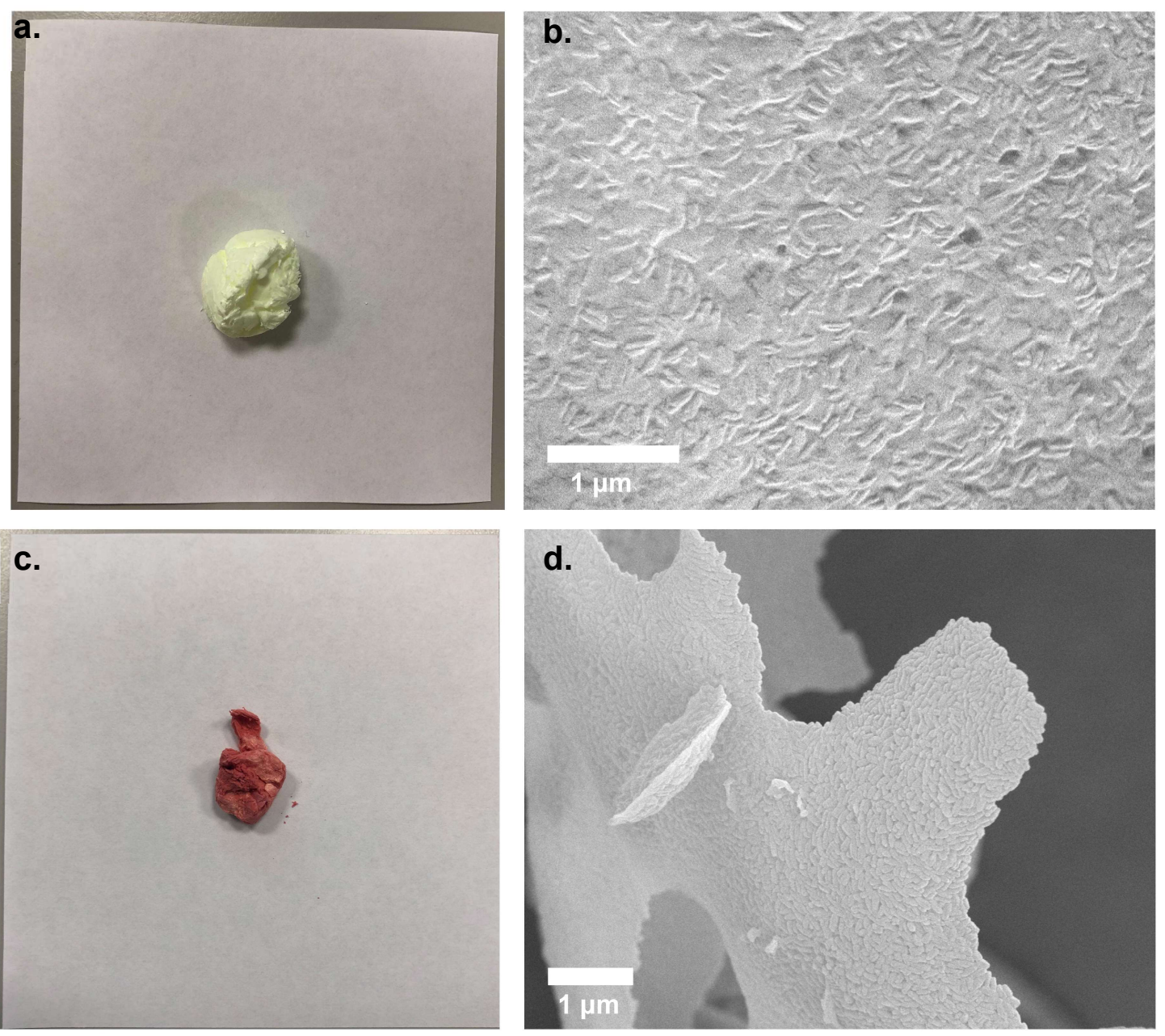

Figure S14. Morphological characterization. a. and c. Optical images, b. and d. SEM images of PCN128@PEG-PO3 and DOX@128@PEG-PO3.
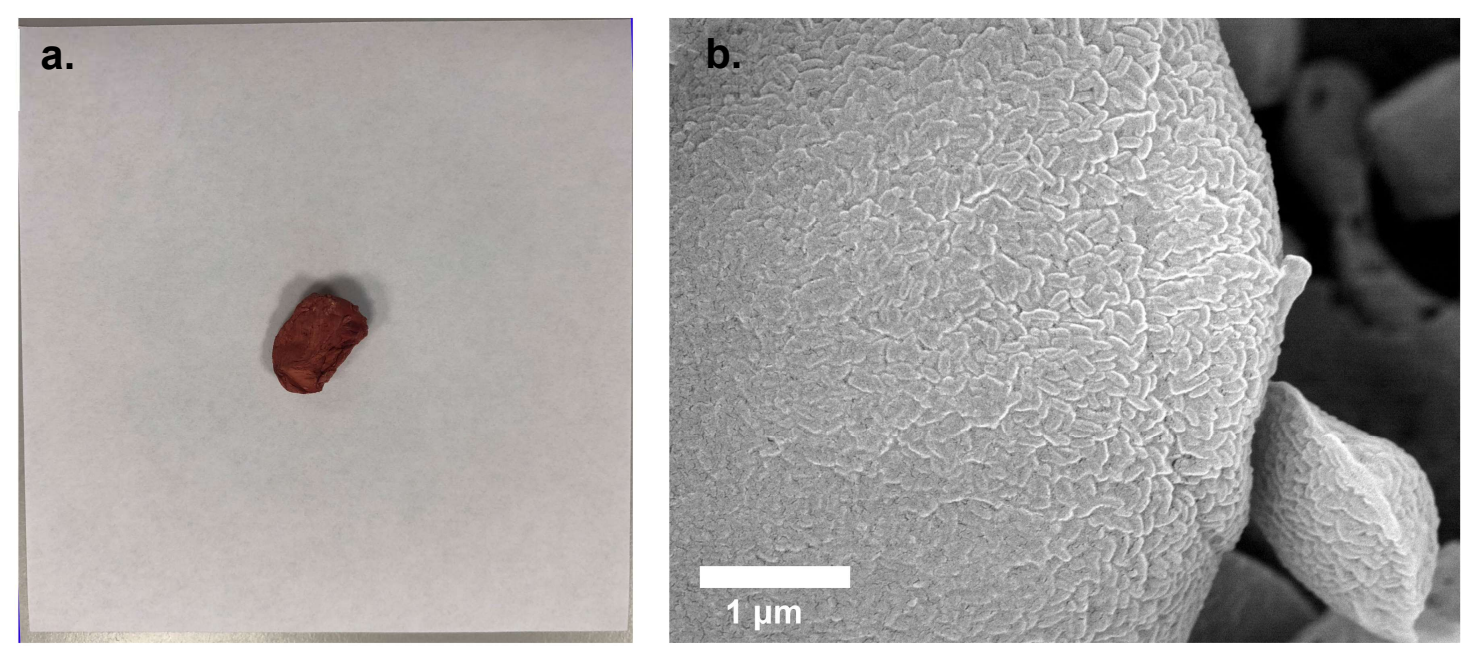

Figure S15. Morphological characterization. a. Optical image, b. SEM image of DOX@222@PEG-PO3. 

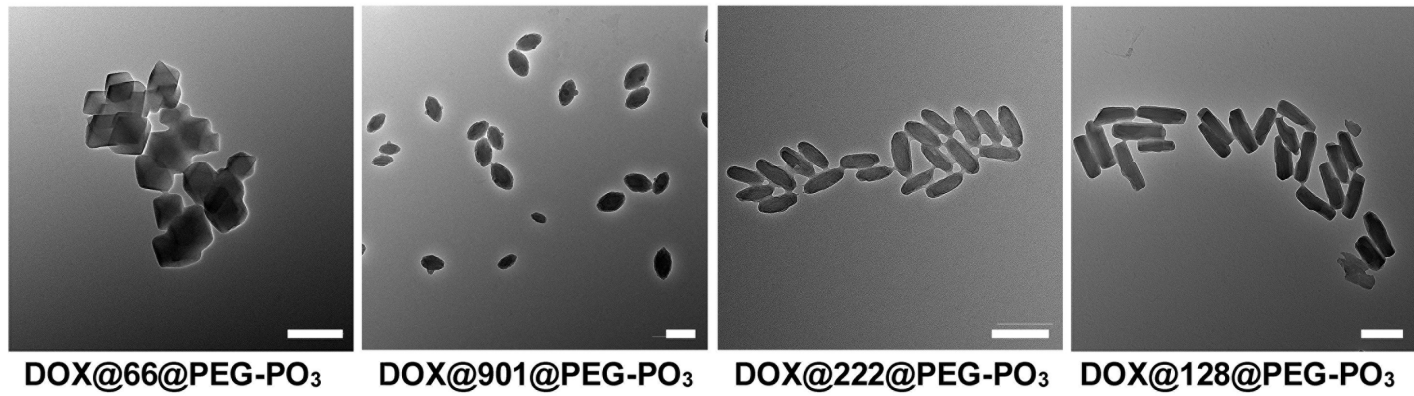

DOX@901@PEG-PO

DOX@222@PEG-PO3

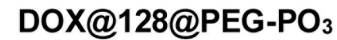

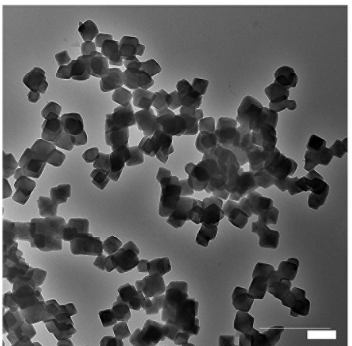

DOX@UiO-66

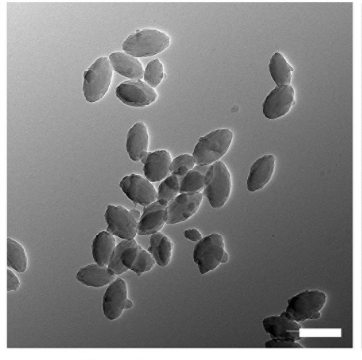

DOX@NU-901

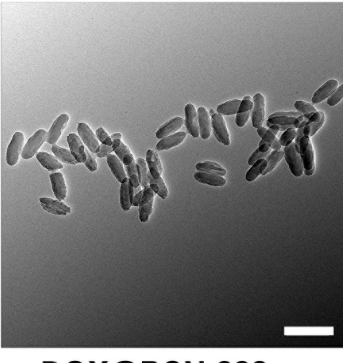

DOX@PCN-222

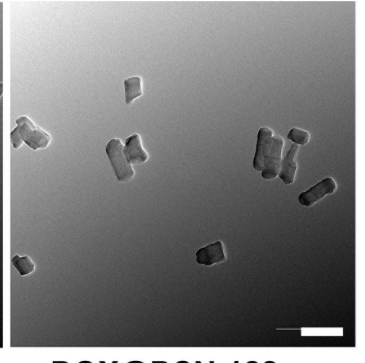

DOX@PCN-128

Figure S16. TEM images of DOX-loaded bare and PEGylated MOFs in water $(\mathrm{pH}=7.4)$ after 14 days. Scale bar: $200 \mathrm{~nm}$.
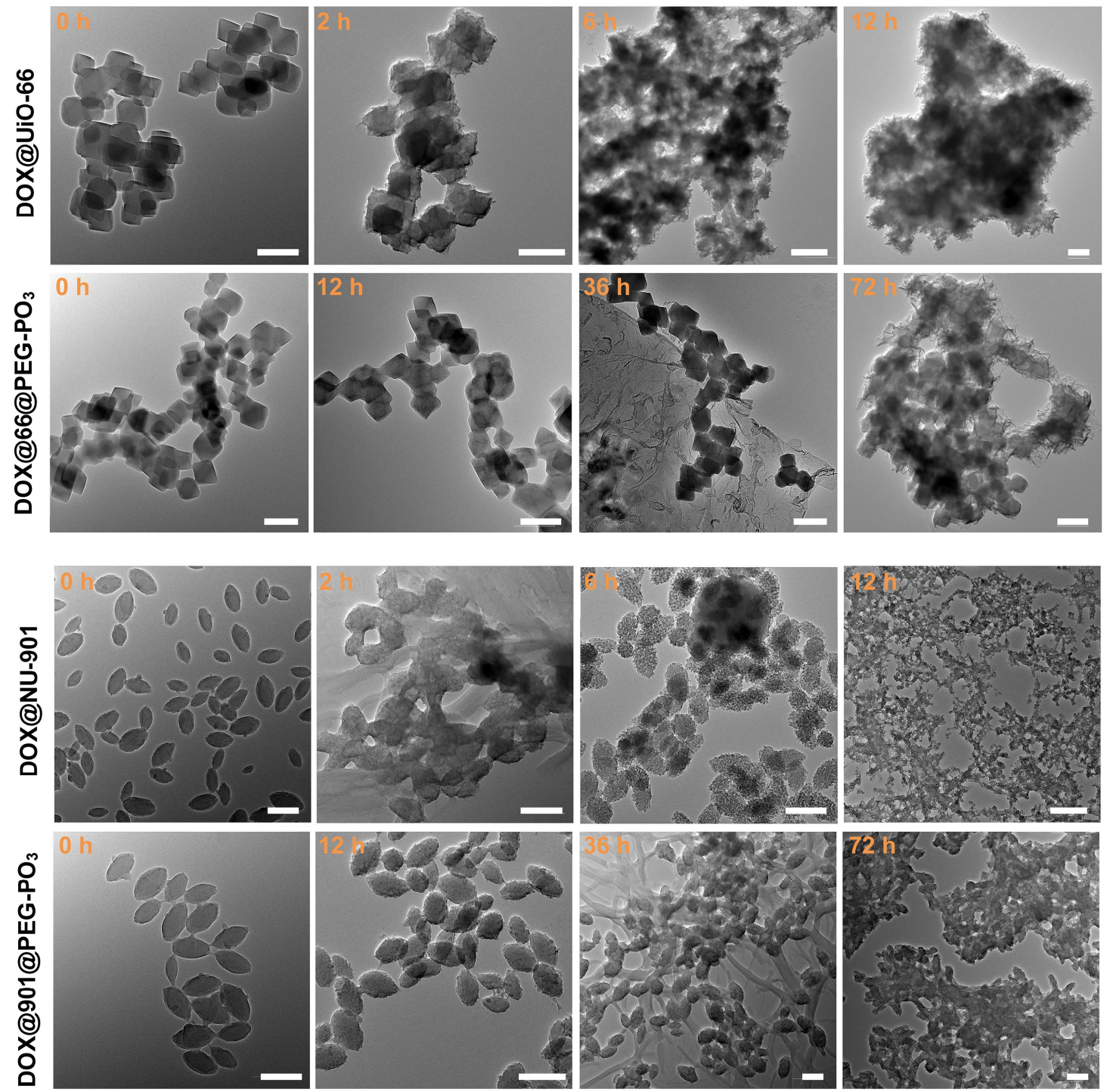

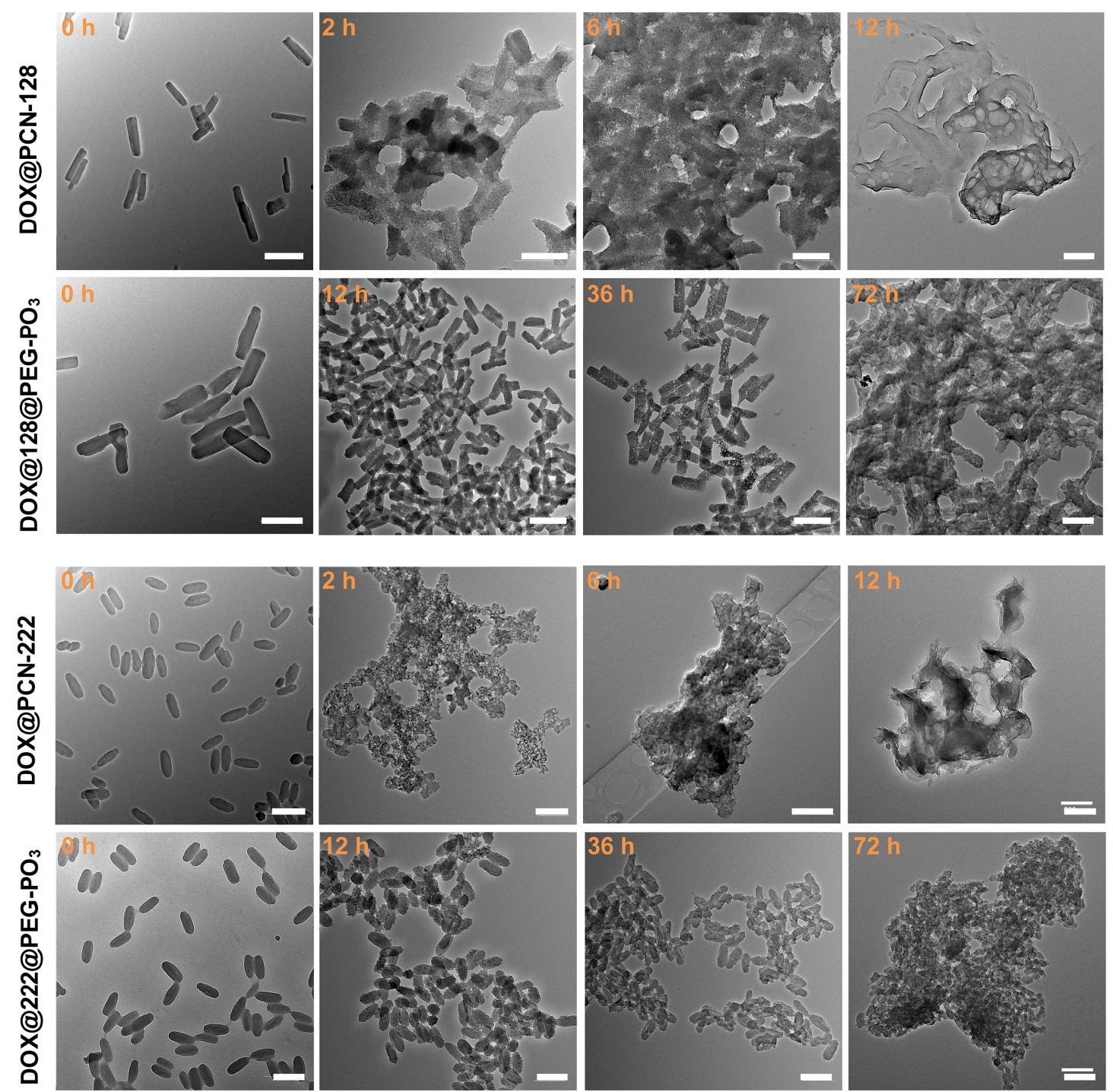

Figure S17. Time-dependent stability of DOX-loaded bare and PEGylated MOFs in PBS $(\mathrm{pH}=7.4)$ monitored by TEM. Scale bar: $200 \mathrm{~nm}$.
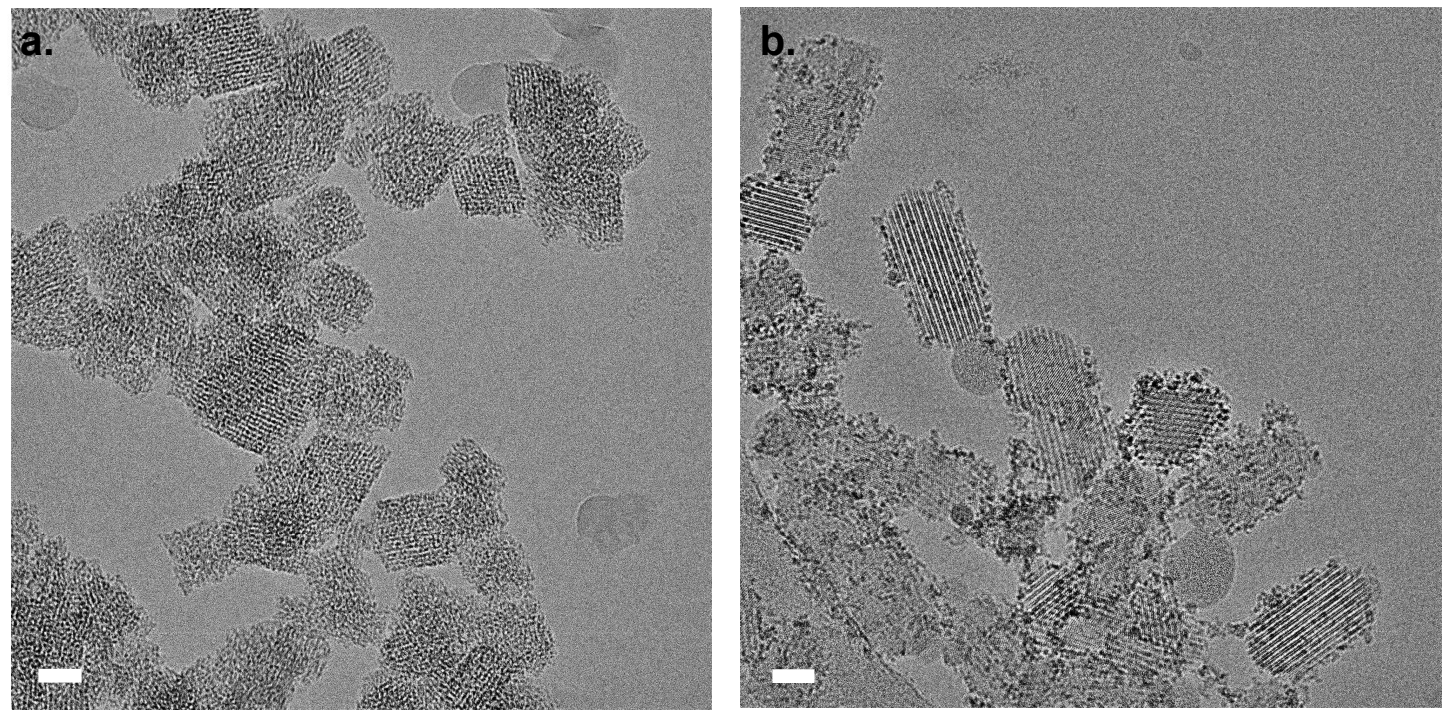

Figure S18. The Cryo-EM images of a. UiO-66@PEG- $\mathrm{PO}_{3}$ and b. $\mathrm{PCN}-222 @ \mathrm{PEG}-\mathrm{PO}_{3}$ after treating with PBS ( $\mathrm{pH}=7.4)$ for $24 \mathrm{~h}$. Scale bar: $50 \mathrm{~nm}$. 


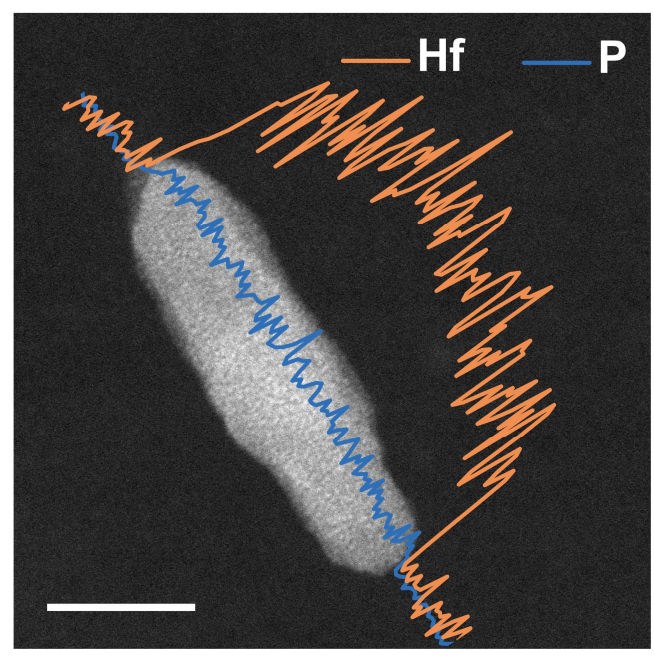

Figure S19. STEM image and EDX spectra of PCN-222@PEG-PO 3 after PEGylation of $16 \mathrm{~h}$. EDX line scans for $\mathrm{Hf}$ and $\mathrm{P}$ are in brown and blue, respectively. Scale bar, $50 \mathrm{~nm}$.

\section{XPS spectra}

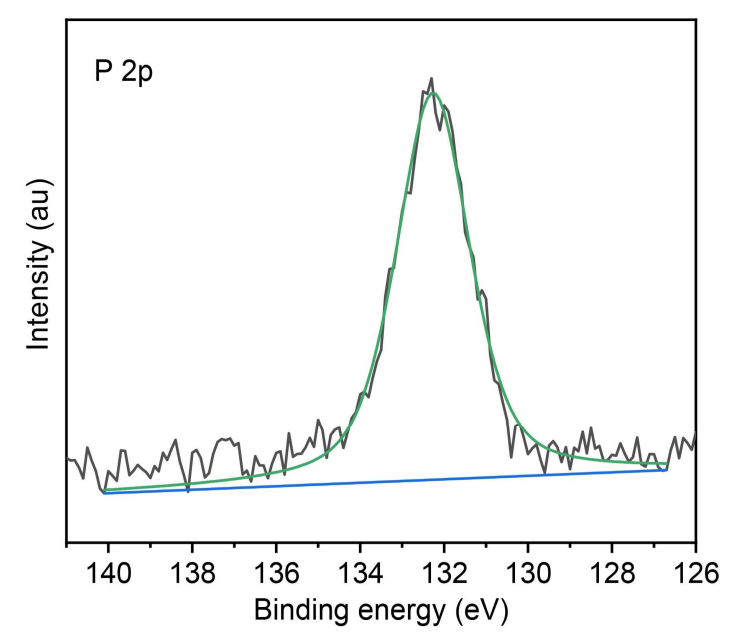

Figure S20. P 2p XPS spectrum of PCN-222@PEG-PO

a.

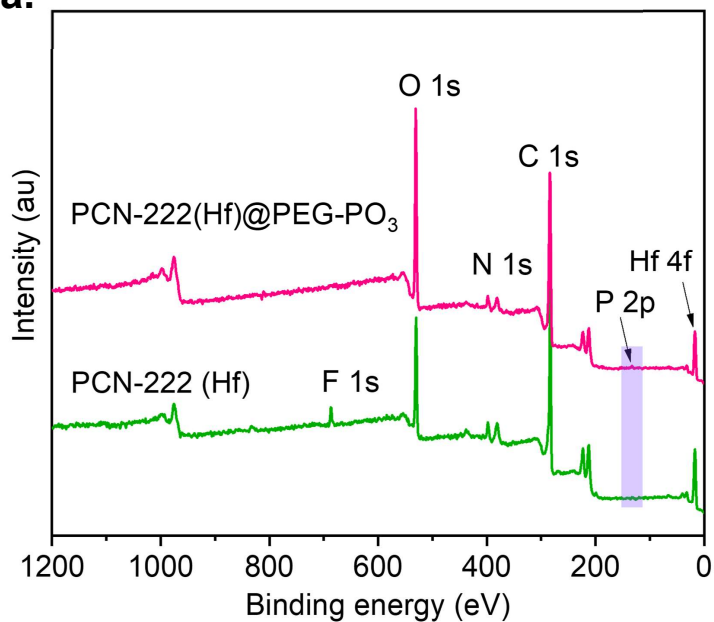

b.

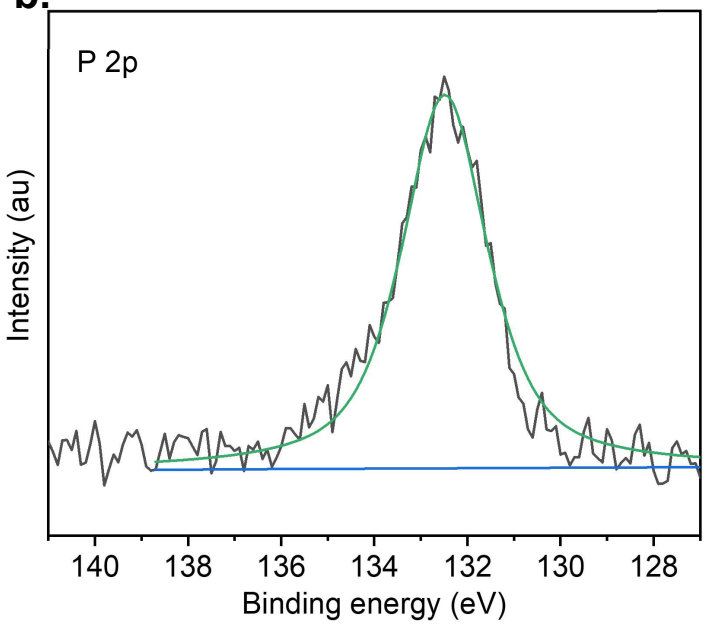

Figure S21. XPS spectra of PCN-222 (Hf) and PCN-222(Hf)@PEG-PO 3. a. XPS survey spectrum and b. highresolution of $\mathrm{P} 2 \mathrm{p}$ spectrum of $\mathrm{PCN}-222(\mathrm{Hf}) @ P E G-\mathrm{PO}_{3}$. 


\section{Photographs of the suspension and the related Tyndall effects}

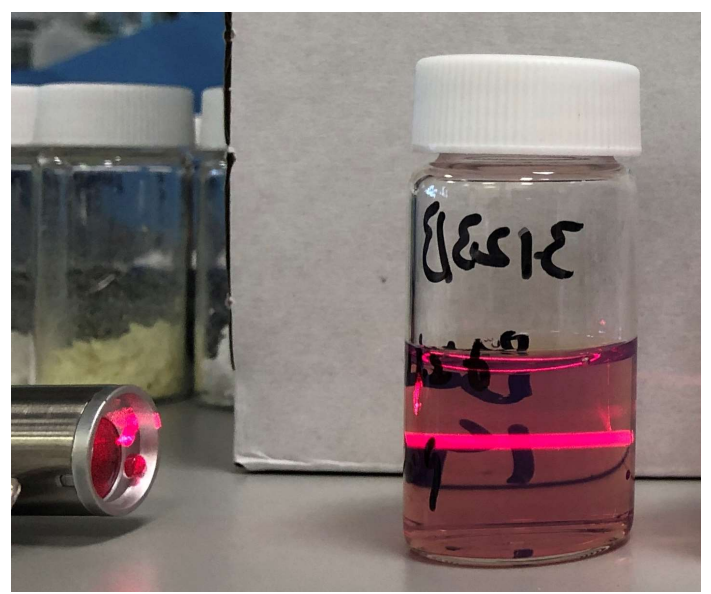

Figure S22. Typical Tyndall effect of PCN-222@PEG-PO

a.
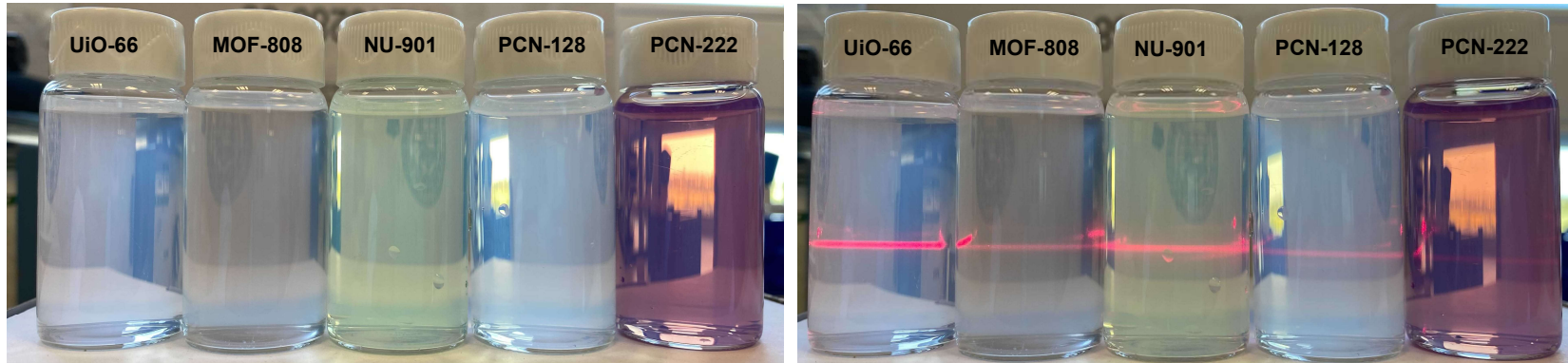

b.
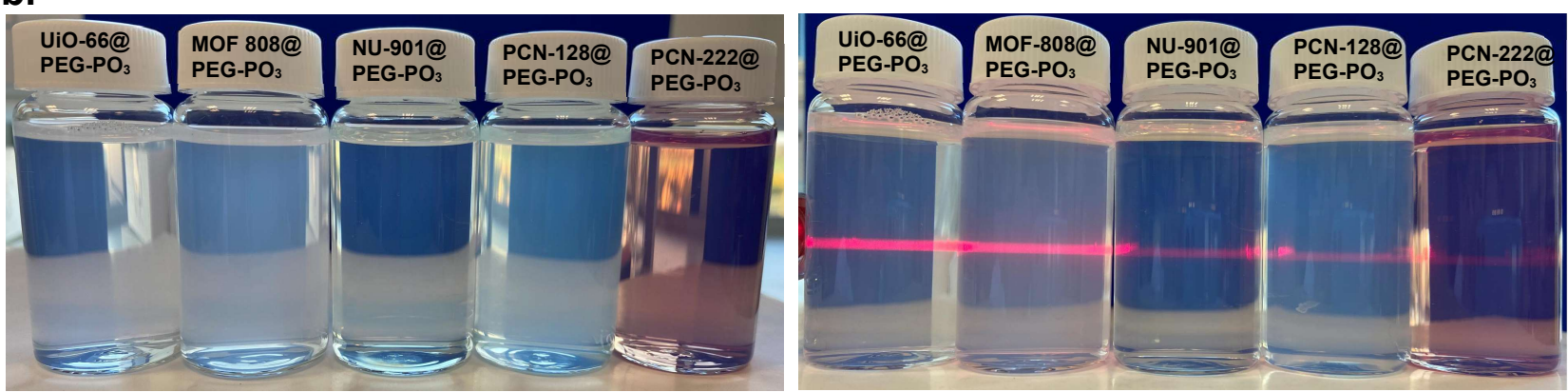

c.
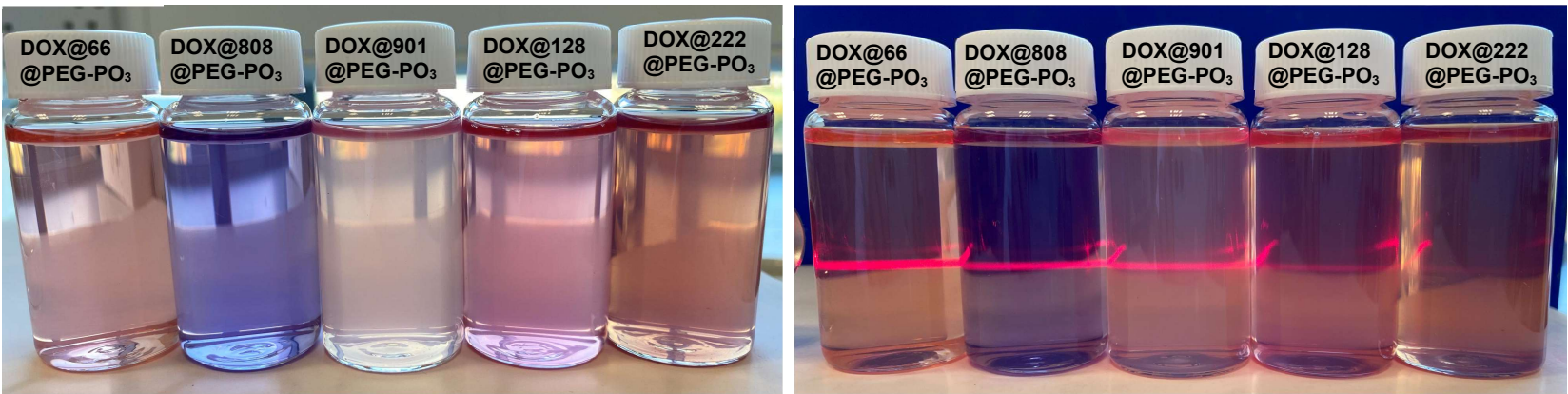

Figure S23. Optical images of the suspension and the related Tyndall effects at nanoMOFs concentration of $0.5 \mathrm{mg} / \mathrm{mL}$. a. Bare nanoMOFs, b, and c, the redispersed MOF@PEG-PO 3 and DOX@ MOF@PEG-PO ${ }_{3}$.The purple suspension was obtained for DOX@808@PEG-PO ${ }_{3}$, this is due to the complexation between DOX and $\mathrm{Zr}^{4+}$ that released from MOF-808. ${ }^{17}$ The UV-Vis spectra are shown in Figure $\mathbf{S 3 0 .}$ 


\section{PXRD patterns}
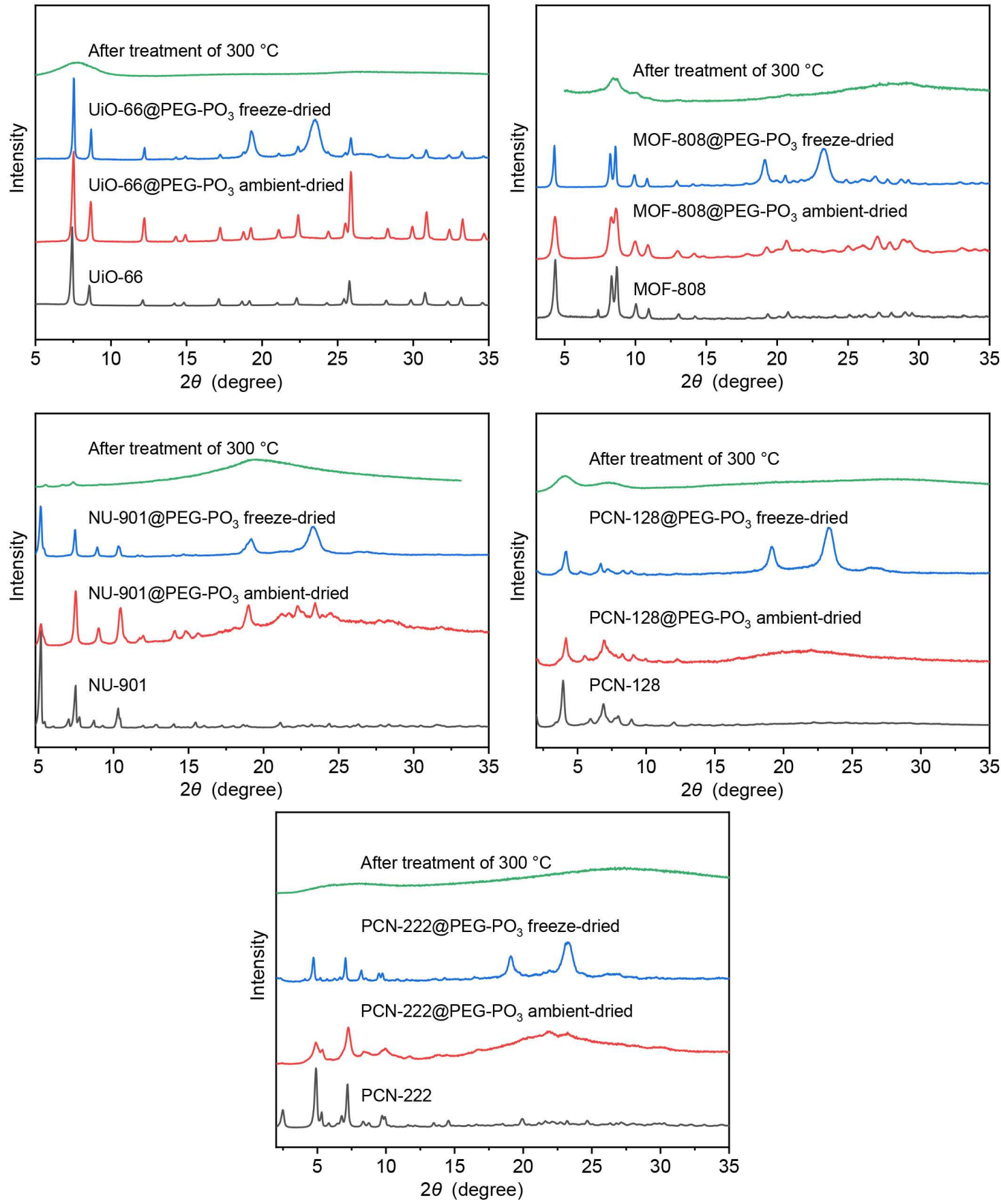

Figure S24. PXRD patterns of bare nanoMOFs, ambient-dried and lyophilized MOF@PEG-PO 3 , and MOF@PEG-PO 3 after heating under $300^{\circ} \mathrm{C}$ for $6 \mathrm{~h}$. 

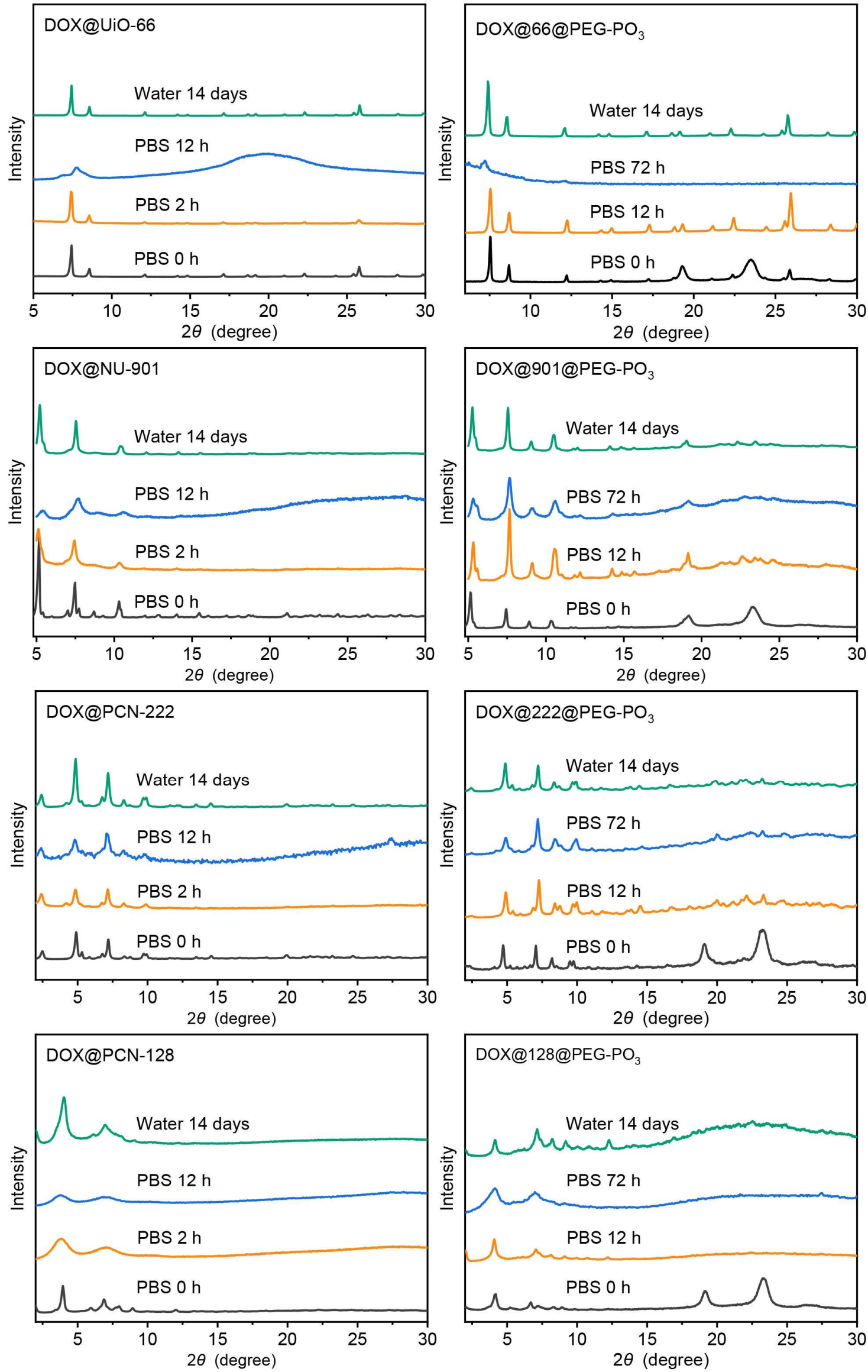

Figure S25. Time-dependent stability in PBS $(\mathrm{pH}=7.4)$ or water monitored by PXRD. 
a.

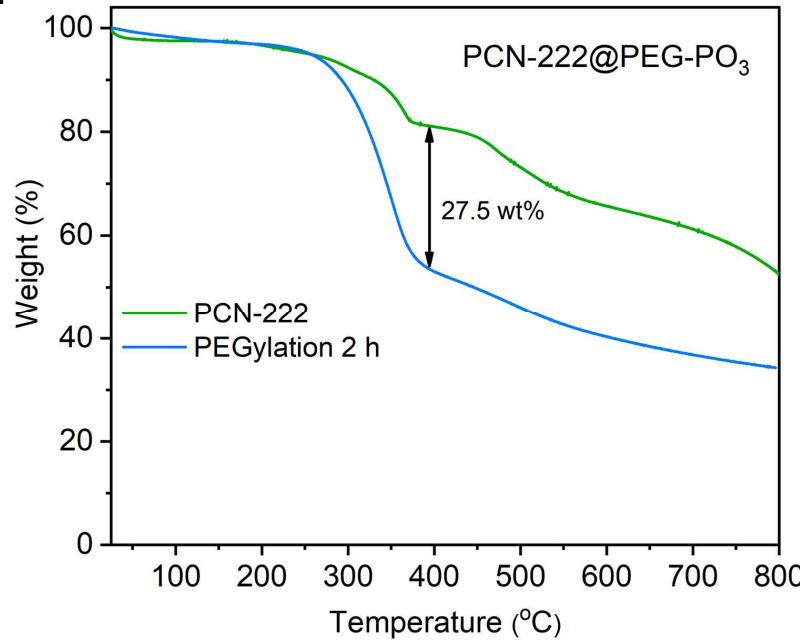

C.

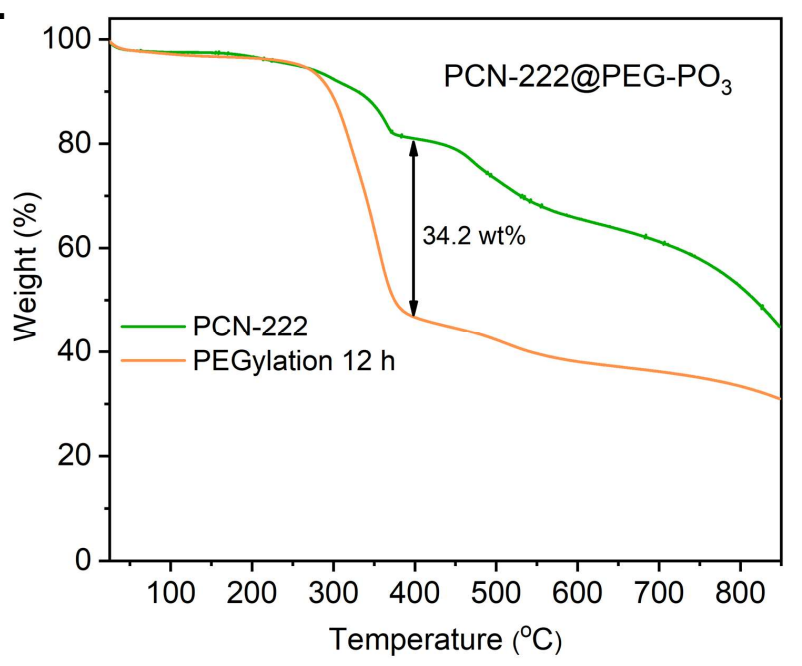

b.

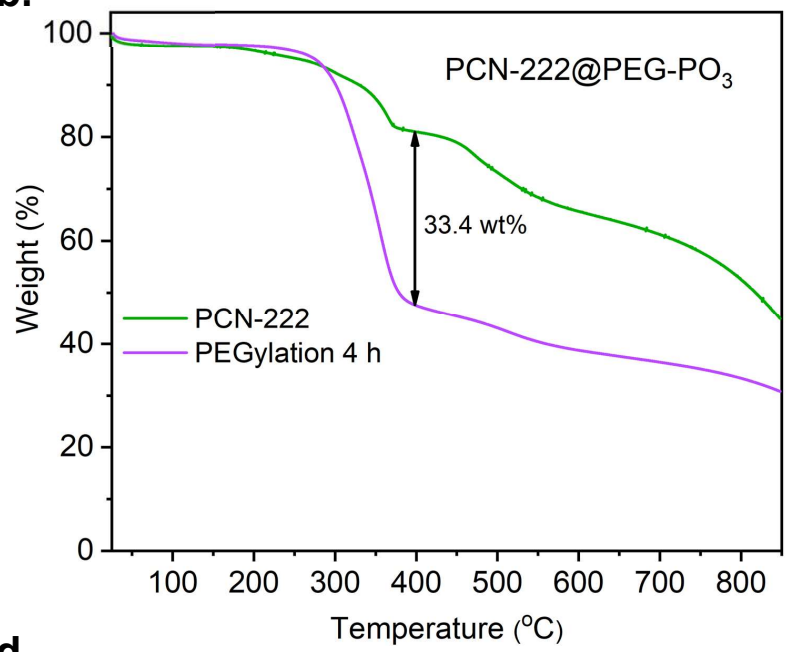

d.

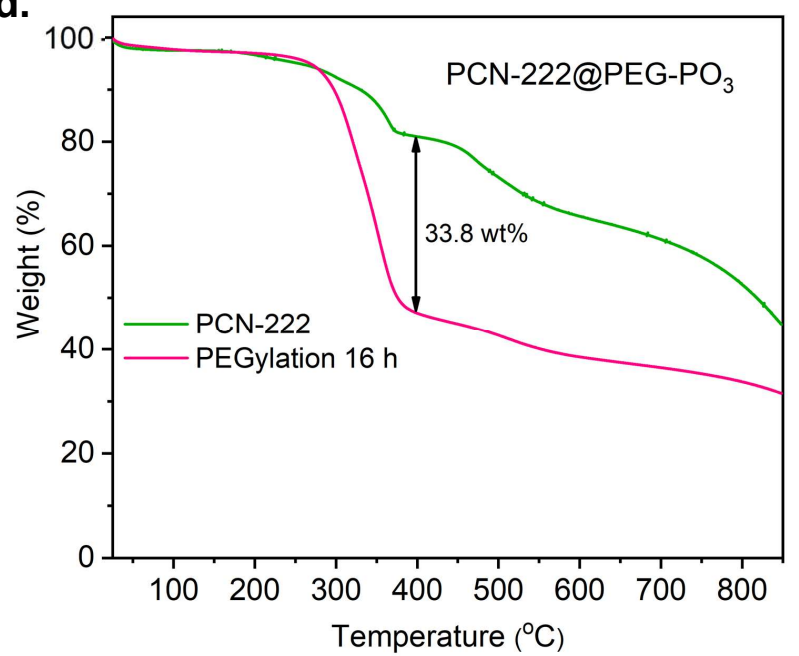

Figure S26. TGA profiles of PCN-222@PEG-PO and $\mathbf{d}$. PEGylation $16 \mathrm{~h}$. The encapsulated amount of $\mathrm{mPEG}-\mathrm{PO}_{3}$ was calculated based on the difference between the TGA curve of the first plateau $\left(376-452{ }^{\circ} \mathrm{C}\right) .{ }^{18-19}$ 


\section{Additional figure}

a.

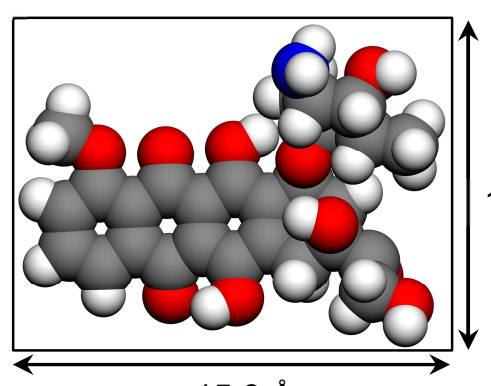

$15.8 \AA$

b.

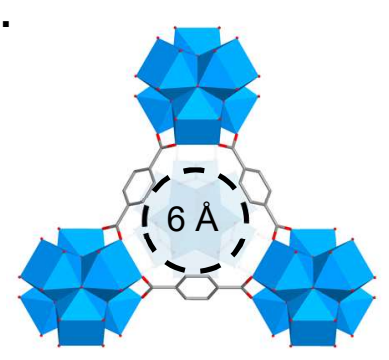

UiO-66

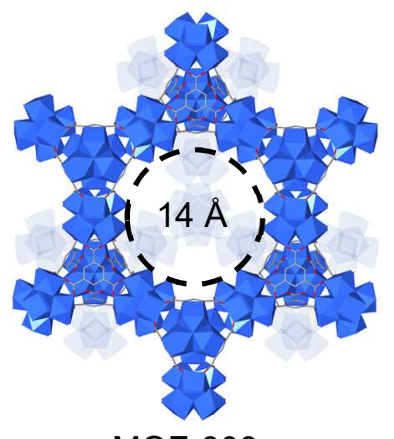

MOF-808

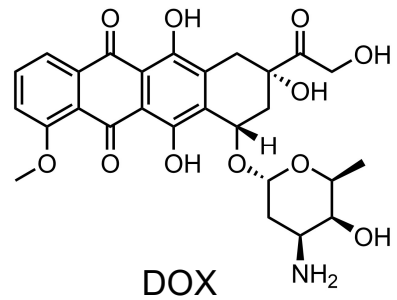

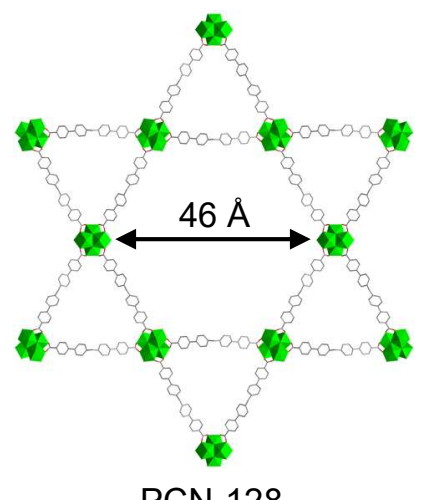

$\mathrm{PCN}-128$

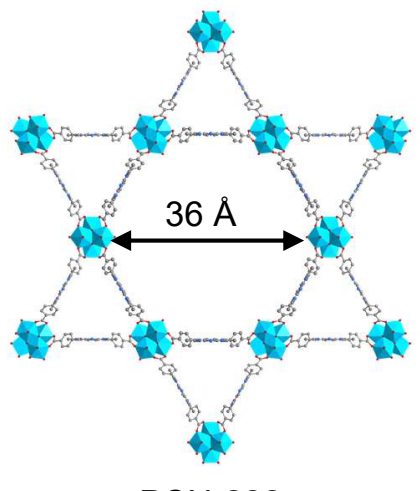

$\mathrm{PCN}-222$

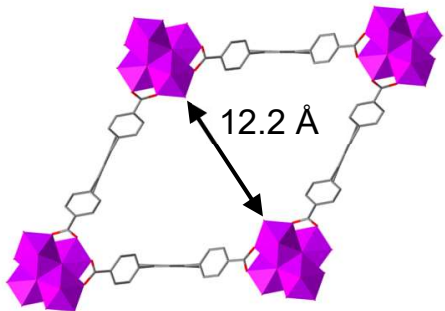

NU-901

Figure S27. a. Calculated molecular model and molecular structures of DOX. (The space-filling models were calculated by Materials Studio). b. Illustration of the pore size of UiO-66, MOF-808, NU-901, PCN-128 and PCN-222. 


\section{FT-IR spectra}
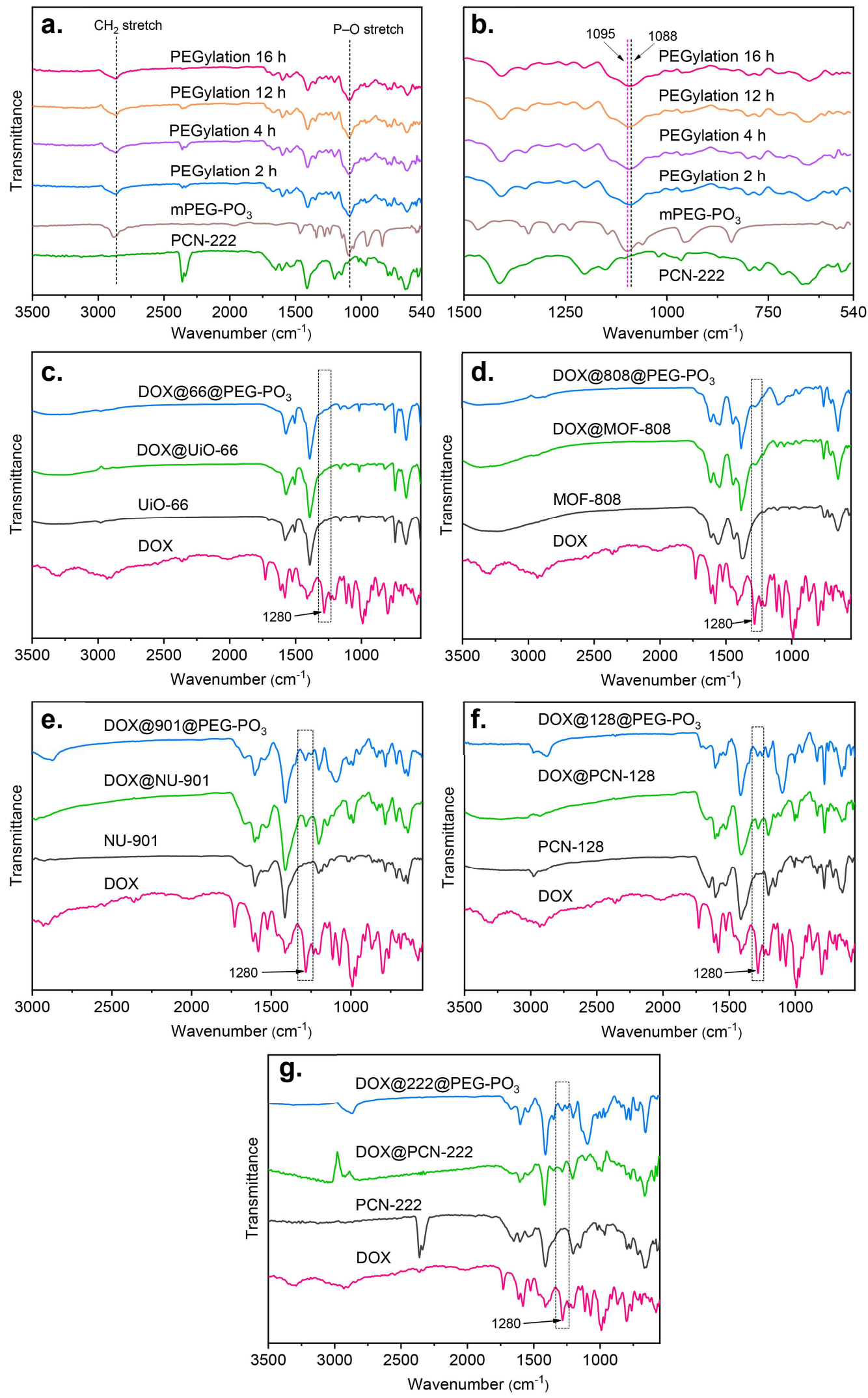

Figure S28. a. Full FT-IR spectra. b. The enlarged spectra of PEGylated PCN-222 at different time points. The appearance of two new bands at 2866 and $1090 \mathrm{~cm}^{-1}$ were attributed to the stretching vibration of $\mathrm{C}-\mathrm{H}$ and P-O from mPEG-PO ${ }_{3}{ }^{20}$ (c - g) FT-IR spectra of DOX, bare nanoMOFs, DOX@MOF, DOX@MOF@PEG$\mathrm{PO}_{3}:$ c. UiO-66, d. MOF-808, e. NU-901, f. PCN-128 and g. PCN-222. 
UV-Vis spectra
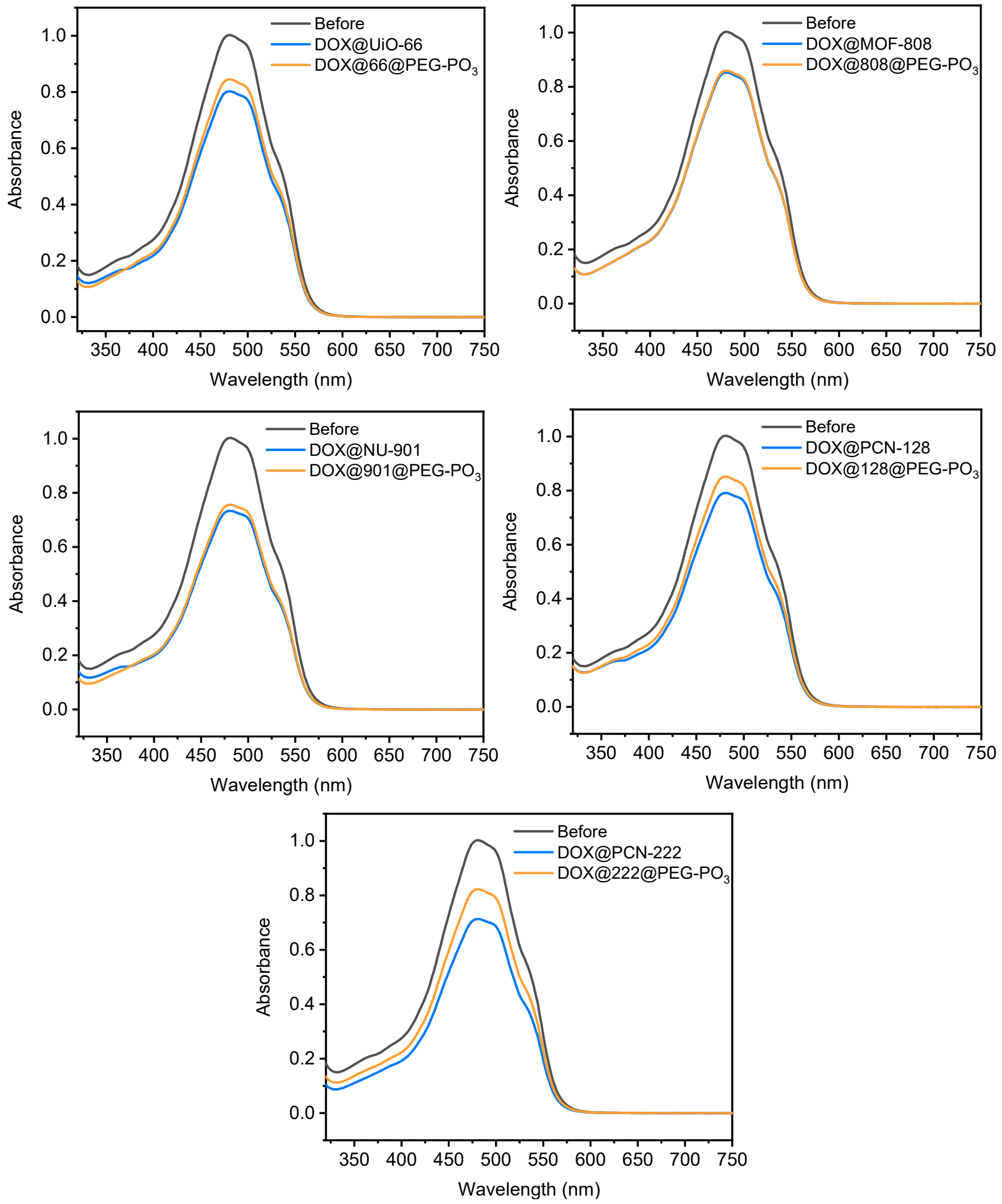

Figure S29. UV-Vis spectra of the pristine DOX solution, after drug loading and PEGylation. 

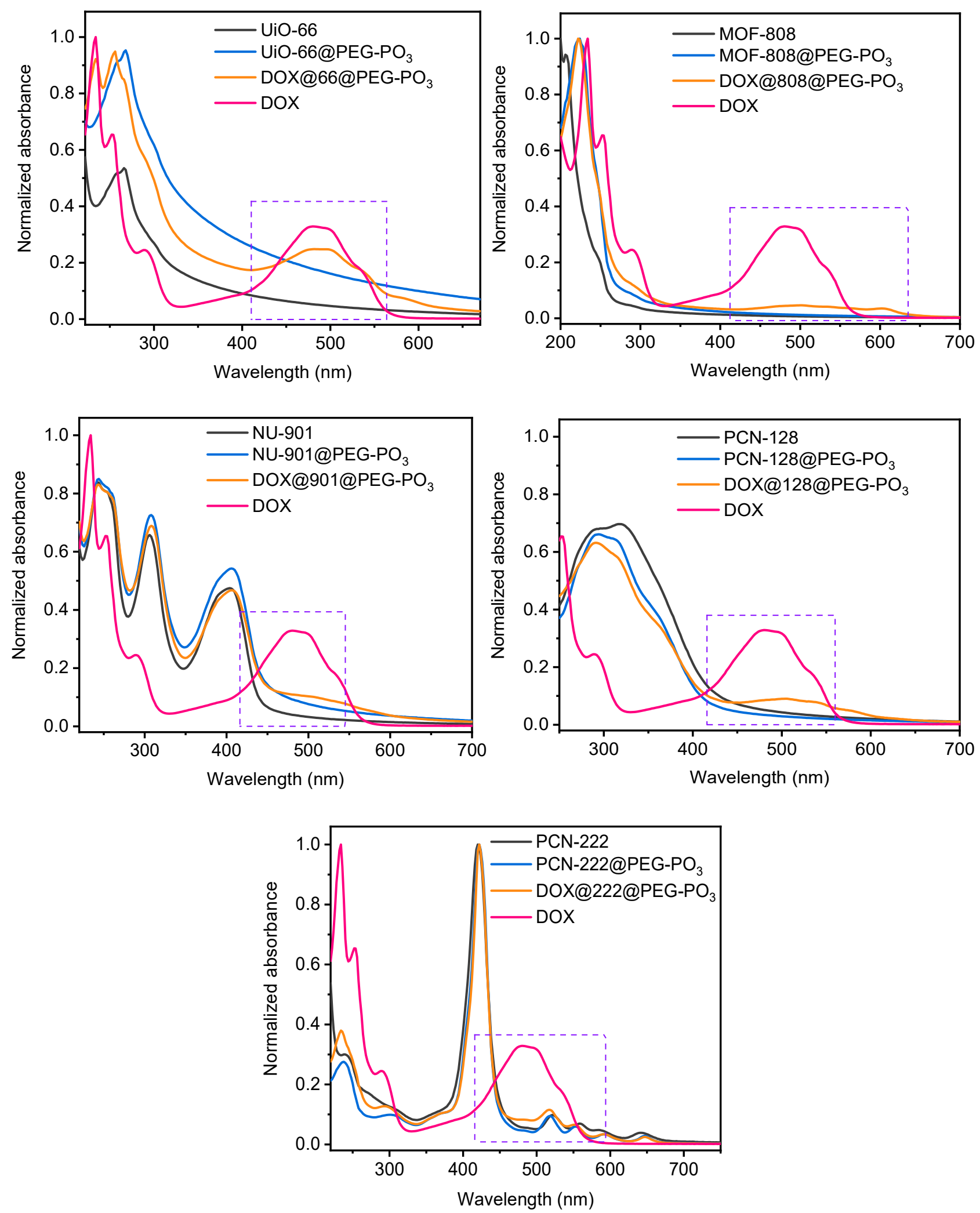

Figure S30. UV-Vis spectra of DOX, bare nanoMOFs, MOF@PEG-PO 3 and DOX@MOF@PEG-PO 3 .The purple boxes show the presence of DOX in PEGylated MOFs. It is worth recalling that the absorption spectrum of DOX displays bands at $288 \mathrm{~nm}$ and $480-500 \mathrm{~nm} .{ }^{21}$ We note that there is either a sharp or broad peak in related positions of DOX@MOF@PEG-PO3. Briefly, peaks ranged from 400 to 600 nm were observed for DOX@66@PEG-PO 3 , DOX@901@PEG-PO 3 and DOX@128@PEG-PO 3. In the case of DOX@808@PEG$\mathrm{PO}_{3}$, a broad $450 \mathrm{~nm}$ to $650 \mathrm{~nm}$ was observed. The slight shift of its absorbance of DOX-loaded is due to the complexation between DOX and $\mathrm{Zr}^{4+}$ that released from MOF-808. ${ }^{17}$ At the same time, the colour of its 
suspension has changed into purple (Figure S23c). As for the spectrum of DOX@222@PEG-PO absorption of DOX overlaps with that of PCN-222; that is the reason why the UV absorption peak of DOX in PEGylated PCN-222 is not obvious. In addition, a similar result has also been found in a previously reported paper. $^{22}$
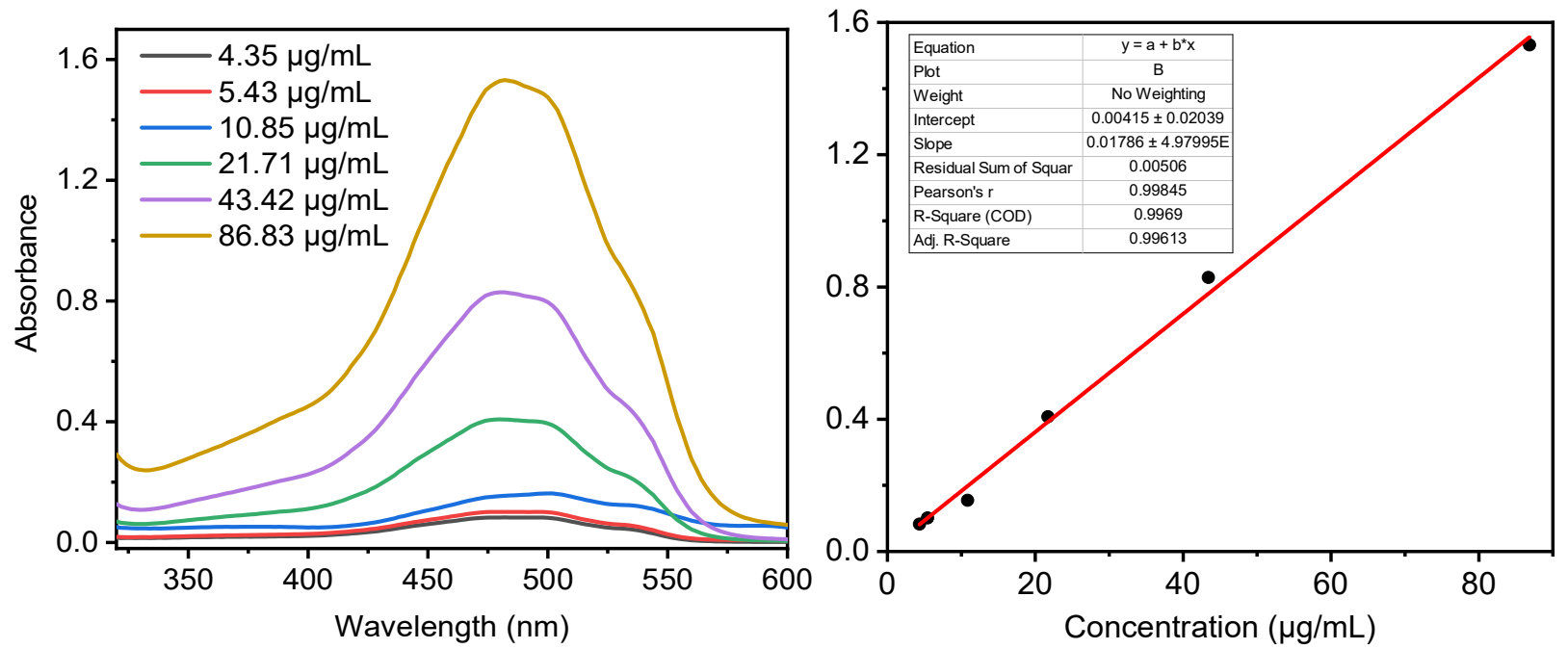

Figure S31. Calibration curve of DOX in $\mathrm{H}_{2} \mathrm{O}$ (maximum absorbance at $486 \mathrm{~nm}$ ).

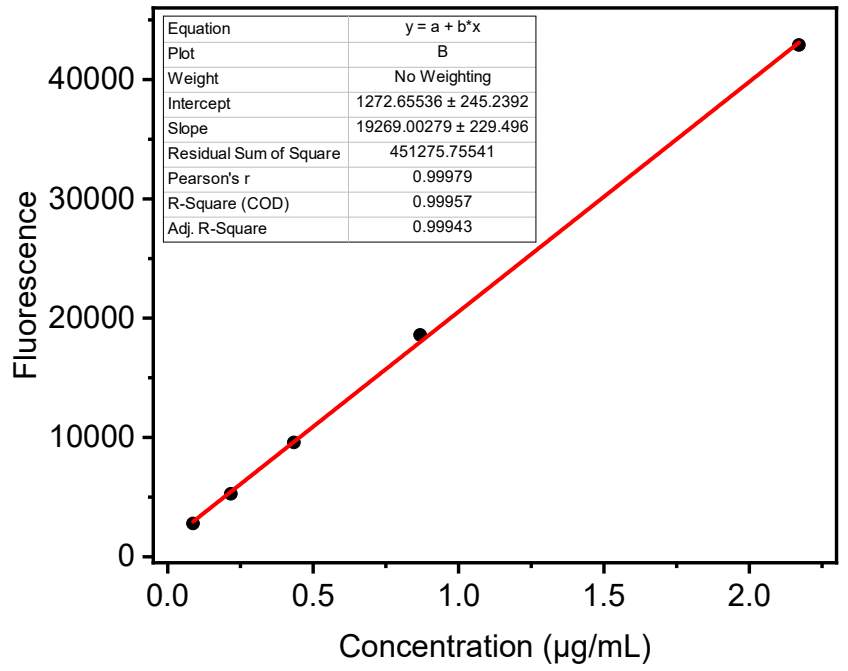

Figure S32. Calibration curve of DOX in PBS $(\mathrm{pH}=7.4)$ (emission maximum at $595 \mathrm{~nm}$ when excited at 486 $\mathrm{nm})$. 


\section{$\mathrm{N}_{2}$ sorption isotherms}

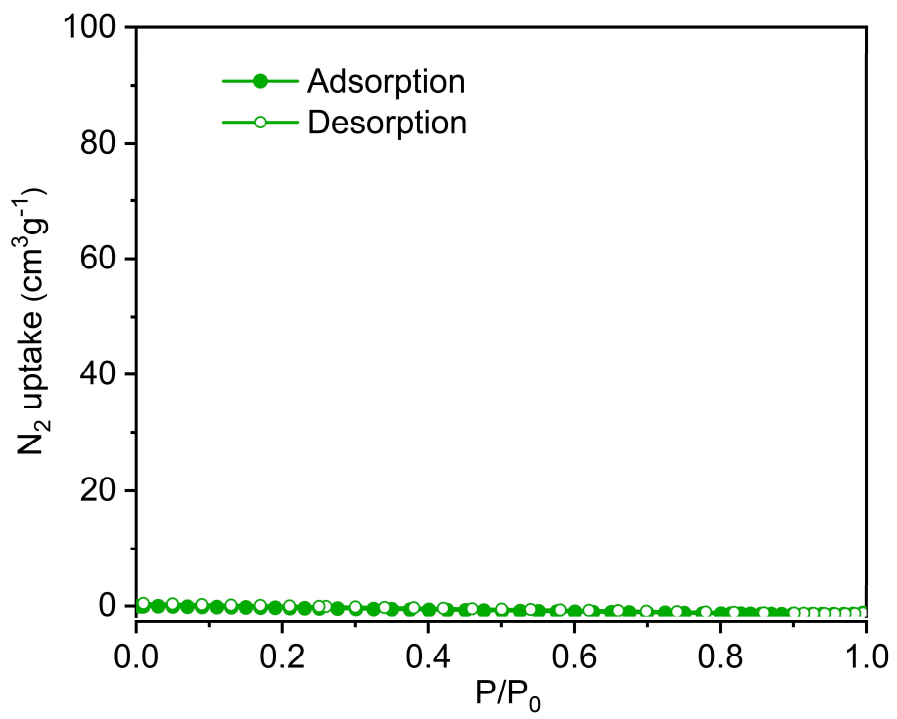

Figure S33. $\mathrm{N}_{2}$ adsorption isotherms of pure $\mathrm{mPEG}-\mathrm{PO}_{3}$.

a.
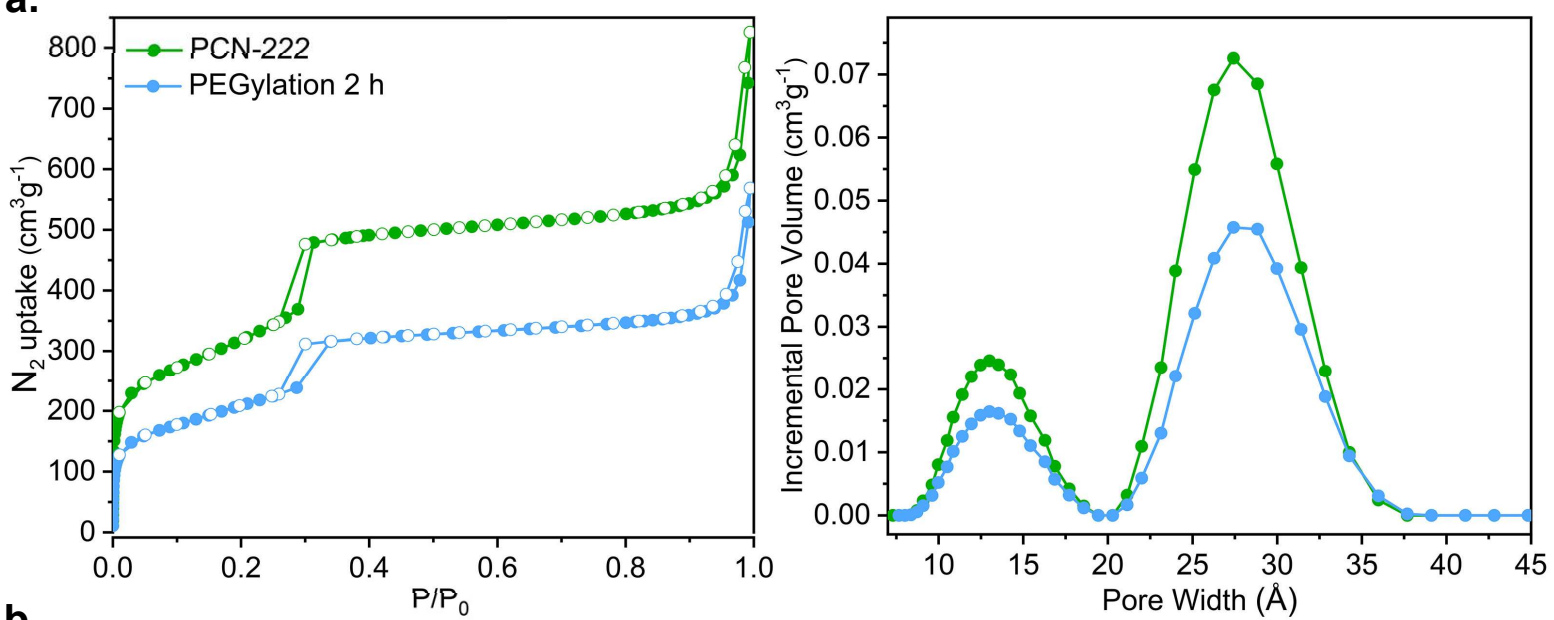

b.
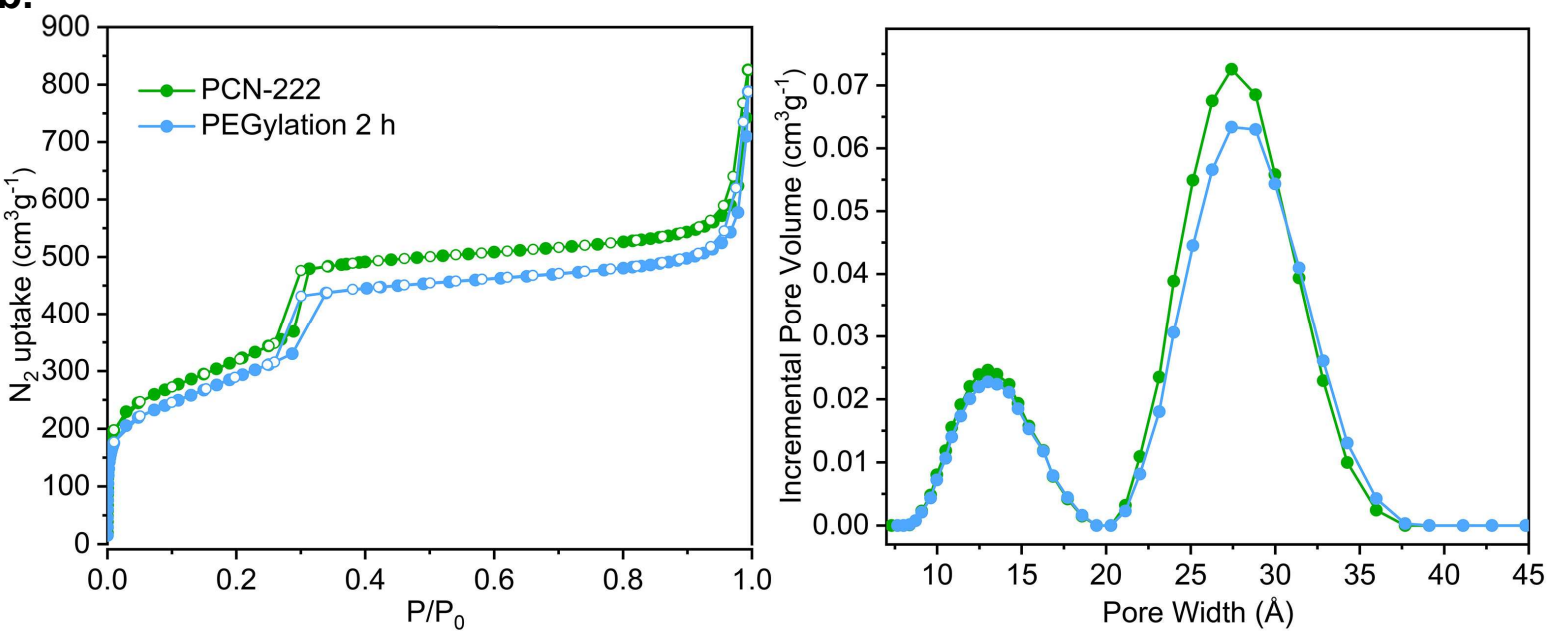

Figure S34. $\mathrm{N}_{2}$ adsorption isotherms and PSD of PCN-222 and PEGylation $2 \mathrm{~h}$. $\mathbf{a}$. before and $\mathbf{b}$. after subtracting the amount of $\mathrm{mPEG}-\mathrm{PO}_{3}$ incorporated. 
a.
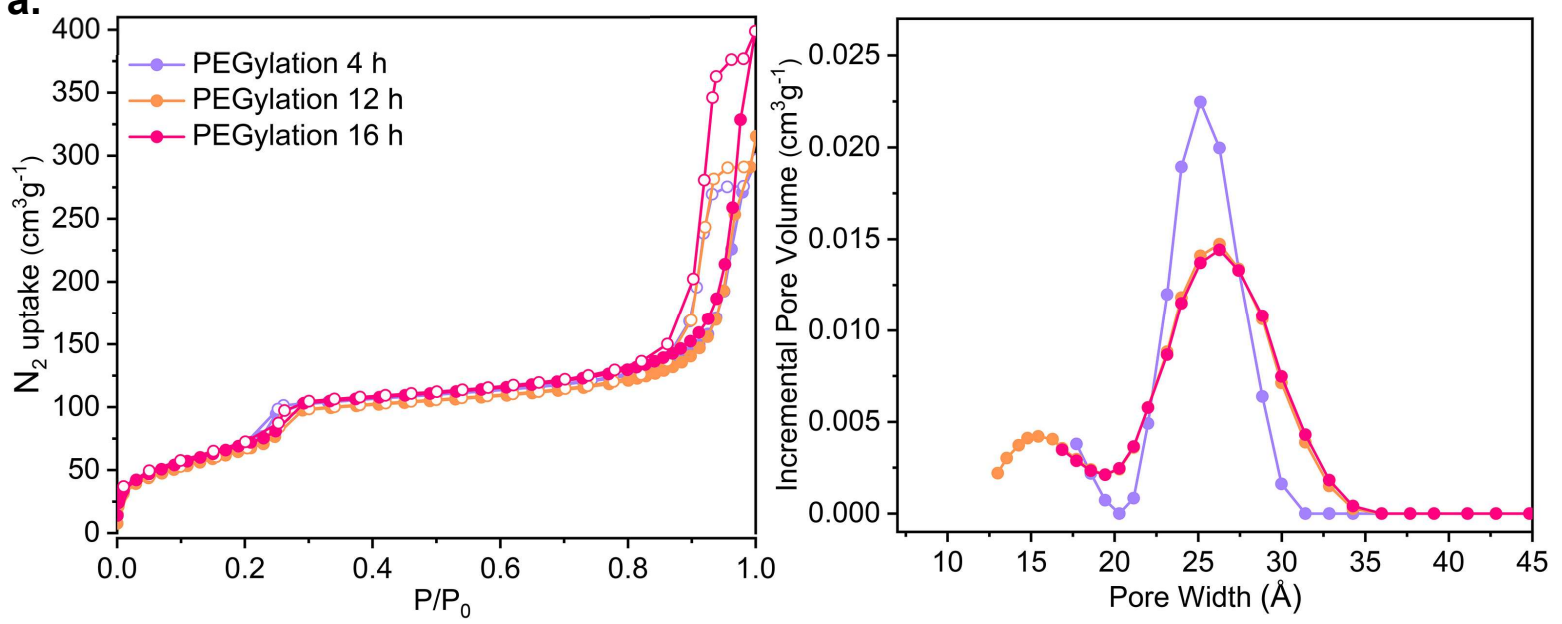

b.
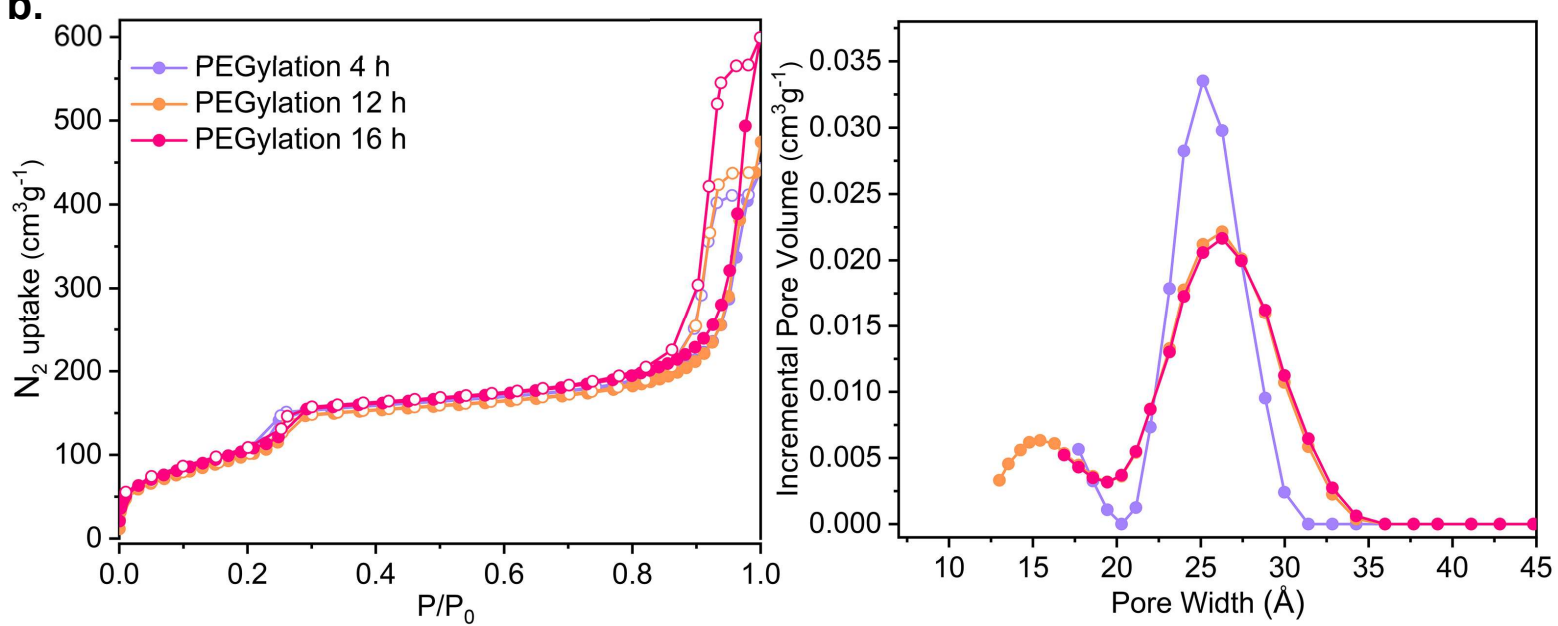

Figure S35. $\mathrm{N}_{2}$ adsorption isotherms and PSD of PEGylation 4, 12 and $16 \mathrm{~h}$. $\mathbf{a}$. before and $\mathbf{b}$. after subtracting the amount of $\mathrm{mPEG}-\mathrm{PO}_{3}$ included.
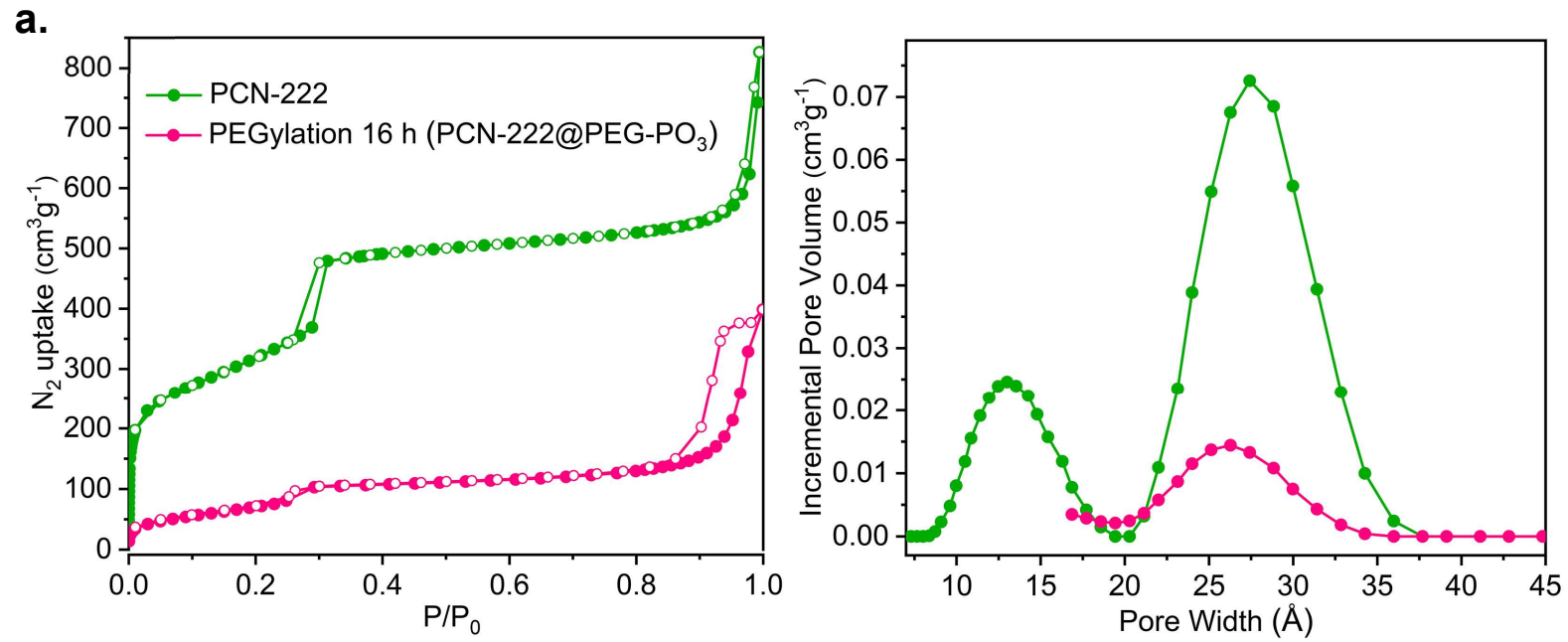
b.
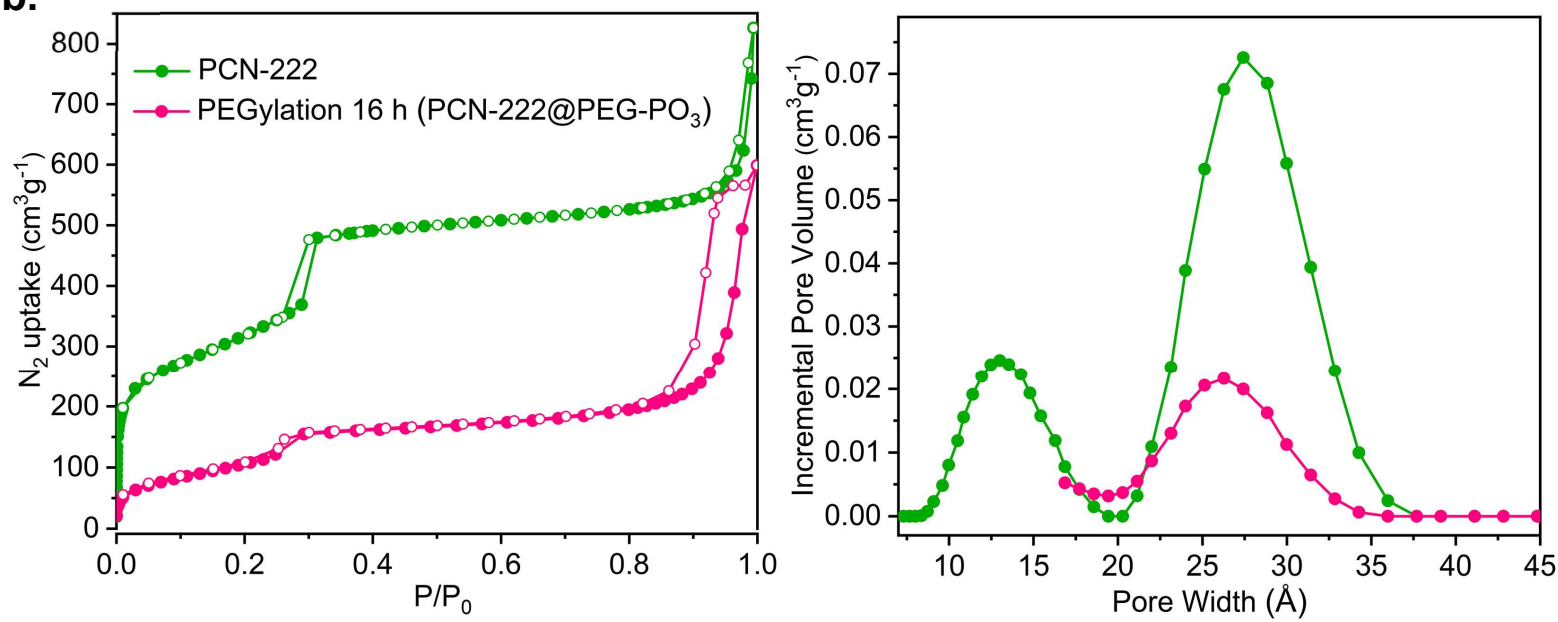

Figure S36. $\mathrm{N}_{2}$ adsorption isotherms and PSD of PCN-222 and PEGylation $16 \mathrm{~h}$. a. before and $\mathbf{b}$. after subtracting the amount of $\mathrm{mPEG}-\mathrm{PO}_{3}$ included.

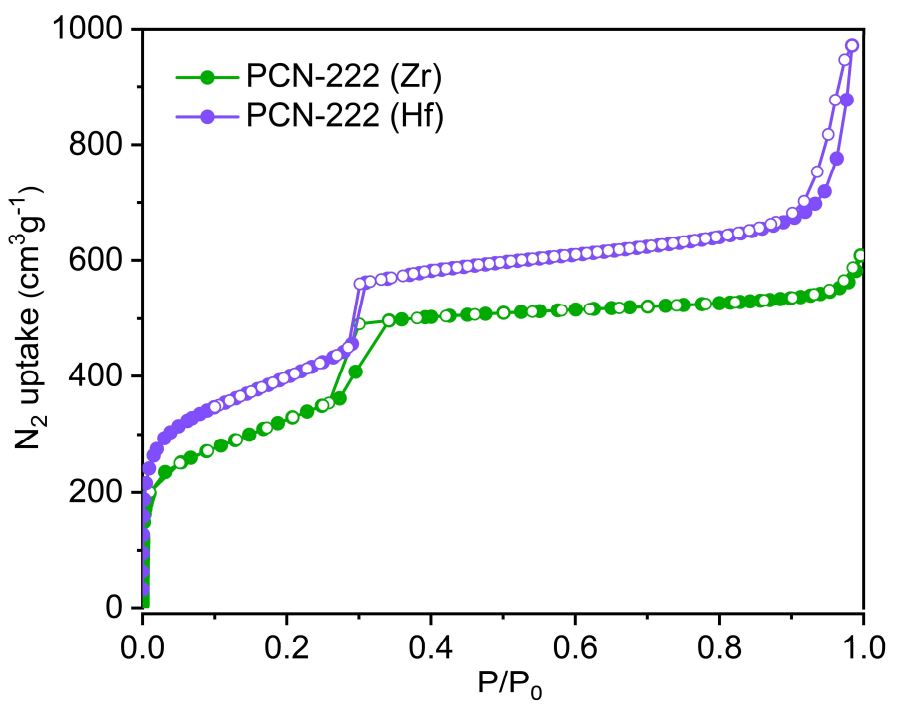

Figure S37. $\mathrm{N}_{2}$ adsorption isotherms of PCN-222 ( $\mathrm{Zr}$ and $\left.\mathrm{Hf}\right)$.

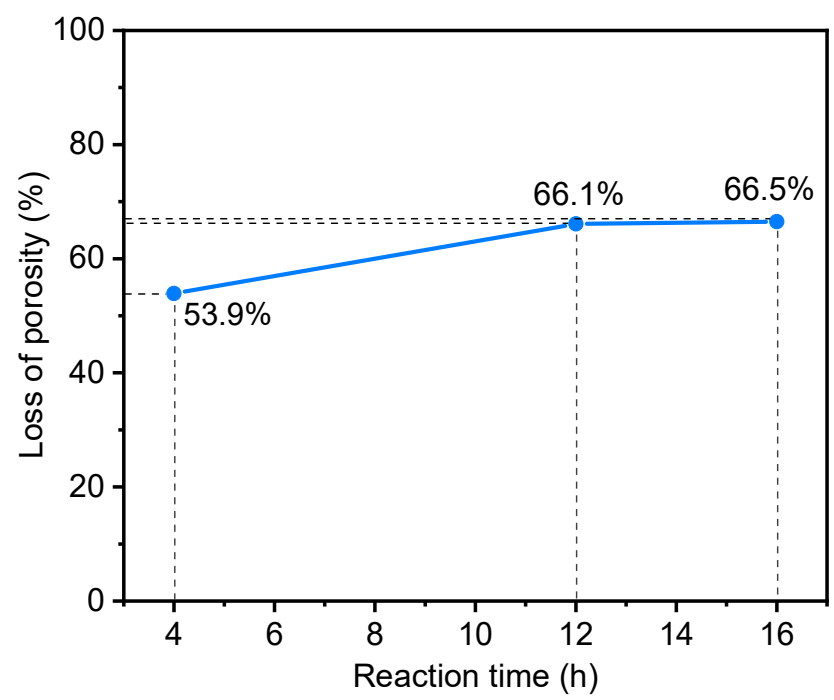

Figure S38. Correlation between loss of porosity and reaction time. 
a.

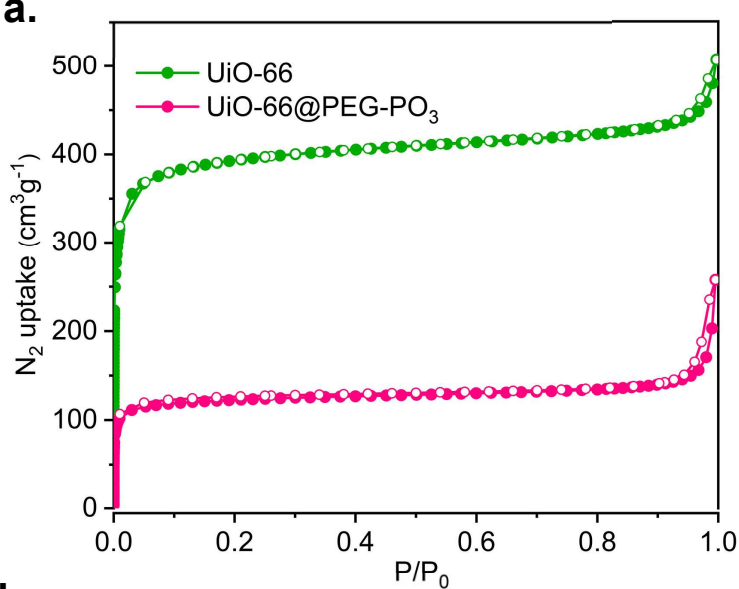

b.
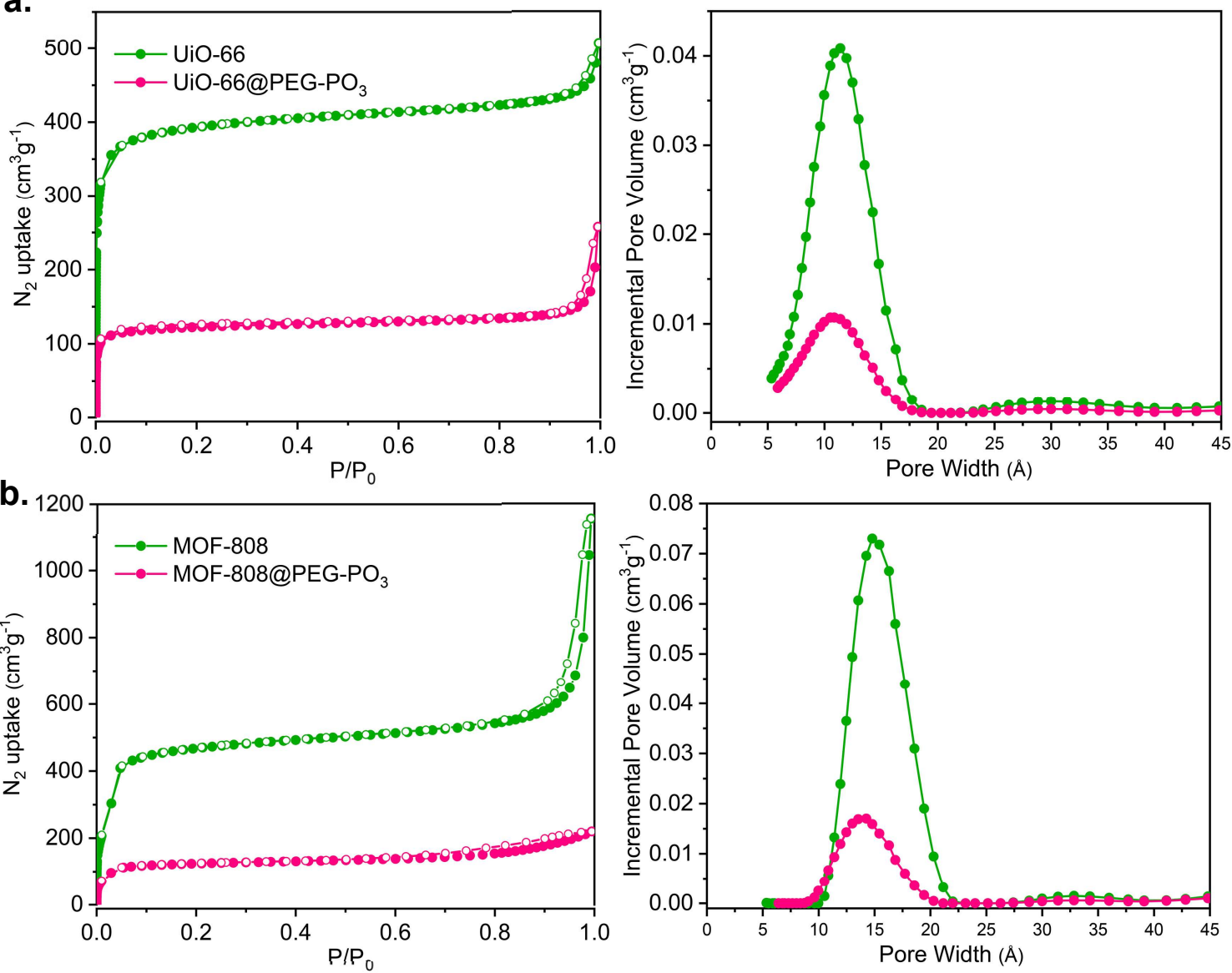

C.
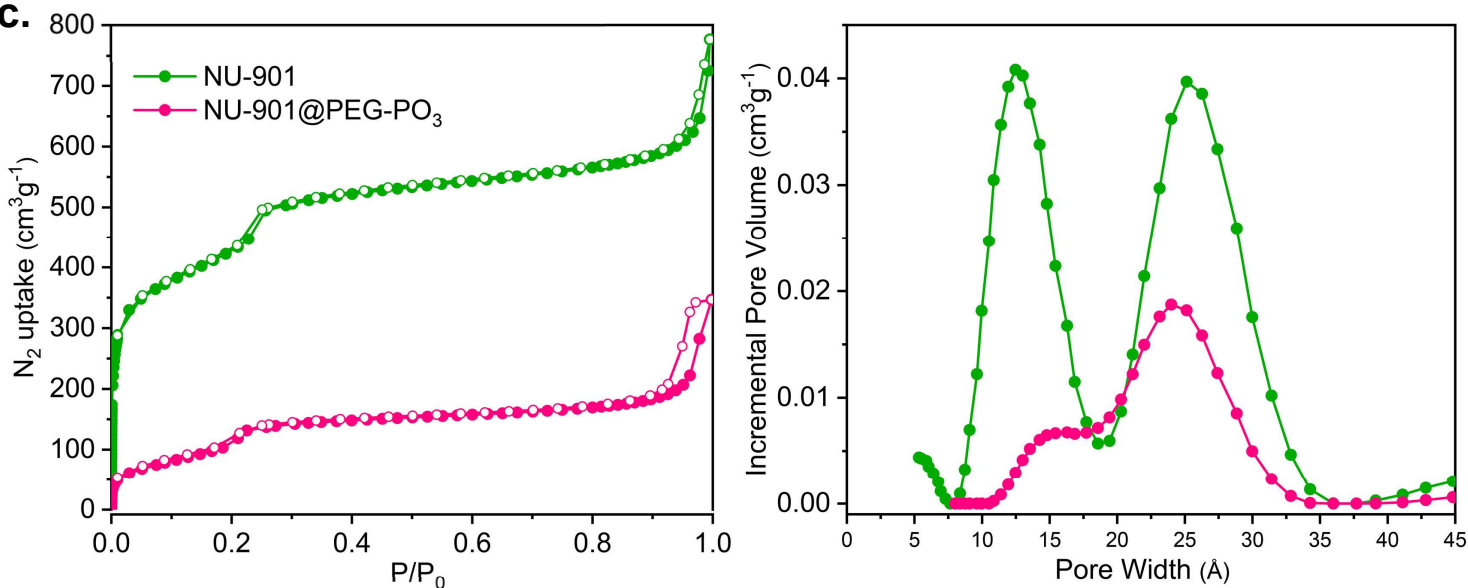

d.
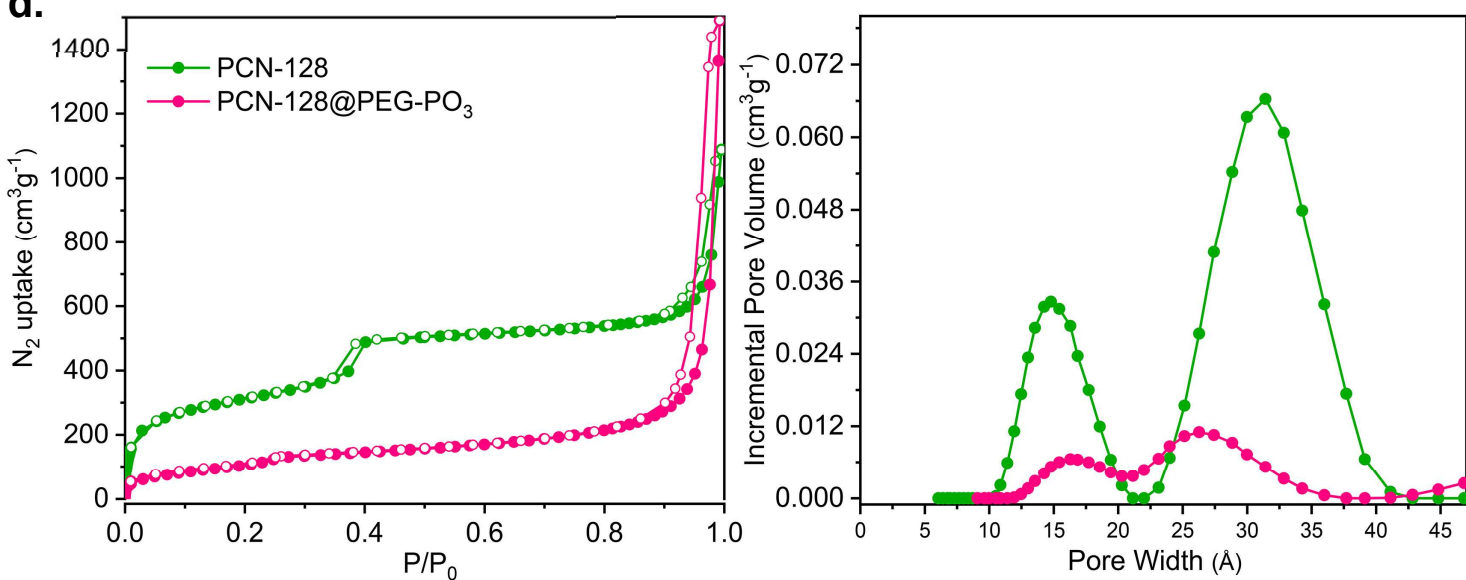

Figure S39. $\mathrm{N}_{2}$ adsorption isotherms and PSD of MOF@PEG-PO3. a. UiO-66, b. MOF-808, c. NU-901 and d. PCN-128. 
a.

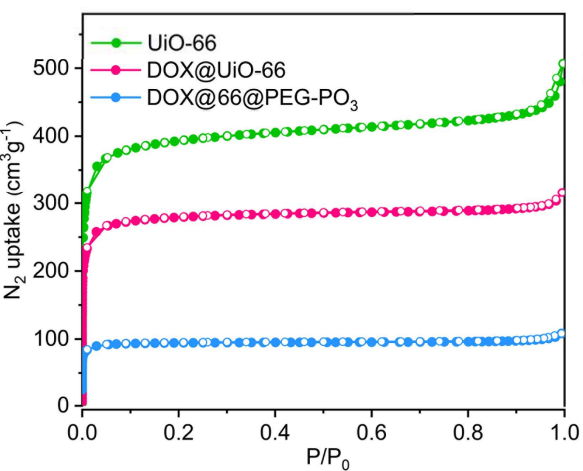

b.
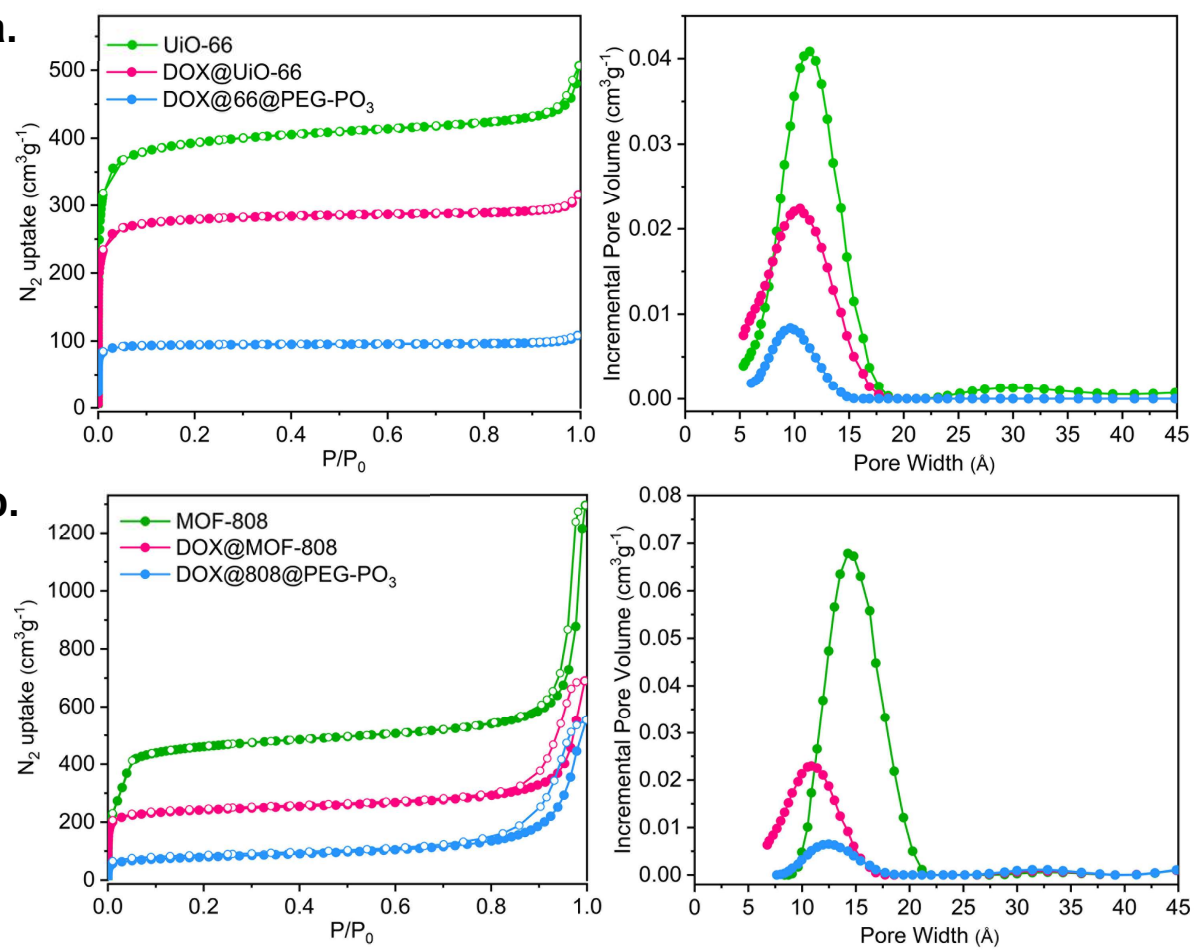

c.
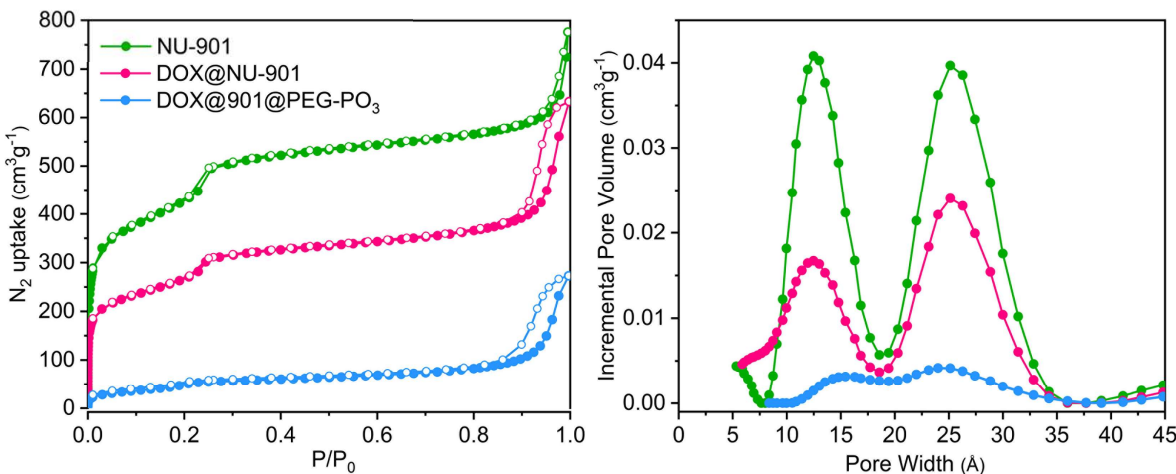

d.
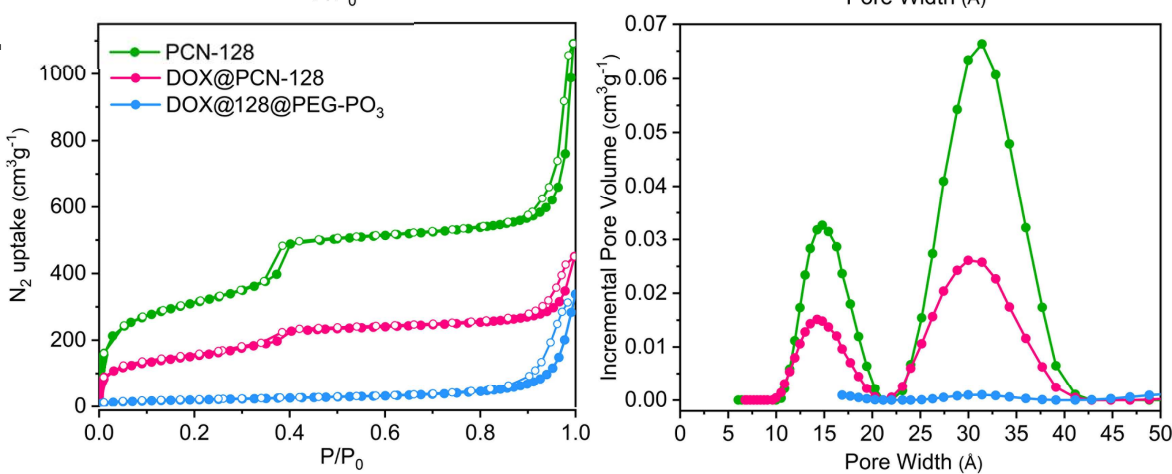

e.
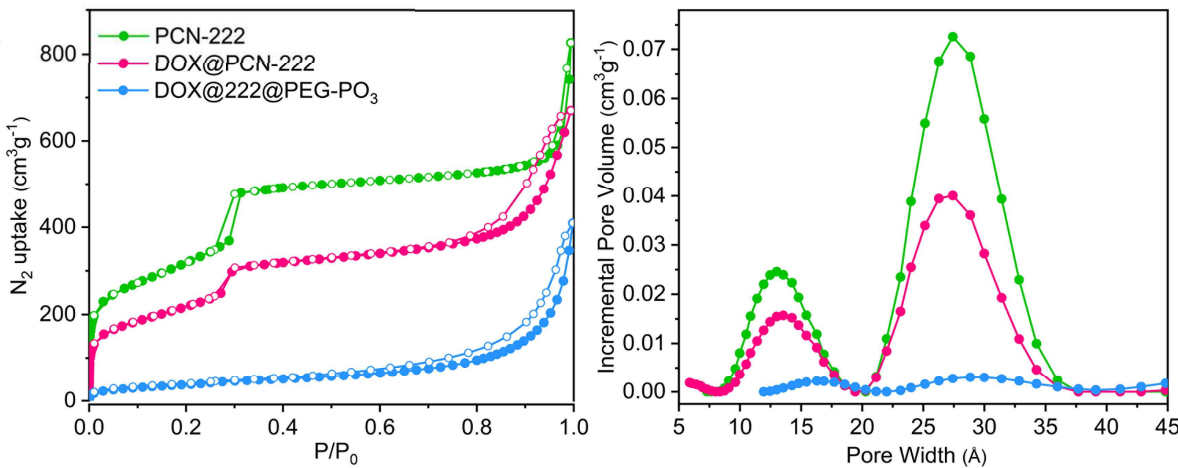

Figure S40. $\mathrm{N}_{2}$ adsorption isotherms and PSD of DOX@MOF and DOX@MOF@PEG-PO3. a. UiO-66, b. MOF-808, c. NU-901, d. PCN-128 and e. PCN-222. 


\section{DLS and zeta potential}
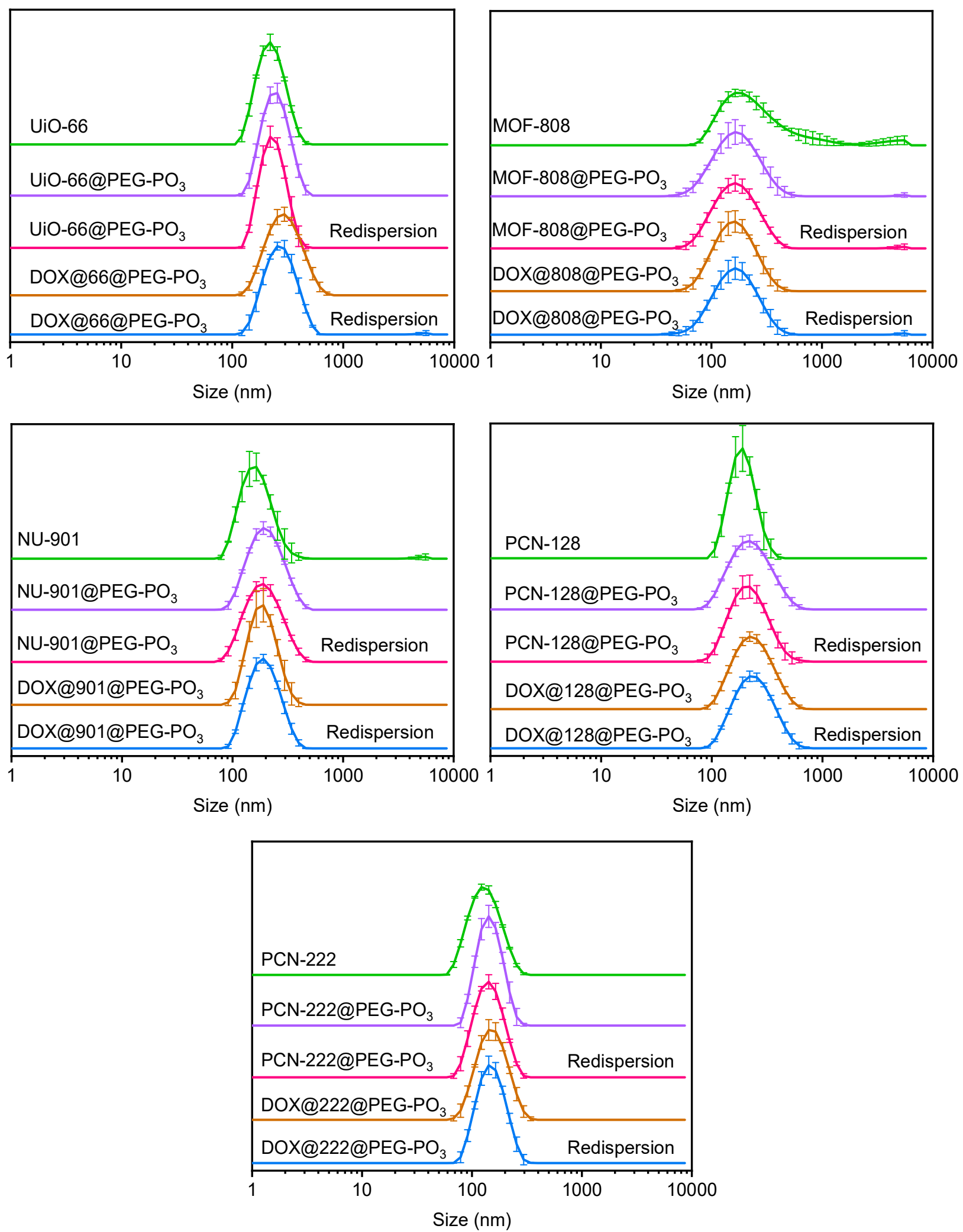

Figure S41. Intensity-average diameter of the suspension of parent nanoMOFs (green line), MOF@PEG-PO 3 before (purple line) and after (pink line) lyophilization, DOX@MOF@PEG-PO 3 before (brown line) and after (blue line) lyophilization $(n=3)$. 


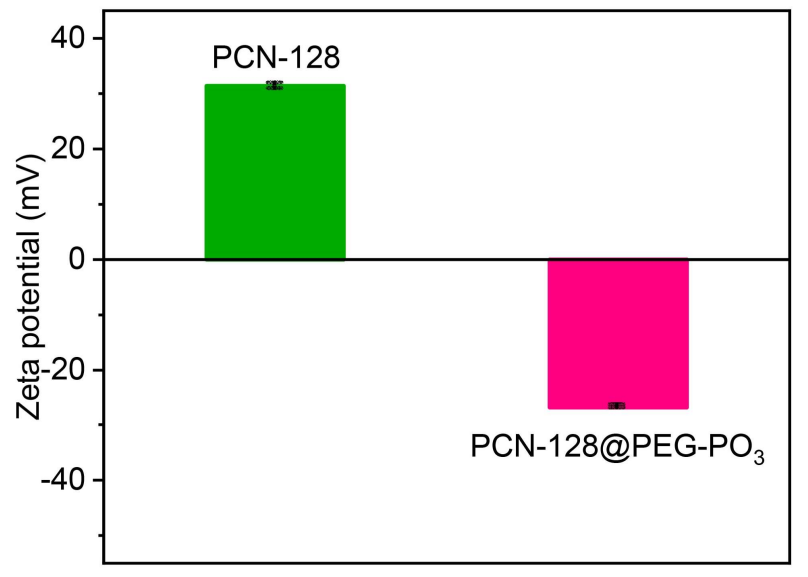

Figure S42. Zeta potential of PCN-128 and PCN-128@PEG-PO
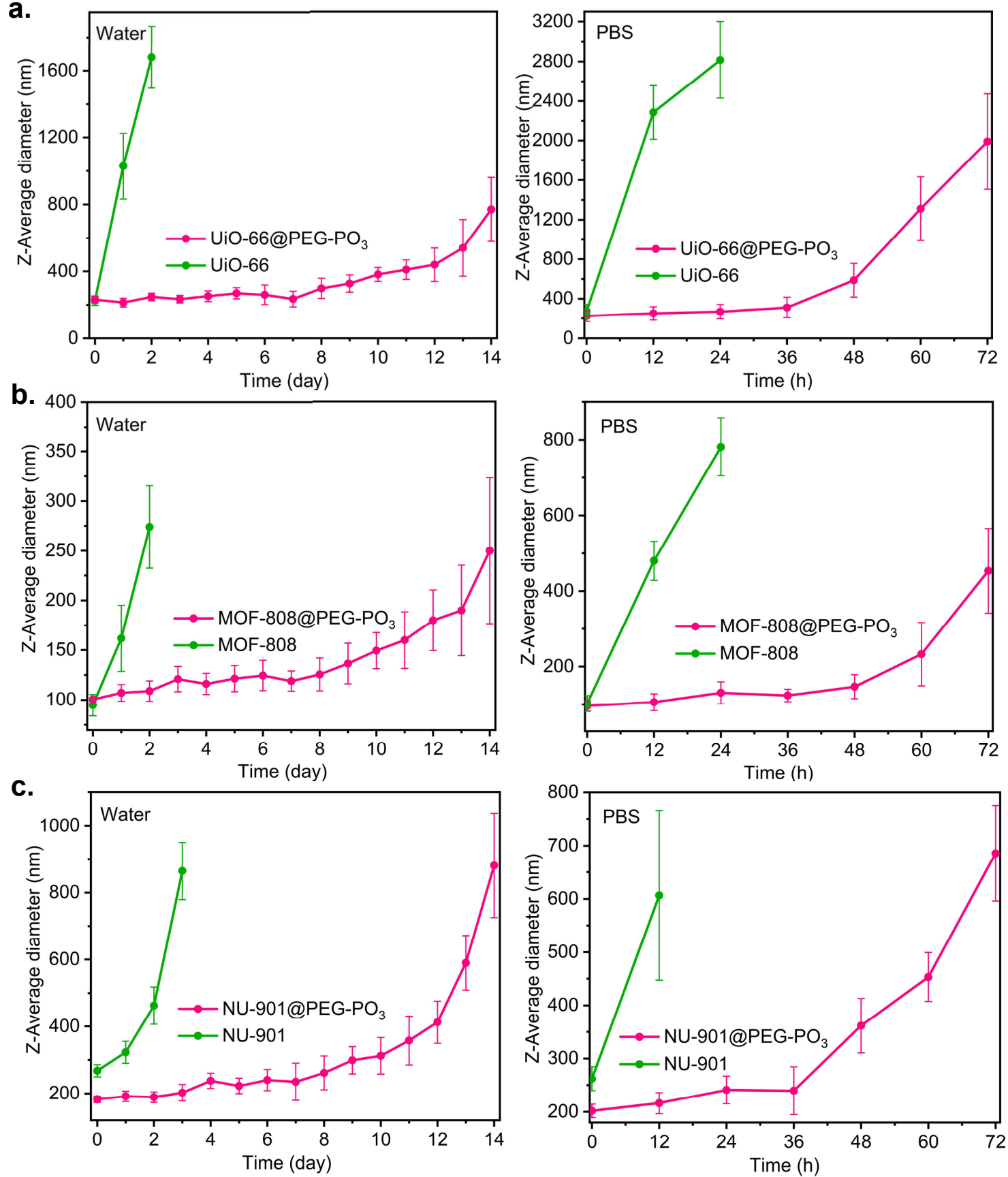

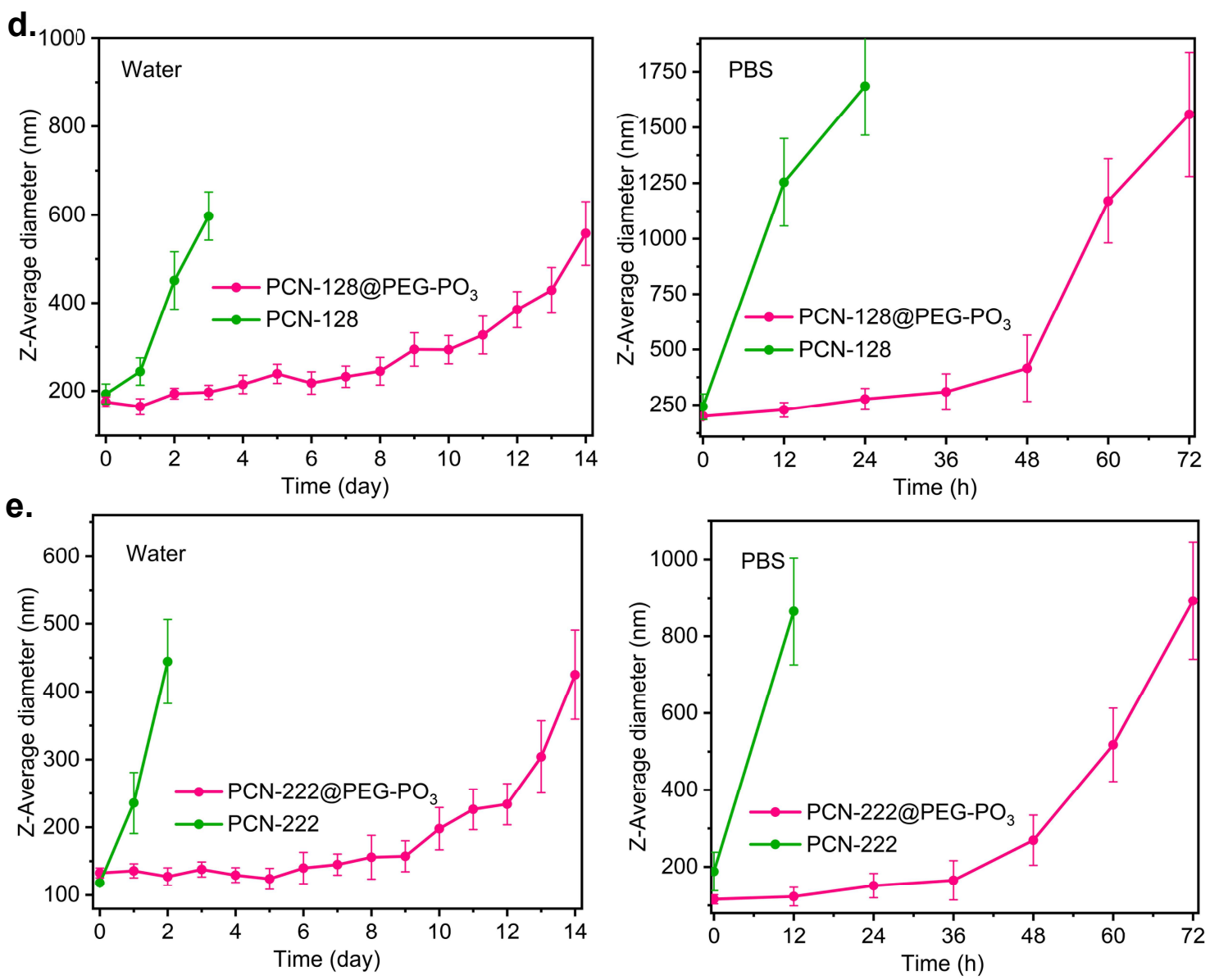

Figure S43. Long-term dispersity of bare MOFs (green line) and MOF@PEG-PO ${ }_{3}$ suspended in $\mathrm{H}_{2} \mathrm{O}$ or PBS $(\mathrm{pH}=7.4)$. a. UiO-66, b. MOF-808, c. NU-901, d. PCN-128 and e. PCN-222 The sizes were evaluated by DLS $(n=3)$. During the period of measurement, the samples were left on the tube roller. 

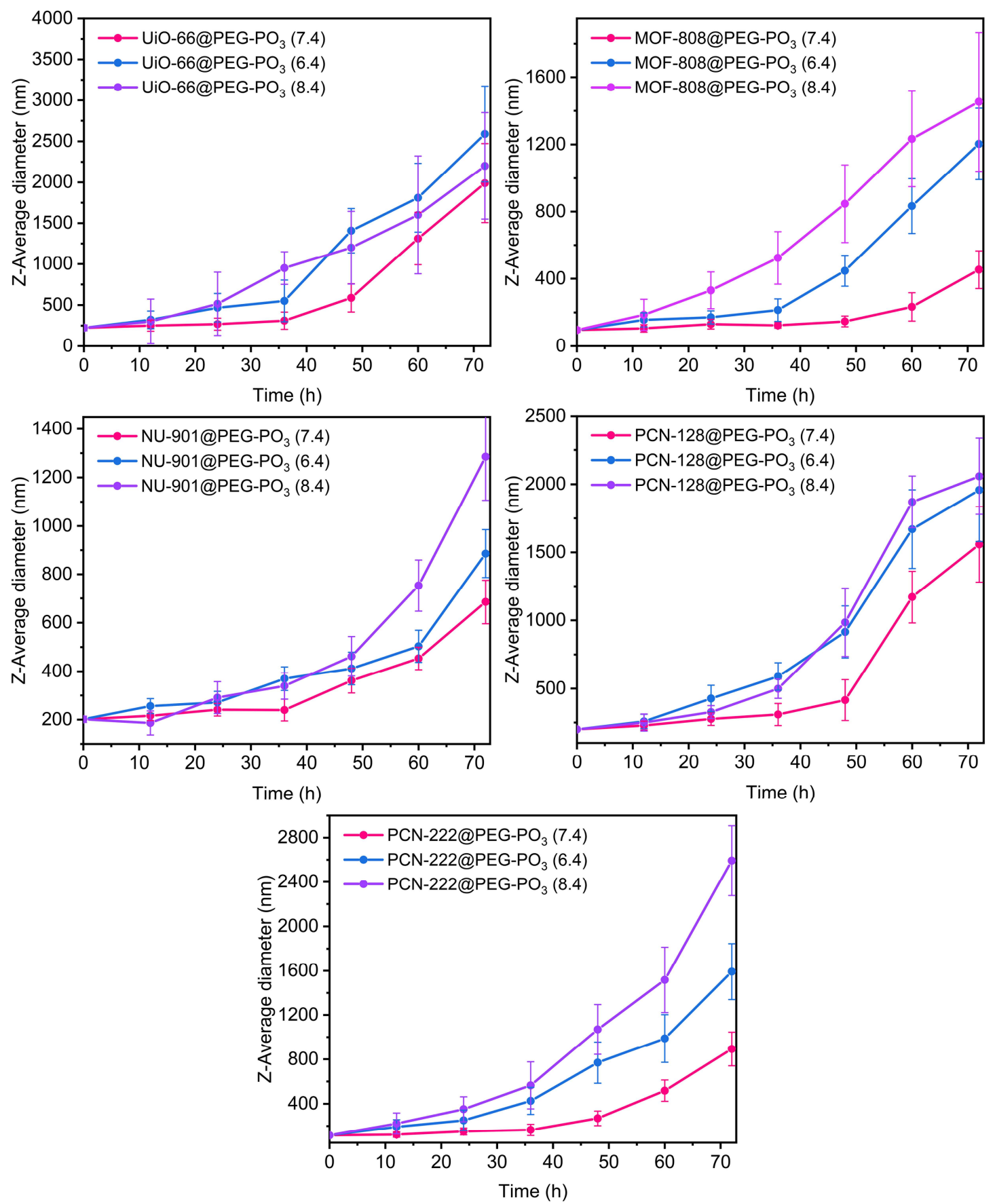

Figure S44. Long-term dispersity of MOF@PEG-PO 3 suspended in PBS with different $\mathrm{pH}$ values. Sizes were evaluated by DLS $(n=3)$. During the period of measurement, the samples were left on the tube roller. 
DOX release studies in PBS $(\mathrm{pH}=7.4)$ and water
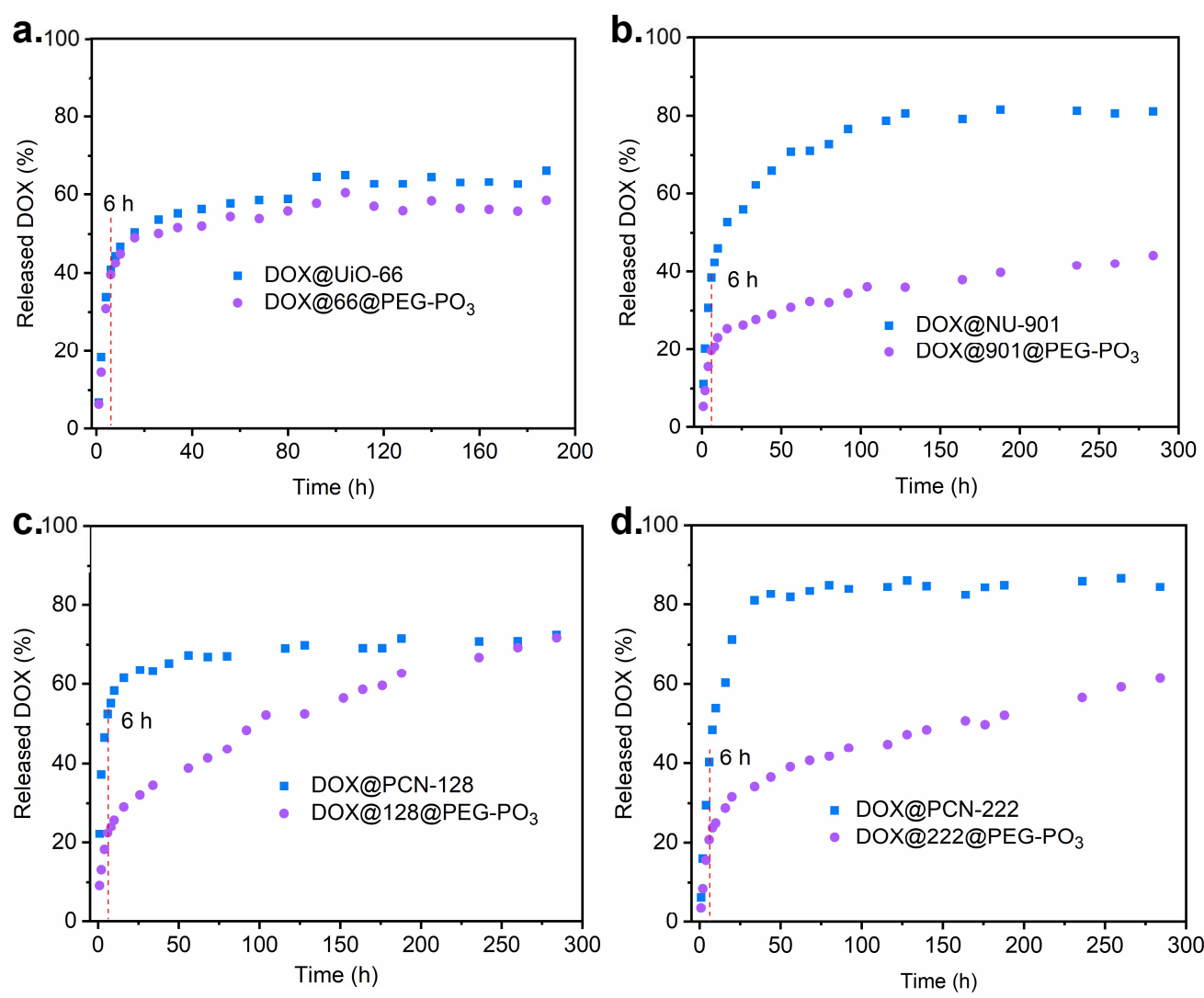

Figure S45. DOX-release profiles from a. bare and PEGylated UiO-66, b. bare and PEGylated NU-901, c. bare and PEGylated PCN-128, d. bare and PEGylated PCN-222, in PBS (pH 7.4). 


\section{S4. BET area calculation using BETS| ${ }^{23}$}

BETSI Analysis for PCN-222
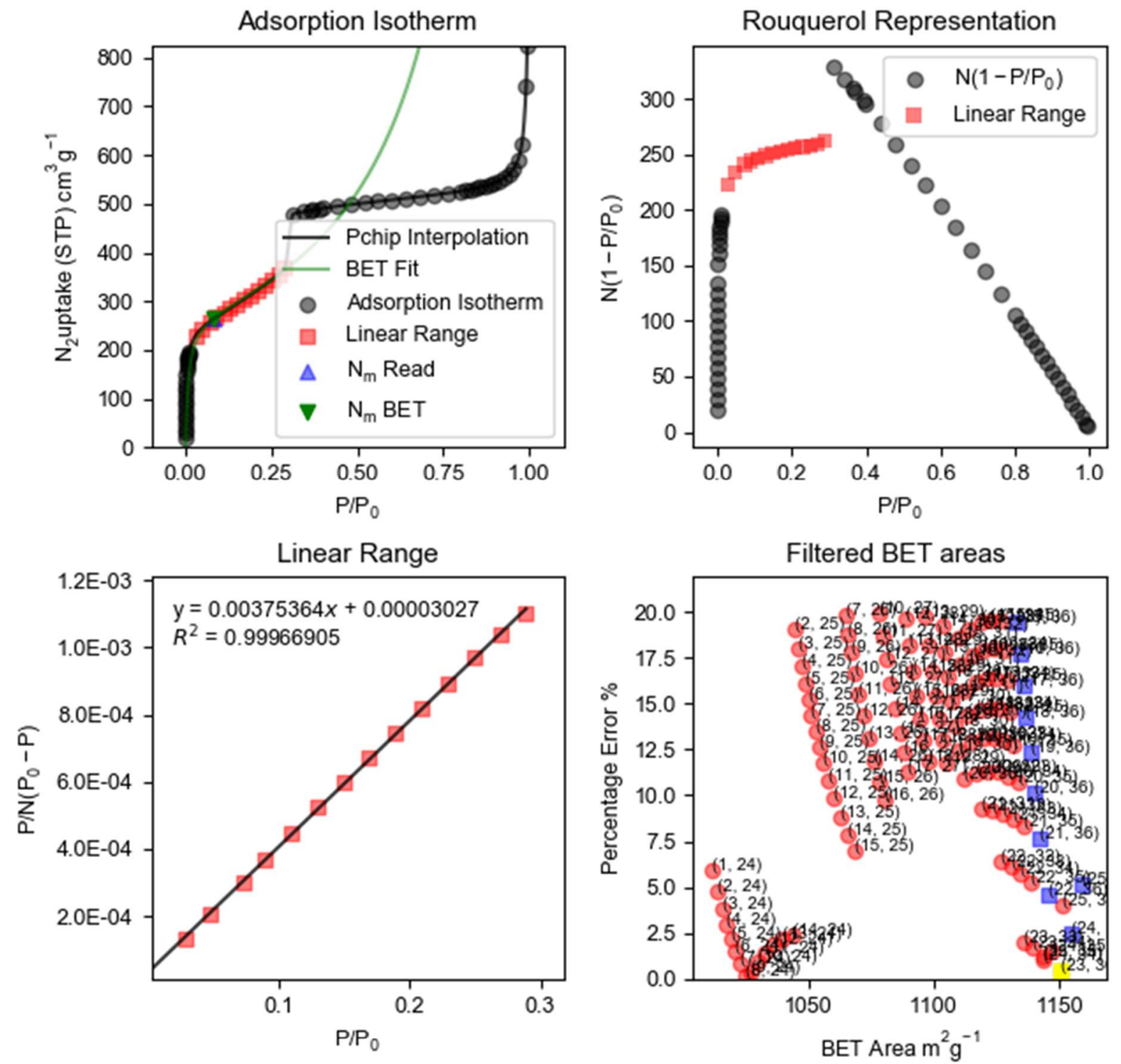

Filtered Monolayer-Loadings
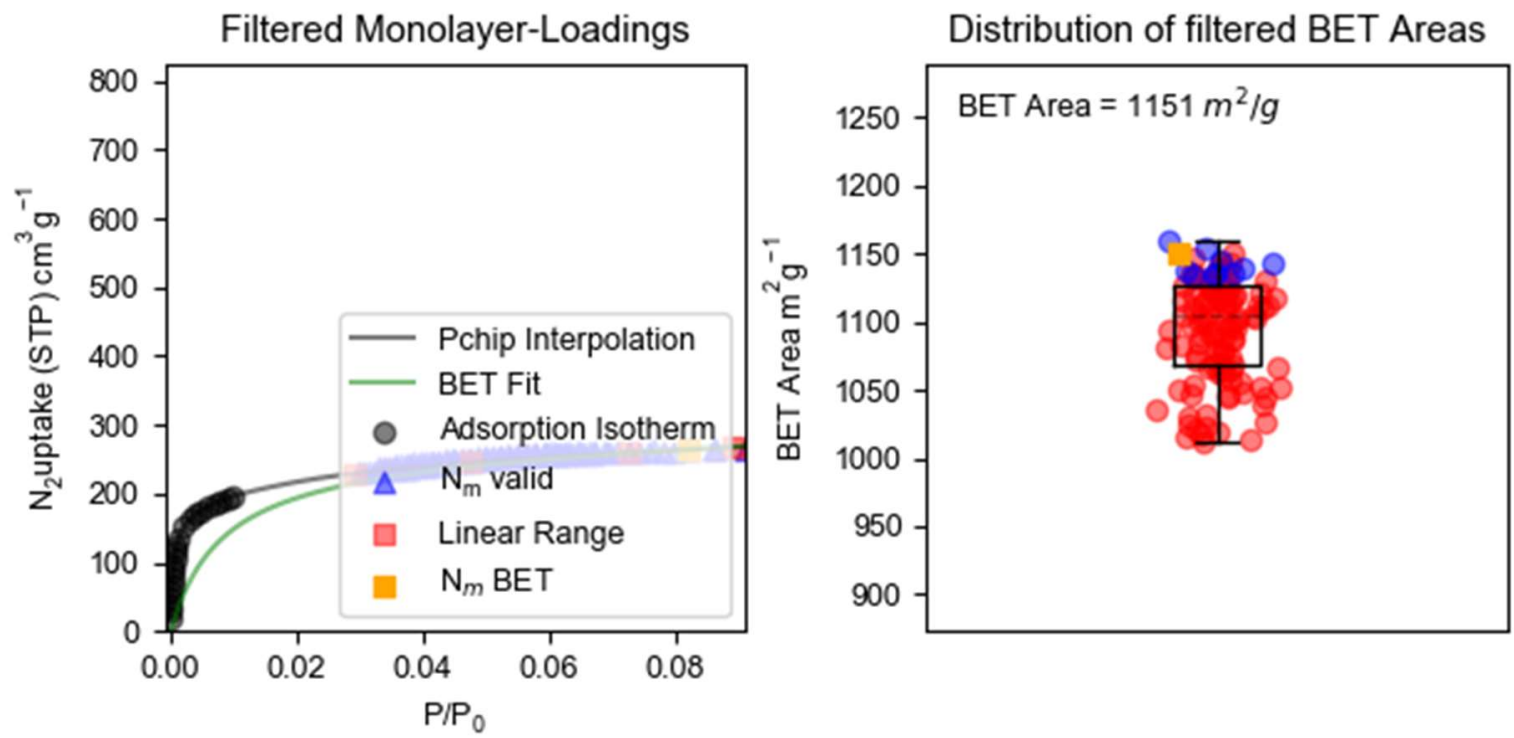
BETSI Regression Diagnostics for PCN-222
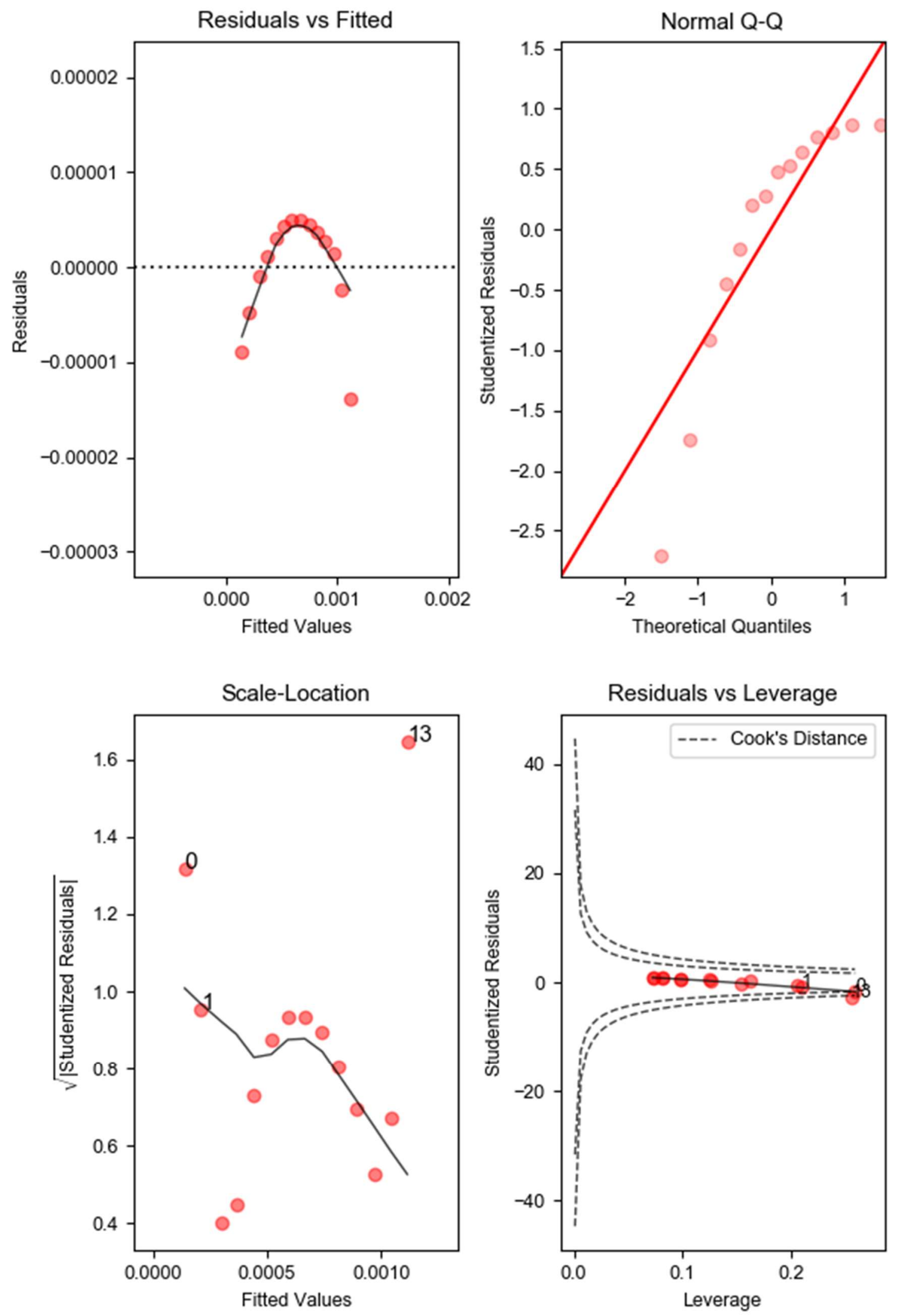
BETSI Analysis for PEGylated-2h
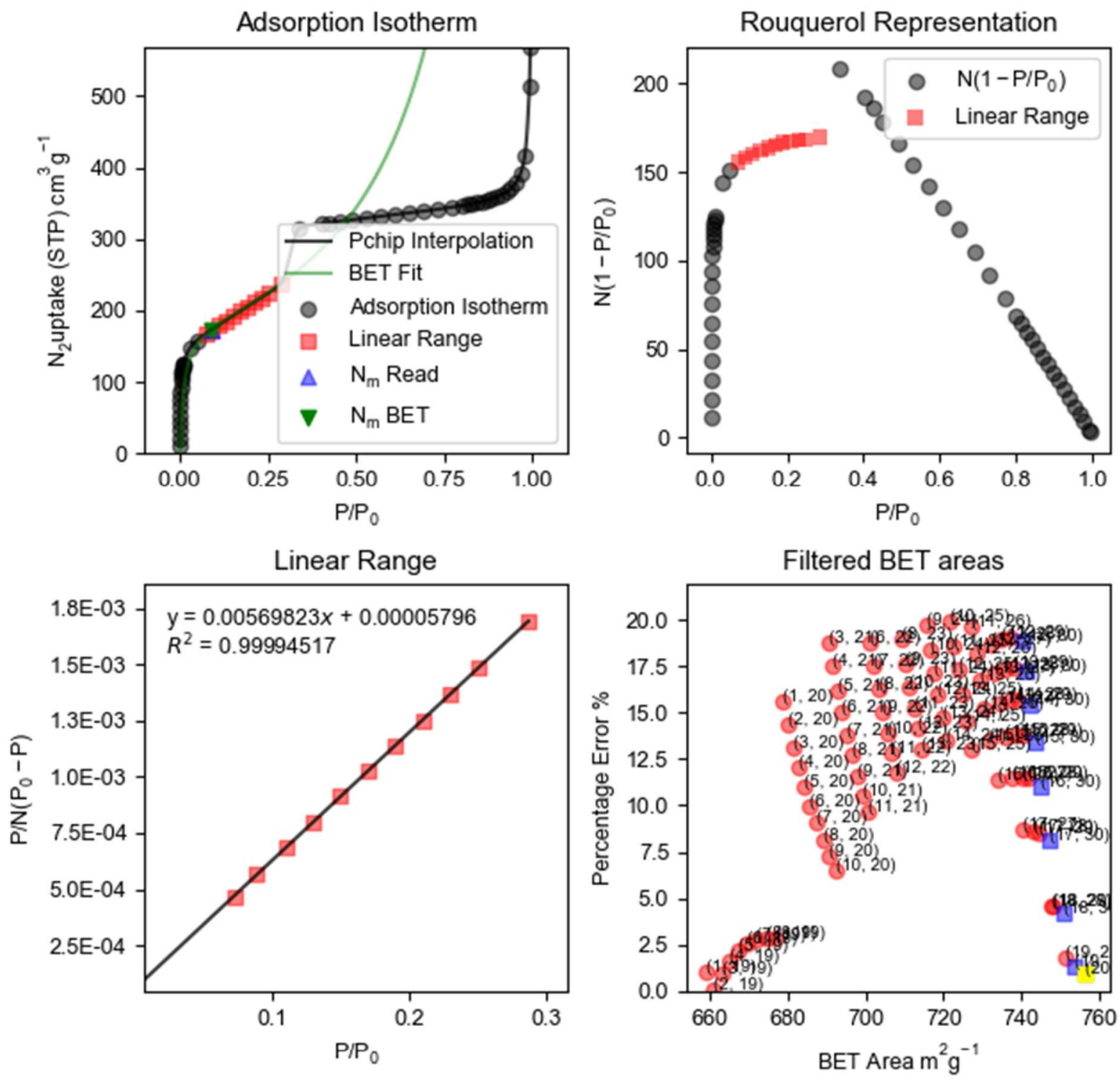

Filtered Monolayer-Loadings
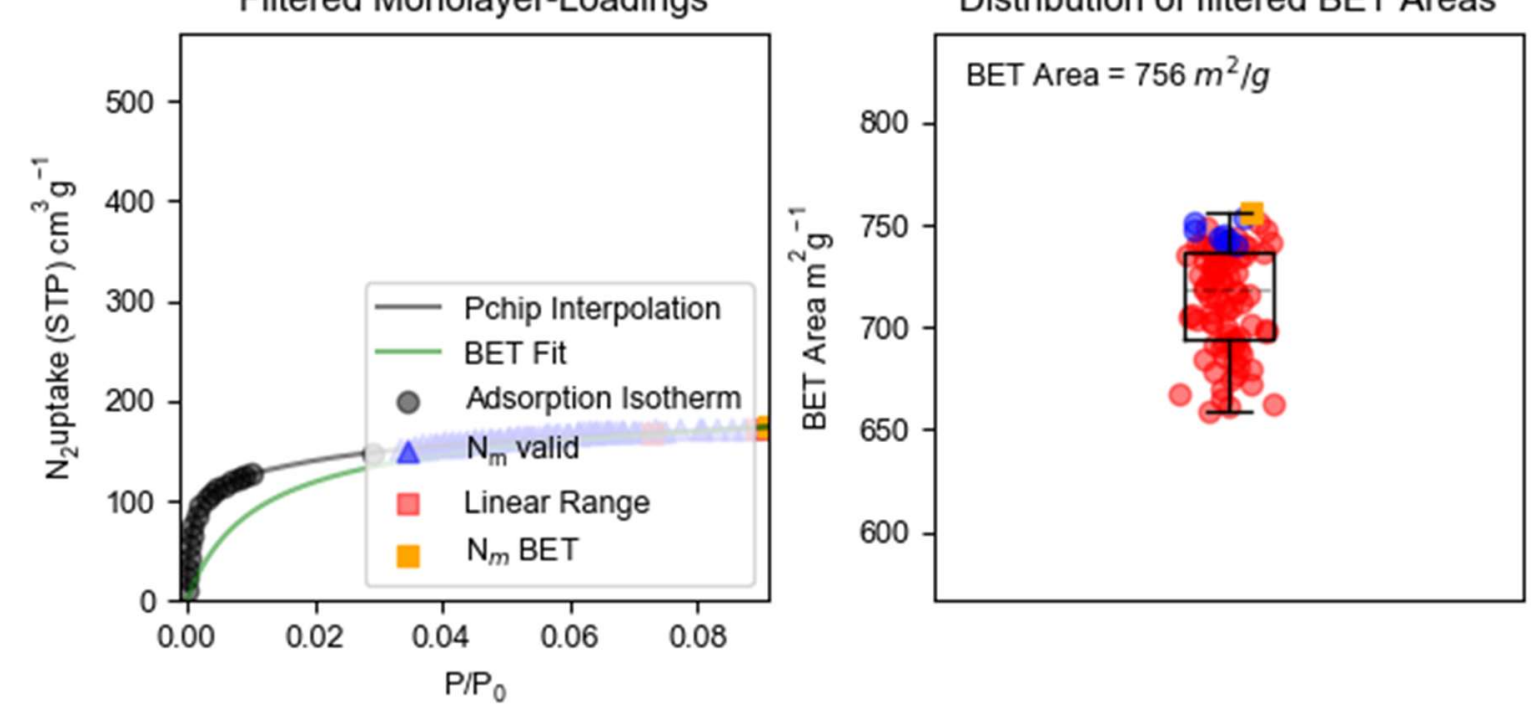
BETSI Regression Diagnostics for PEGylated-2h
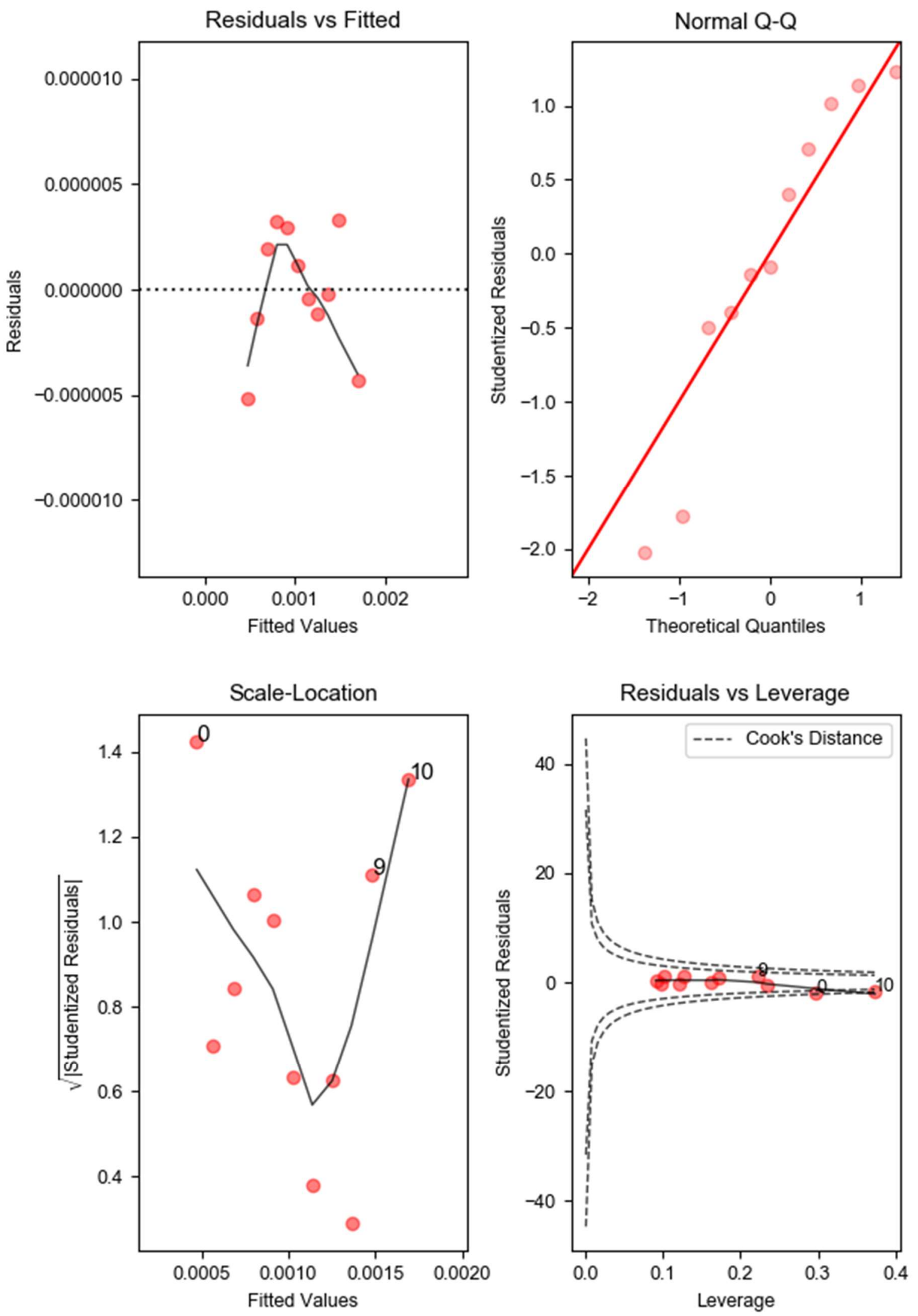
BETSI Analysis for PEGylated-4h
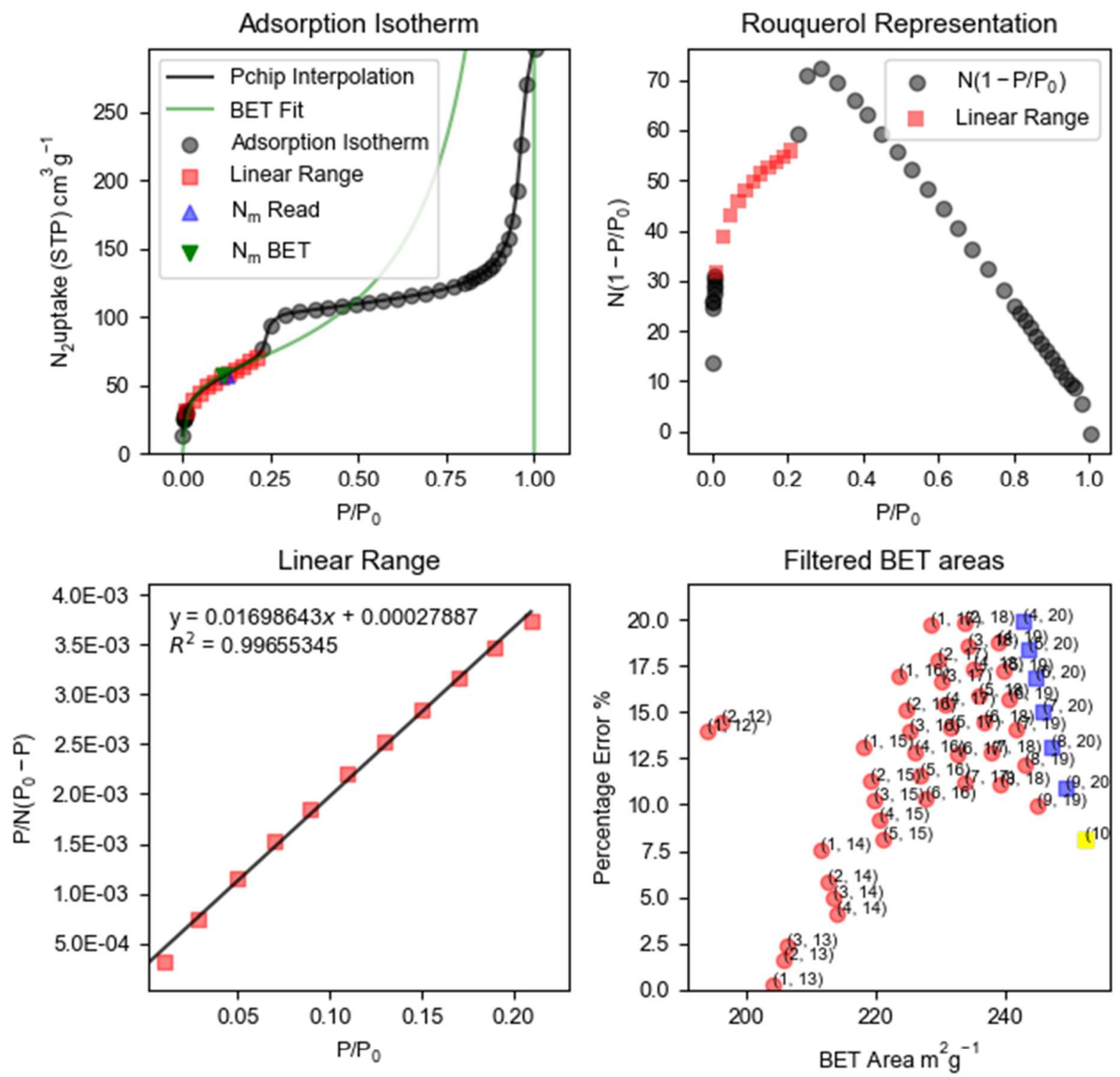

Filtered Monolayer-Loadings
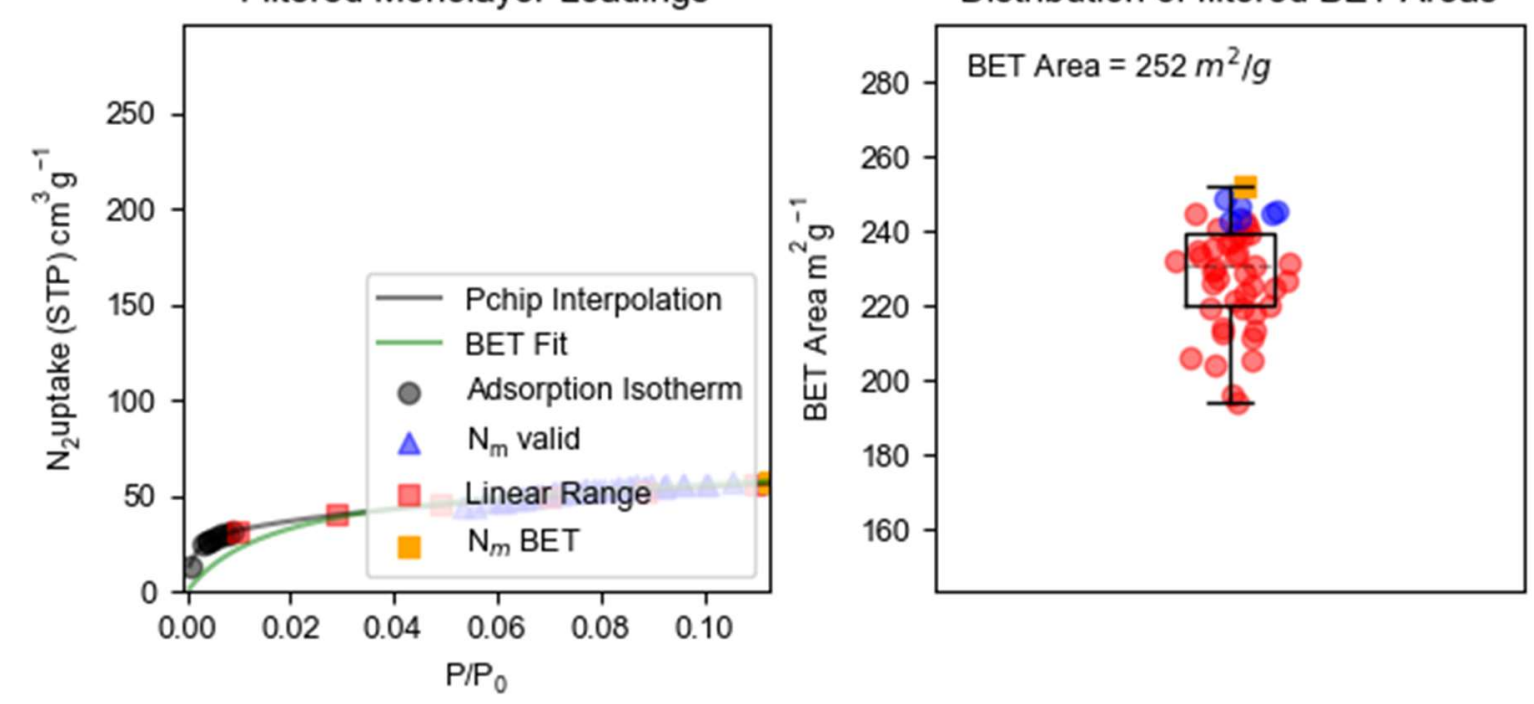
BETSI Regression Diagnostics for PEGylated-4h
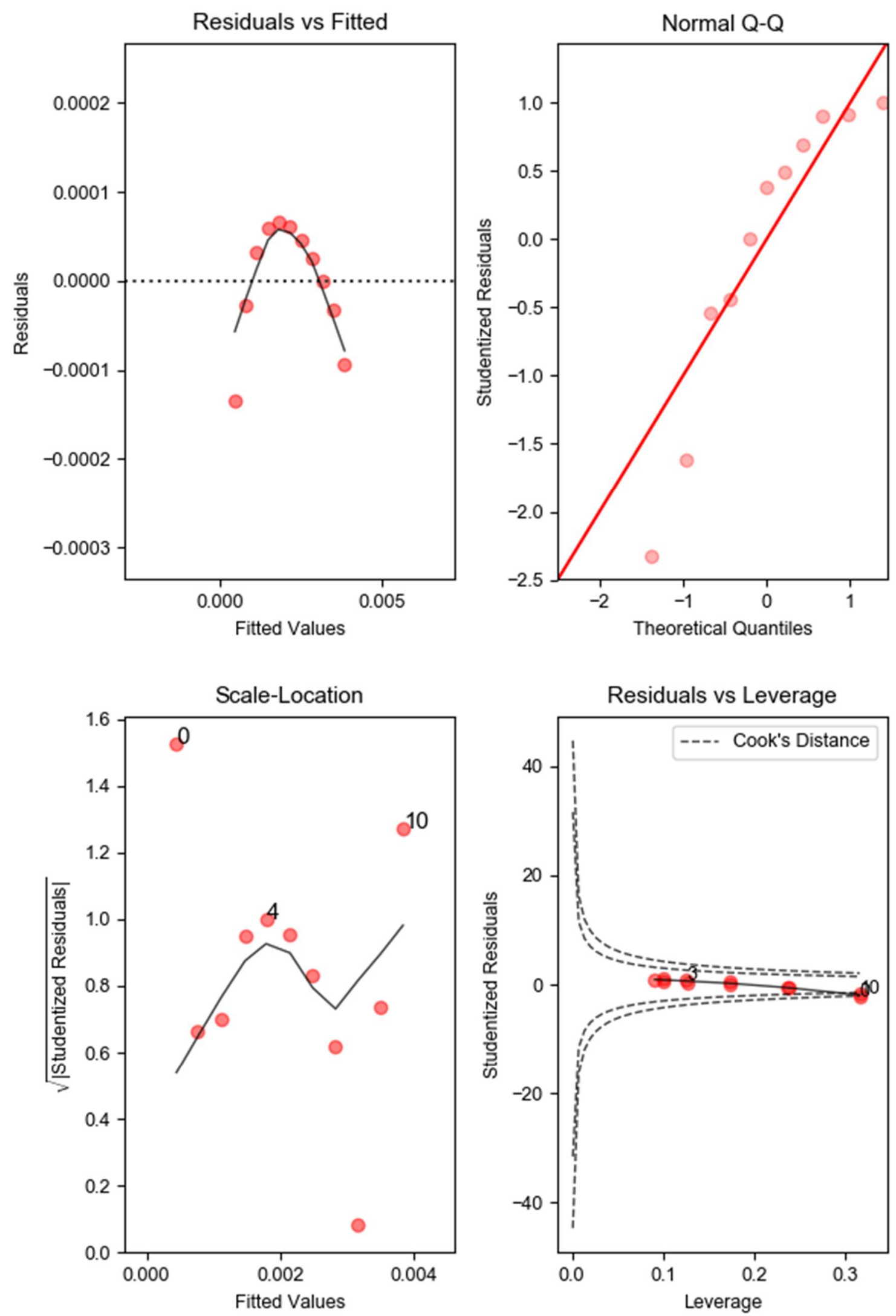


\section{BETSI Analysis for PEGylated-12h}
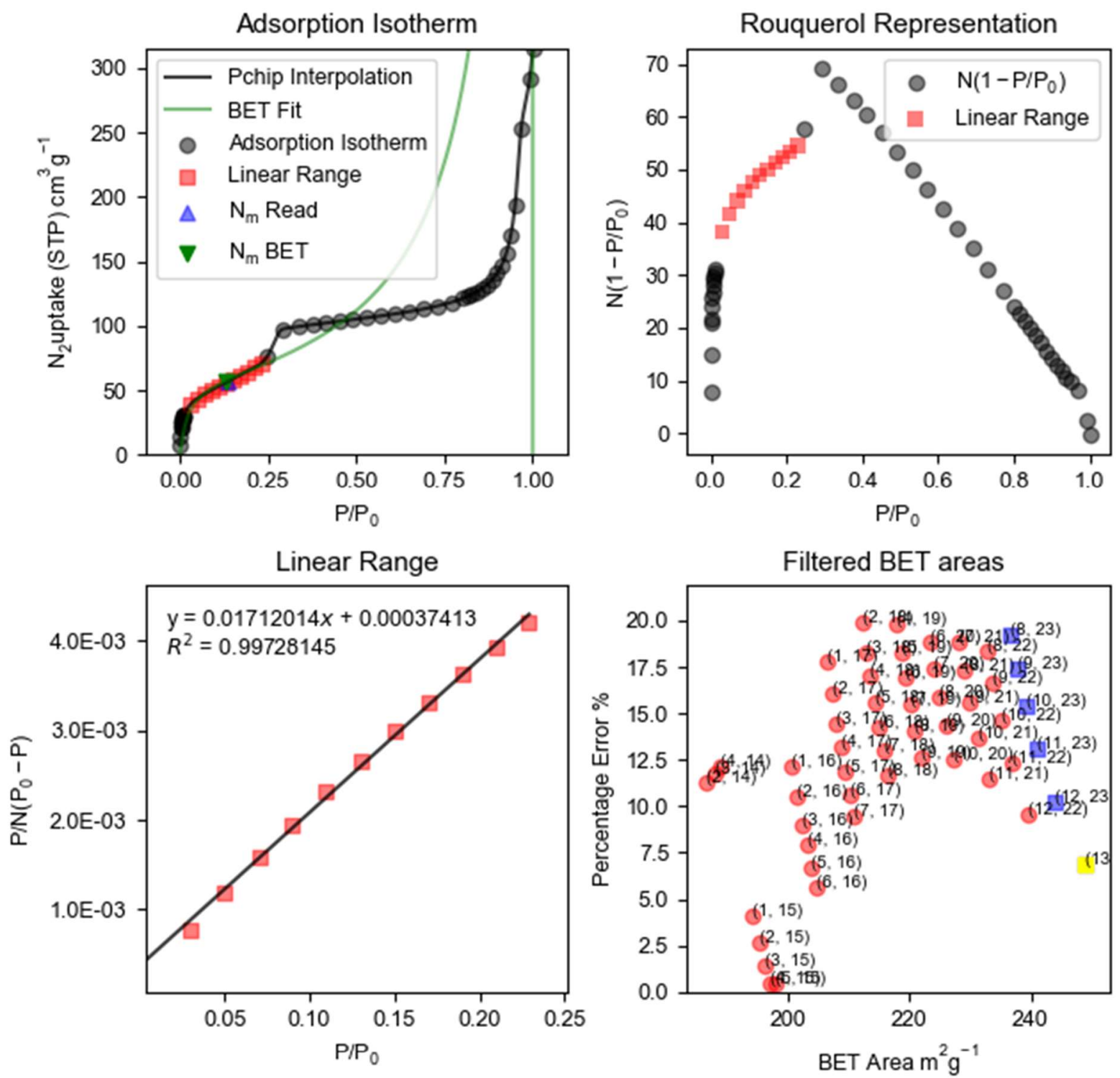

Filtered Monolayer-Loadings

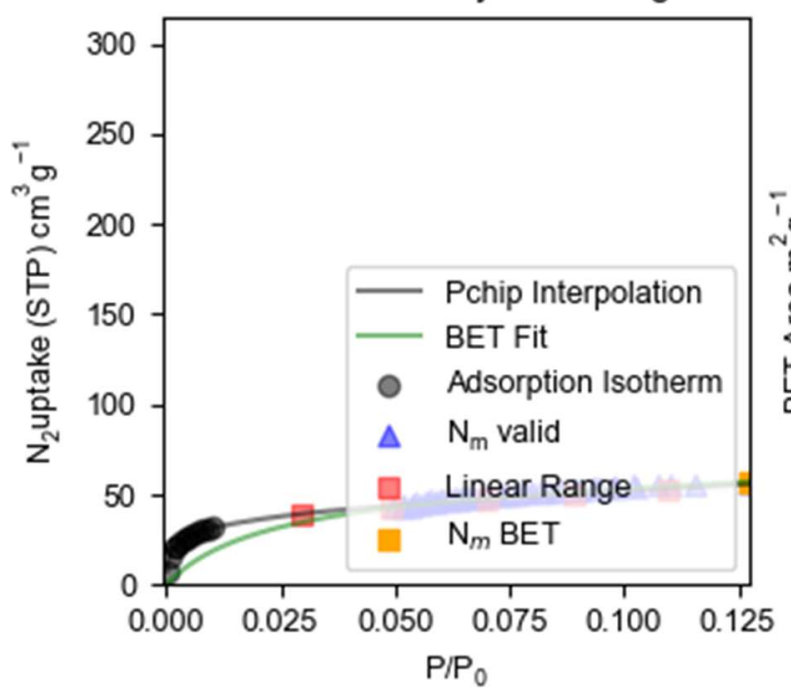

Distribution of filtered BET Areas

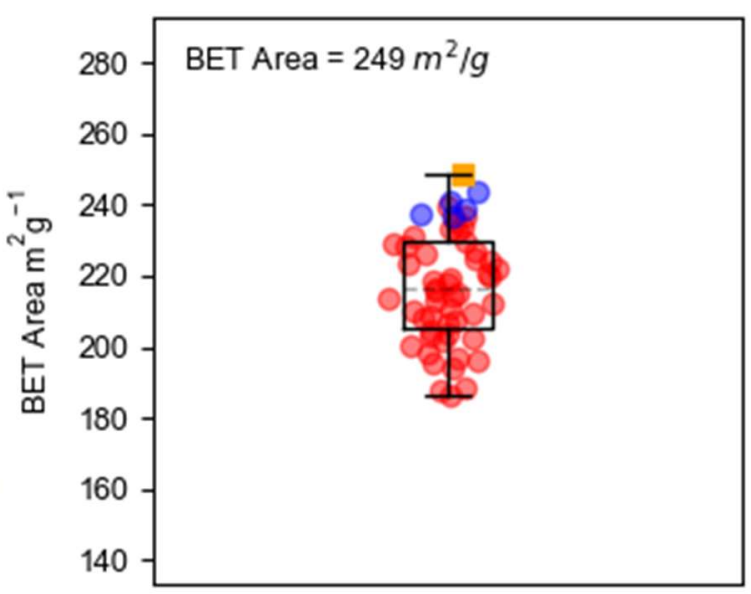


BETSI Regression Diagnostics for PEGylated-12h
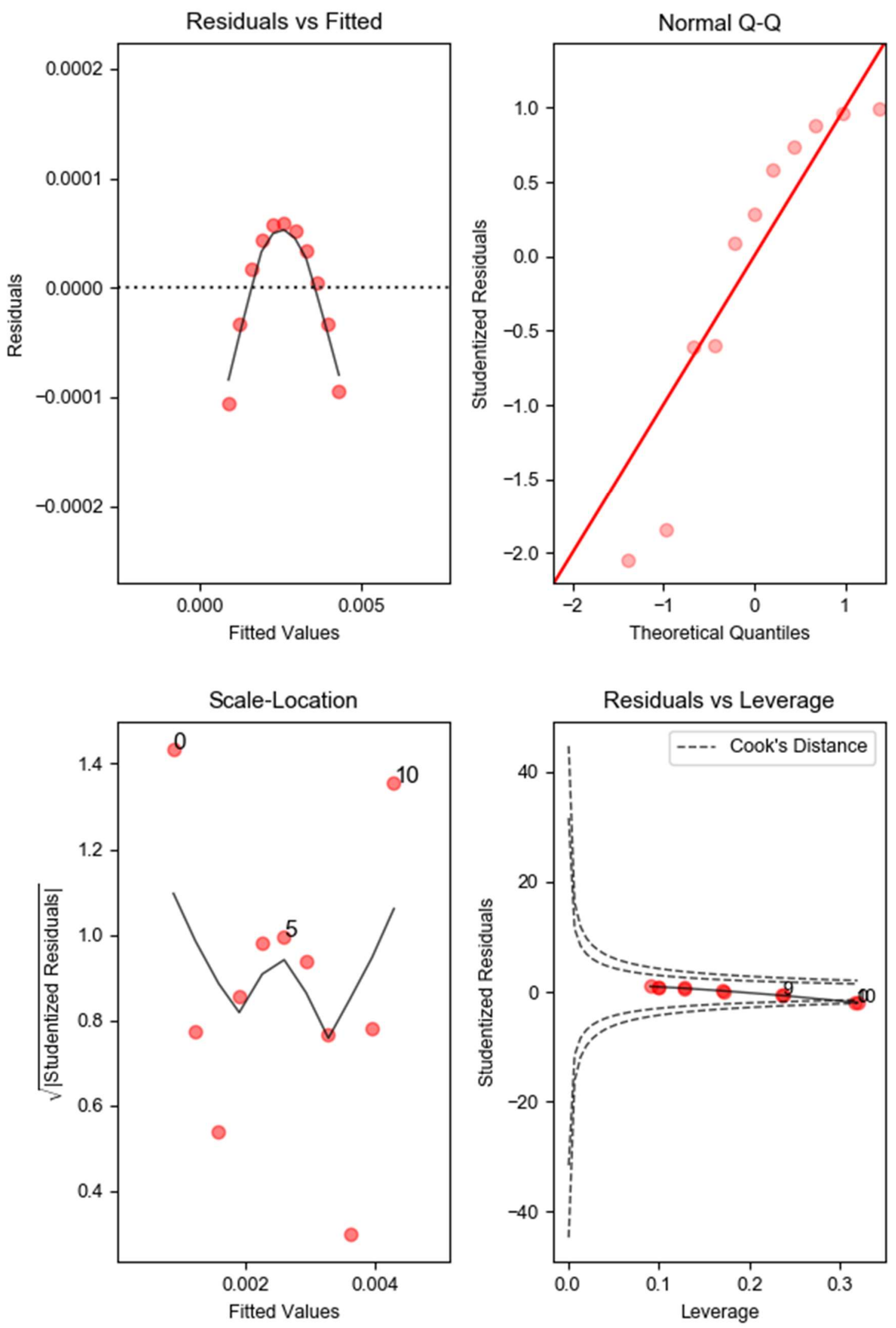
BETSI Analysis for PEGylated-16h
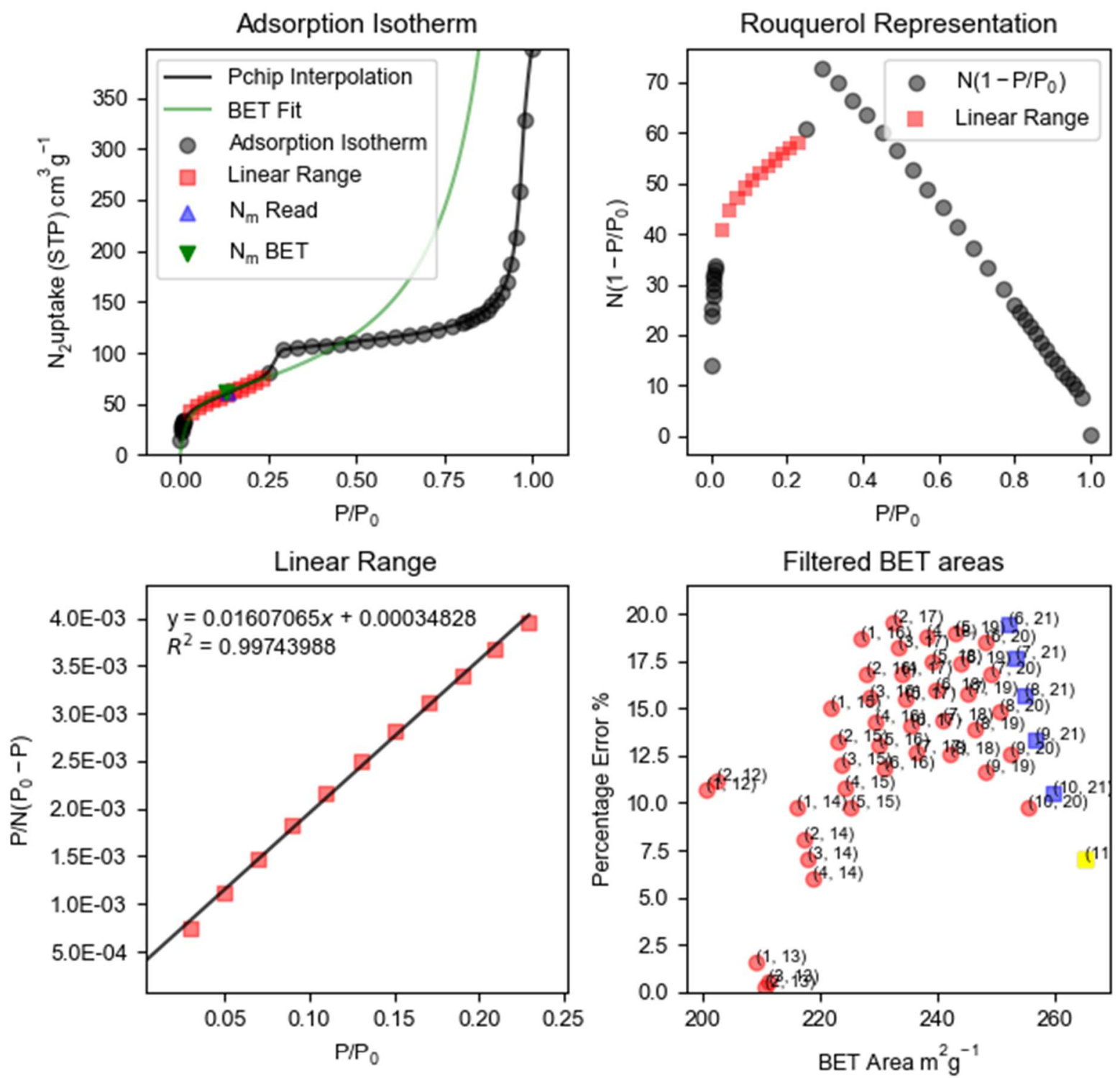

Filtered Monolayer-Loadings

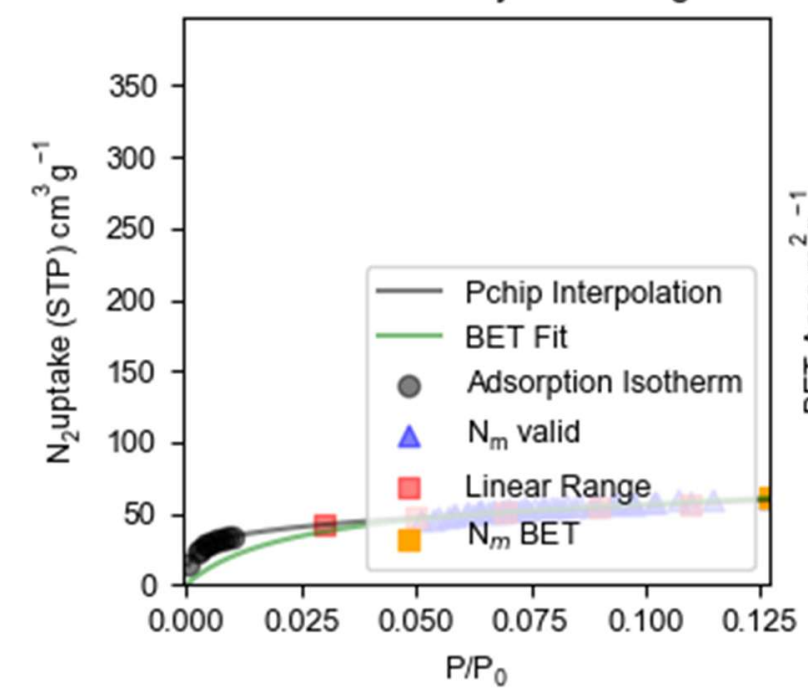

Distribution of filtered BET Areas

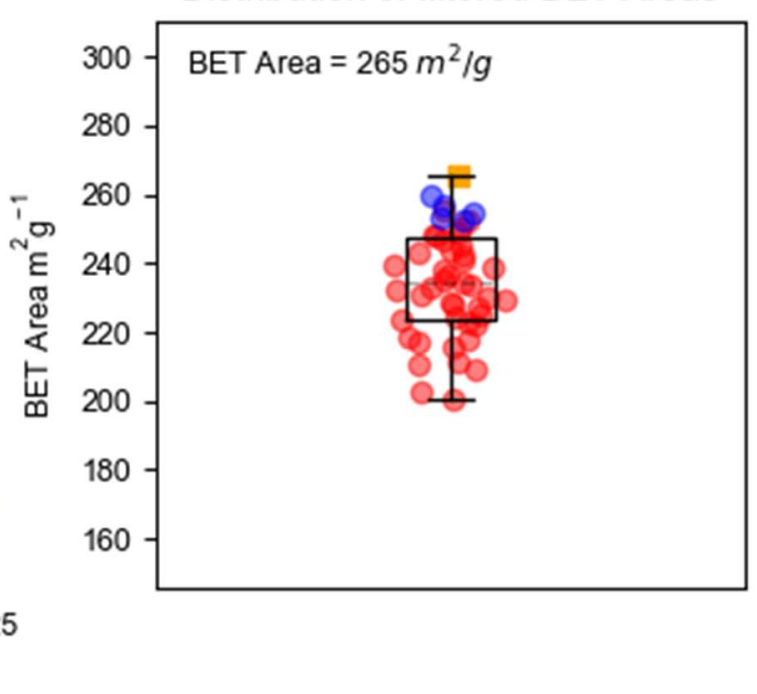


BETSI Regression Diagnostics for PEGylated-16h
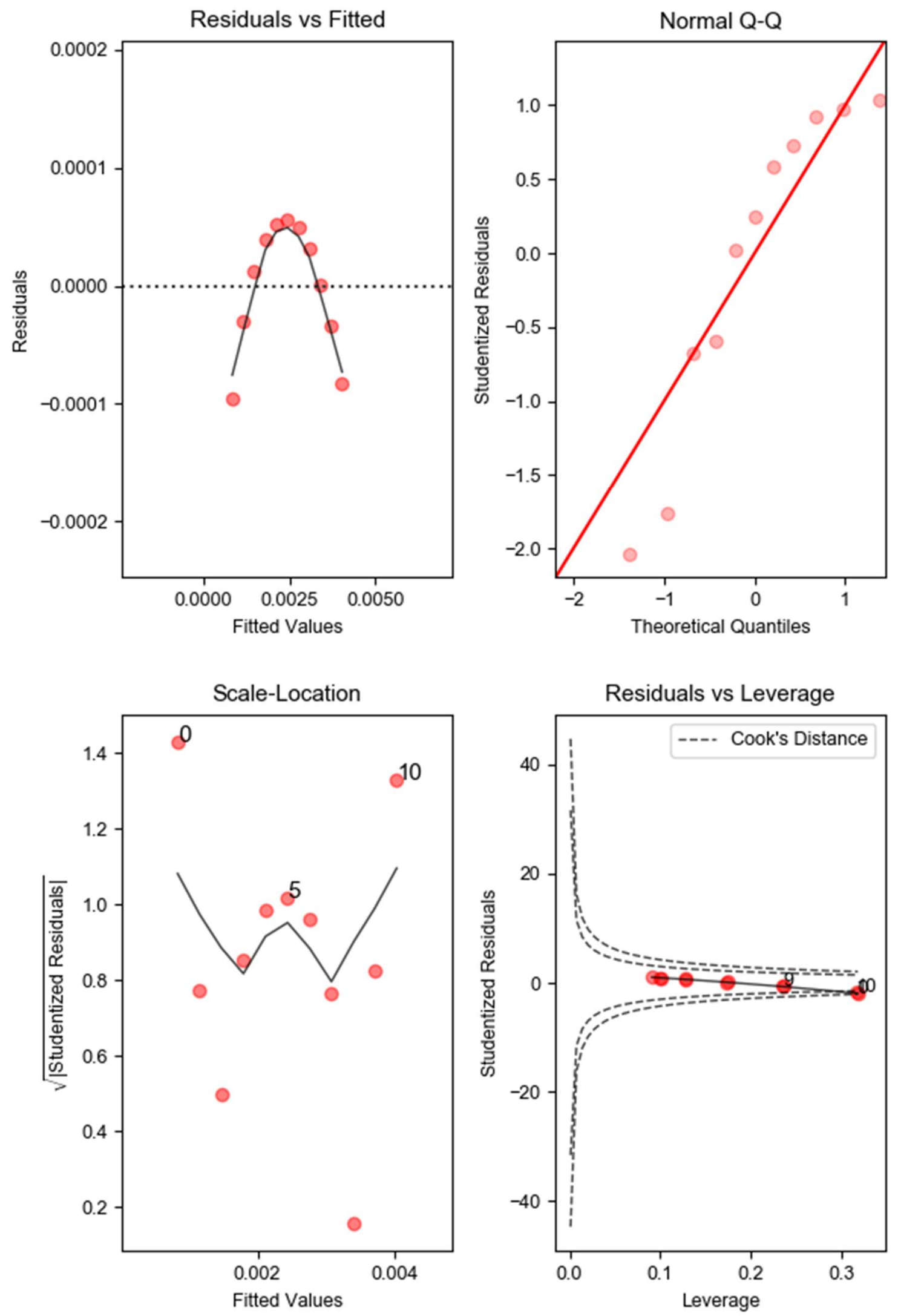


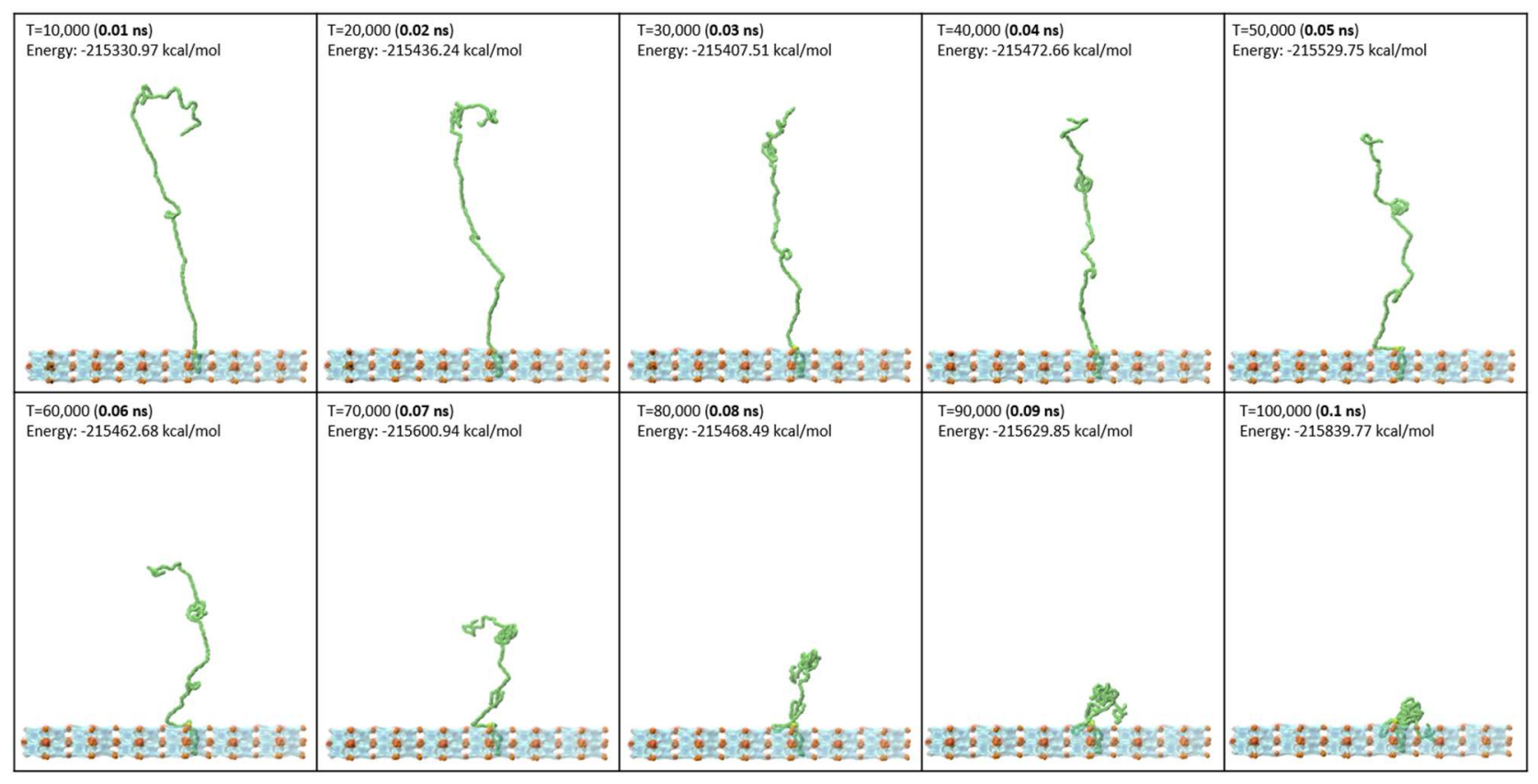

Figure S46. Position of the mPEG-PO 3 relative to the framework as a function of time. The total energy of the system (in $\mathrm{kcal} / \mathrm{mol}$ ) is also given at each time step. PEG atoms (minus the phosphate group) is in green; the phosphate group of the $\mathrm{mPEG}-\mathrm{PO}_{3}$ is in yellow; the framework atoms are in turquoise (translucent), and the $\mathrm{Zr}$ atoms of the framework are in brown.

a.

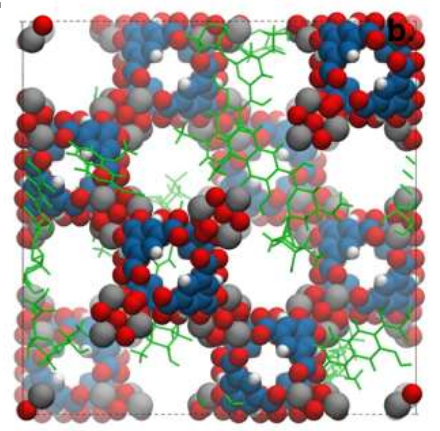

19.5 wt \%

c.

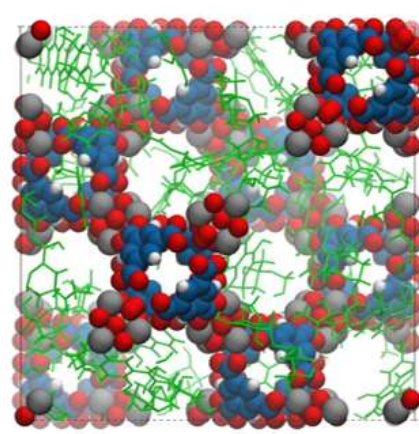

$43 \mathrm{wt} \%$ b.

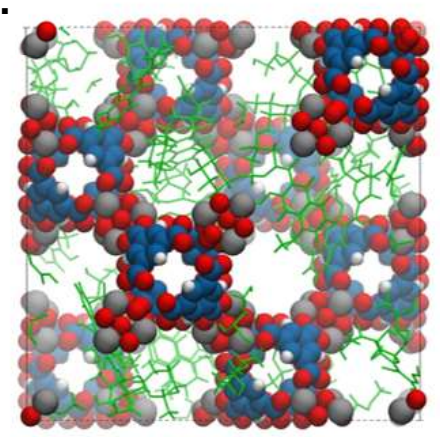

$32.6 \mathrm{wt} \%$

d.

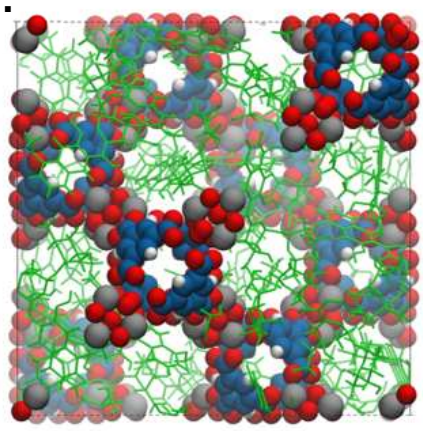

48.7 wt \%

Figure S47. Snapshots of GCMC simulations showing DOX (in green) loading inside MOF-808. a.19.5 wt\%, b. $32.6 \mathrm{wt} \%$, c. $43 \mathrm{wt} \%$, and d. $48.7 \mathrm{wt} \%$. For the framework, $\mathrm{C}$ atoms are in blue, $\mathrm{H}$ atoms in white, $\mathrm{O}$ atoms in red, and $\mathrm{Zr}$ atoms in grey. 
a.

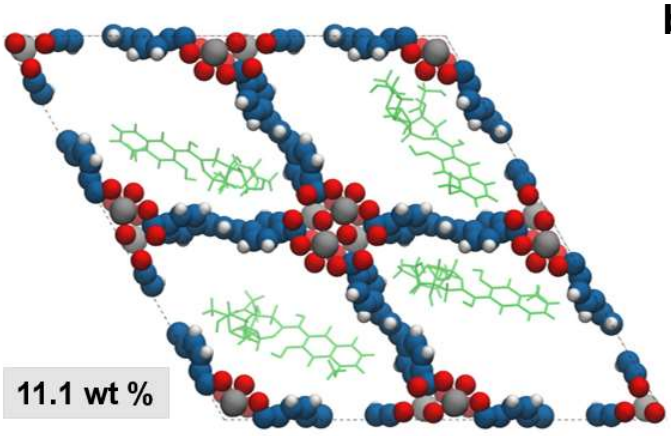

c.

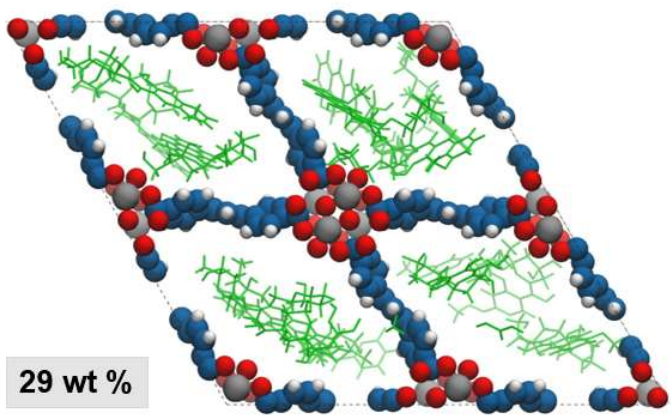

b.

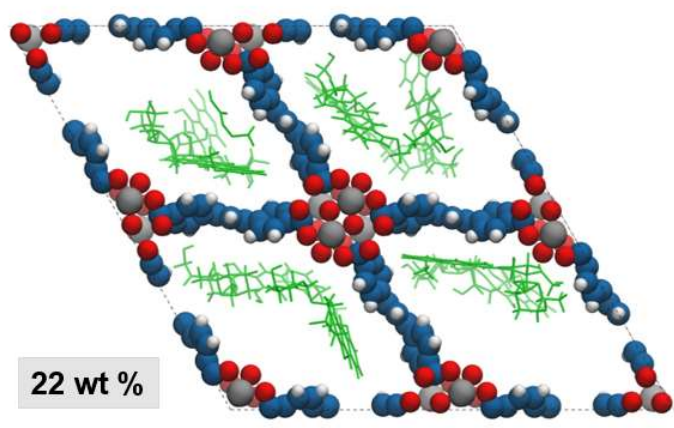

d.

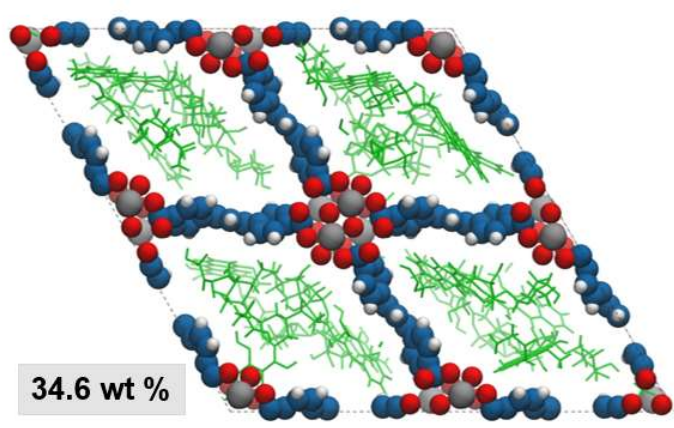

Figure S48. Snapshots of GCMC simulations showing DOX (in green) loading inside NU-901. a. 11.1 wt\%, b. $22 \mathrm{wt} \%$, c. $29 \mathrm{wt} \%$, and d. $34.6 \mathrm{wt} \%$. For the framework, $\mathrm{C}$ atoms are in blue, $\mathrm{H}$ atoms in white, $\mathrm{O}$ atoms in red, and $\mathrm{Zr}$ atoms in grey.

a.

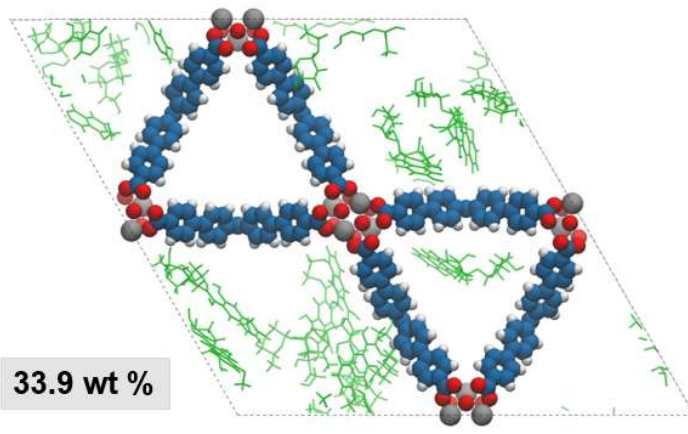

c.

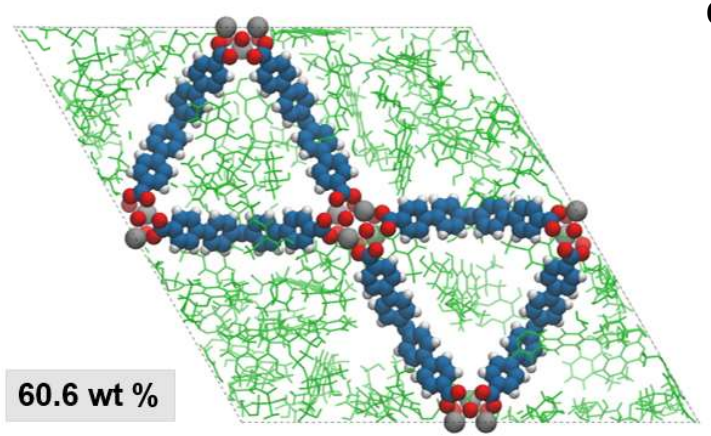

b.

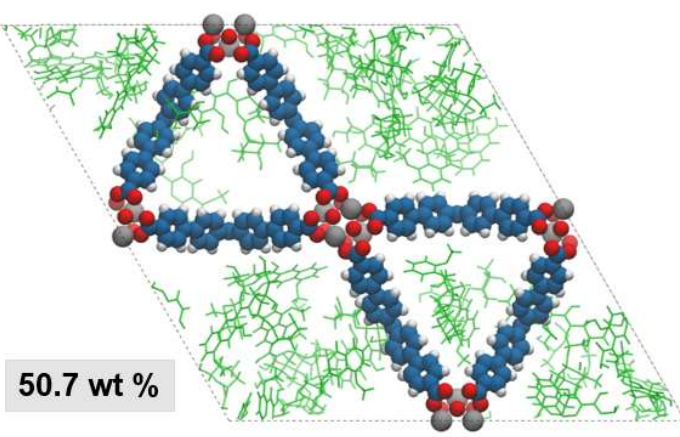

d.

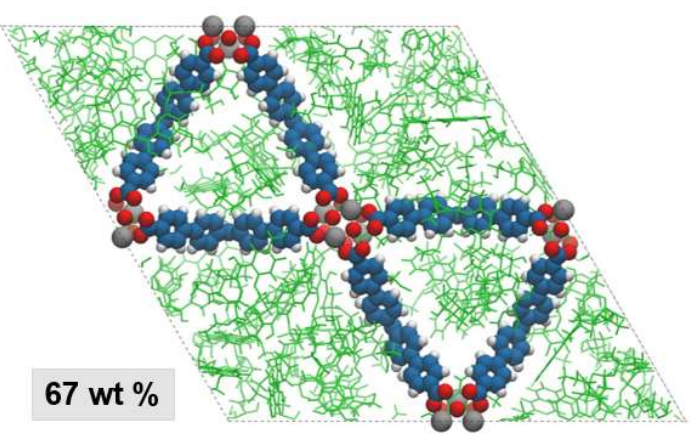

Figure S49. Snapshots of GCMC simulations showing DOX (in green) loading inside PCN-128. a. 33.9 wt $\%$, b. $50.7 \mathrm{wt} \%$, c. $60.6 \mathrm{wt} \%$, and d. $67 \mathrm{wt} \%$. For the framework, $\mathrm{C}$ atoms are in blue, $\mathrm{H}$ atoms in white, $\mathrm{O}$ atoms in red, and $\mathrm{Zr}$ atoms in grey. 
a.

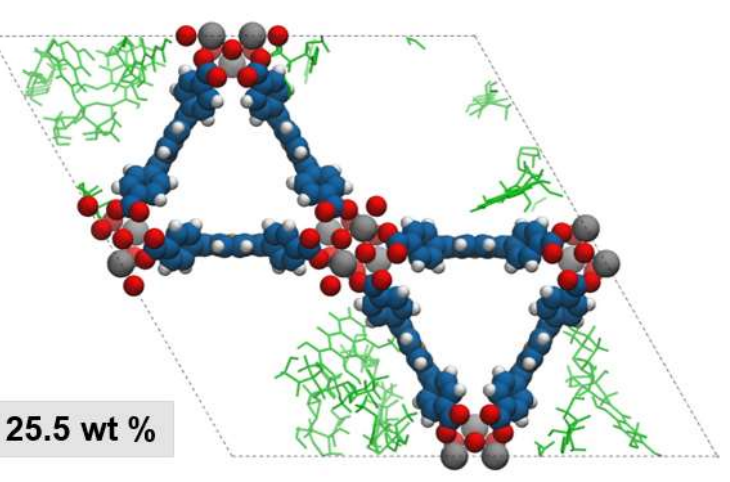

C.

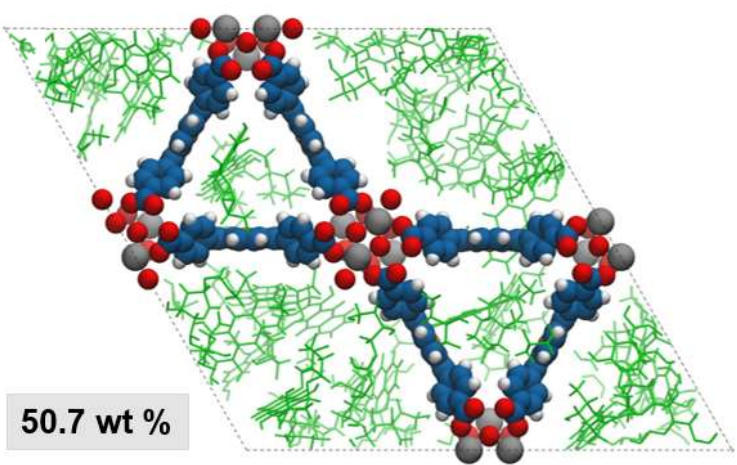

b.

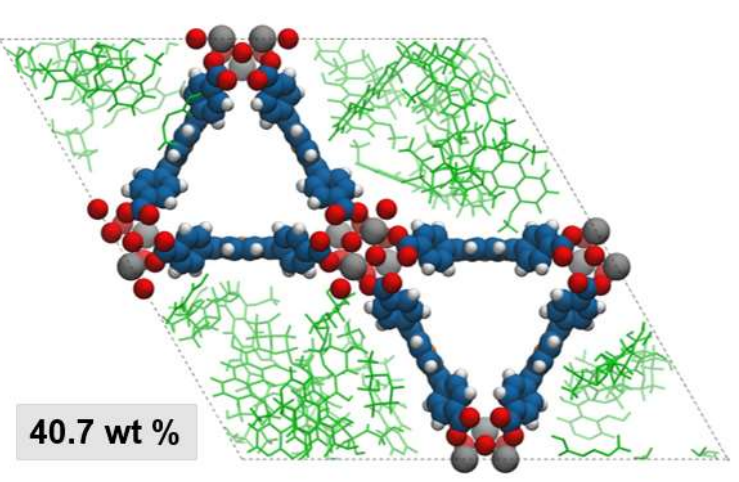

d.

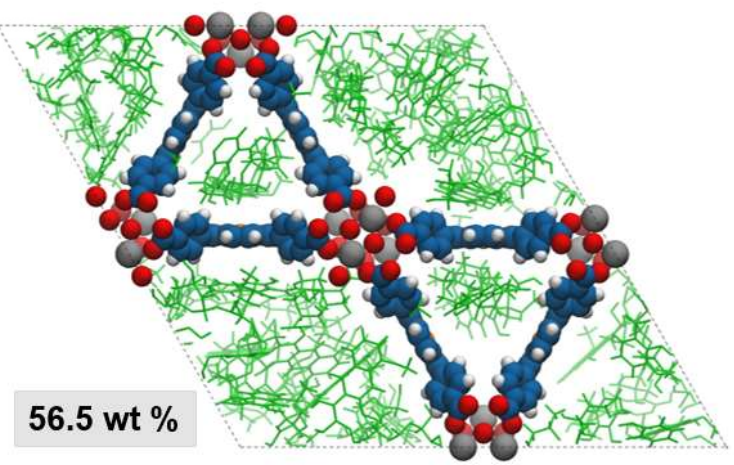

Figure S50. Snapshots of GCMC simulations showing DOX (in green) loading inside PCN-222. a. 25.5 wt\%, b. $40.7 \mathrm{wt} \%$, c. $50.7 \mathrm{wt} \%$, and d. $56.5 \mathrm{wt} \%$. For the framework, $\mathrm{C}$ atoms are in blue, $\mathrm{H}$ atoms in white, $\mathrm{N}$ atoms in orange, $\mathrm{O}$ atoms in red, and $\mathrm{Zr}$ atoms in grey. 


\section{S6. In Vitro evaluation of nanoMOFs}

Gating strategy for flow cytometric analysis of nanoMOFs association. Representative dot-plots of HeLa cells were plotted forward versus side scatter using side scatter on a linear scale, with a large gate drawn to exclude debris from the following analysis. Single stain compensations were used to identify the appropriate emission channel of each nanoMOF. Then the single-cell population was plotted against APC channel and Violet 2 channel to identify the percentage of cells associated with nanoMOF at each time point.

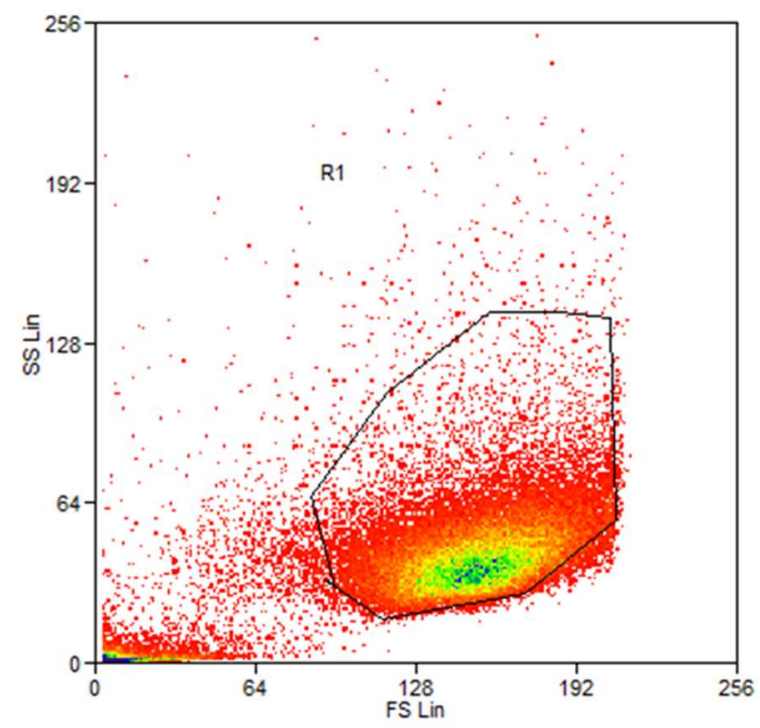

PCN-128 and PCN-128@PEG-PO
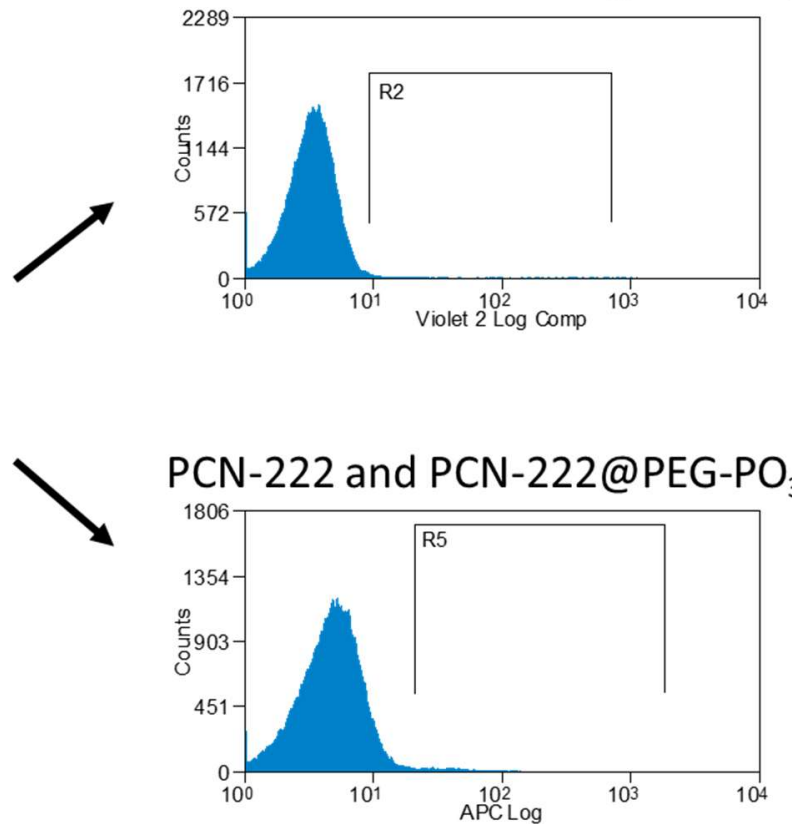

Figure S51. Example analysis of PCN-128, PCN-128@PEG-PO 3, PCN-222 and PCN-222@PEG-PO 3 using flow cytometry. 

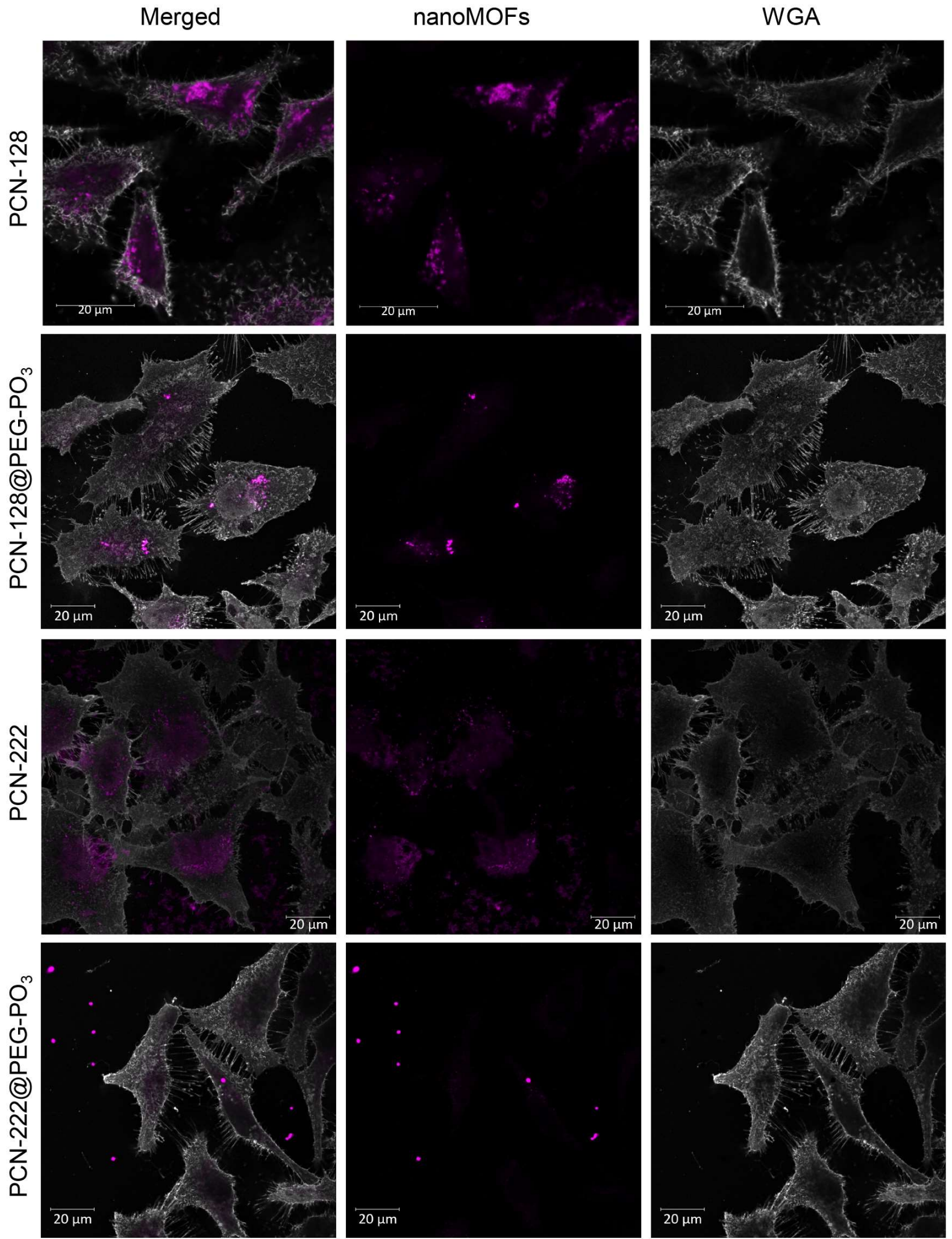

Figure S52. 2D confocal images of PCN-128, PCN-128@PEG-PO ${ }_{3}$, PCN-222 and PCN-222@PEG-PO ${ }_{3}$

HeLa cells were treated with $100 \mu \mathrm{g} / \mathrm{mL}$ for $24 \mathrm{~h}$ before they were fixed and stained with wheat germ agglutinin (WGA, colored in white), nanoMOFs in violet with merged images of both channels on the left panel. 
Table S1. Comparison of the post-synthetic methods.

\begin{tabular}{|c|c|c|c|c|}
\hline MOF & Polymer & Catalyst & Solvent & Temperature \\
\hline UiO-66 & PEG-propargyl & Cul & DCM & r.t. ${ }^{24}$ \\
\hline MIL-100 & Acryl-PEG or acryl-HA-PEG & $\begin{array}{l}\text { Iron } \\
\text { powder }\end{array}$ & $\mathrm{H}_{2} \mathrm{O}$ & r.t. 25 \\
\hline $\begin{array}{l}\text { UiO-66, } 67,68, \text { PCN-222, } \\
223,224, \text { MIL-101 }\end{array}$ & $\begin{array}{l}\text { Phosphate-terminal } \\
\text { oligonucleotides }\end{array}$ & -- & $\mathrm{H}_{2} \mathrm{O}$ & r.t. ${ }^{26}$ \\
\hline Zr-MOF & $\begin{array}{l}\text { Phosphate-terminal DNA } \\
\text { aptamer }\end{array}$ & -- & $\mathrm{DMF} / \mathrm{H}_{2} \mathrm{O}$ & r.t. $^{27}$ \\
\hline PCN-222, NU-1000 & Phosphate-terminal DNA & -- & $\mathrm{H}_{2} \mathrm{O}$ & r.t. ${ }^{28}$ \\
\hline UiO-66, UiO-67, BUT-30 & $\begin{array}{l}\text { 1,2-dioleoyl-sn-glycero-3- } \\
\text { phosphate }\end{array}$ & -- & $\mathrm{CHCl}_{3}$ & r.t. 29 \\
\hline UiO-66, PCN-222 & $n$-octadecylphosphonic acid & -- & $\mathrm{EtOH}$ & r.t. 30 \\
\hline
\end{tabular}

Table S2. ICP-OES analysis of MOF@PEG-PO3.

\begin{tabular}{cccc}
\hline Sample & $\begin{array}{c}\mathbf{P} \\
(\mathbf{m g} / \mathbf{L})\end{array}$ & $\begin{array}{c}\mathbf{Z r} \\
\mathbf{( m g / L )}\end{array}$ & $\begin{array}{c}\text { PEG loading } \\
\text { (wt\%) }\end{array}$ \\
\hline PEGylation 2 h & 0.046 & 5.114 & 27.8 \\
PEGylation 4 h & 0.058 & 4.972 & 33.3 \\
PEGylation 12 $\mathrm{h}$ & 0.071 & 5.979 & 33.6 \\
PEGylation 16 h & 0.057 & 4.926 & 32.9 \\
UiO-66@PEG-PO 3 & 0.038 & 3.939 & 37.7 \\
MOF-808@PEG-PO & 0.044 & 4.605 & 38.5 \\
NU-901@PEG-PO 3 & 0.042 & 3.700 & 30.6 \\
PCN-128@PEG-PO3 & 0.057 & 3.241 & 34.1 \\
\hline
\end{tabular}

Table $\mathrm{S} 2$ shows the mass ratio between $\mathrm{mPEG}-\mathrm{PO}_{3}$ and MOF. The ideal $\mathrm{N}_{2}$ uptake is calculated based on the assumption that the PEGylation only occurs at the external surface, which is equal to (mass ratio) $\times$ actual $\mathrm{N}_{2}$ uptake of parent MOFs.

Table S3. $\mathrm{N}_{2}$ uptake at $77 \mathrm{~K}$.

\begin{tabular}{|c|c|c|c|}
\hline Sample & $\begin{array}{c}\text { Actual } \mathrm{N}_{2} \text { uptake at } P I P_{0} \\
=0.8 \\
\left(\mathrm{~cm}^{3} \mathrm{~g}^{-1}, \mathrm{STP}\right)\end{array}$ & $\begin{array}{c}\text { Ideal } N_{2} \text { uptake at } \\
P I P_{0}=0.8 \\
\left(\mathrm{~cm}^{3} \mathrm{~g}^{-1}, \mathrm{STP}\right)\end{array}$ & $\begin{array}{c}\text { Actual uptake/ldeal } \\
\text { uptake (\%) }\end{array}$ \\
\hline PCN-222 & 526 & -- & - \\
\hline PEGylation $2 \mathrm{~h}$ & 348 & 380 & 91.6 \\
\hline PEGylation $4 \mathrm{~h}$ & 124 & 351 & 35.3 \\
\hline PEGylation $12 \mathrm{~h}$ & 121 & 349 & 34.7 \\
\hline PEGylation $16 \mathrm{~h}$ & 129 & 353 & 36.5 \\
\hline
\end{tabular}


Table S4. Experimental, simulated drug loading (max) and the related BET surface area.

\begin{tabular}{cccc}
\hline Sample & Experimental loading (wt\%) & $\begin{array}{c}\text { Simulated loading } \\
(\mathbf{w t} \mathbf{\%})\end{array}$ & BET ${\text { area }\left(\mathbf{m}^{2} \mathbf{g}^{-1}\right)}^{-1}$ \\
\hline DOX@UiO-66 & 17.6 & 0 & 873 \\
DOX@MOF-808 & 14.4 & 48.7 & 771 \\
DOX@NU-901 & 22.2 & 34.6 & 987 \\
DOX@PCN-128 & 18.1 & 67.0 & 541 \\
DOX@PCN-222 & 23.2 & 56.5 & 855 \\
\hline DOX@66@PEG-PO & 15.1 & - & 292 \\
DOX@808@PEG-PO & 13.4 & -- & 265 \\
DOX@901@PEG-PO & 20.2 & -- & 198 \\
DOX@128@PEG-PO & 13.6 & -- & 69 \\
DOX@222@PEG-PO & 15.5 & -- & 145 \\
\hline
\end{tabular}

Table S5. Atom numbers and their corresponding OPLS-AA atom types, along with the Lennard-Jones parameters and charges for the different atoms of the DOX molecule (see below figure).

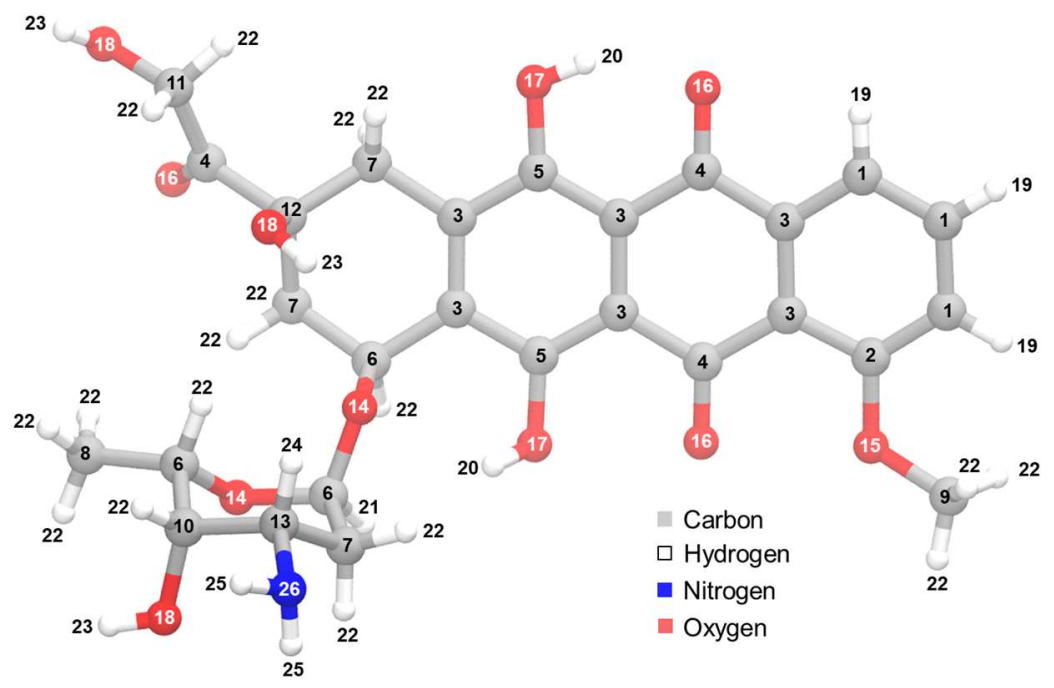

\begin{tabular}{ccccc}
\hline Number & Atom type & ${\boldsymbol{\varepsilon ~} \mathbf{k}_{\mathbf{b}}^{-1}(\mathbf{K})}^{-}$ & $\boldsymbol{\sigma}(\mathbf{A})$ & $\mathbf{q}(\mathbf{e})$ \\
\hline 1 & CA & 35.22 & 3.55 & -0.115 \\
2 & CA & 35.22 & 3.55 & 0.17 \\
3 & CA & 35.22 & 3.55 & 0 \\
4 & C_2 & 52.83 & 3.75 & 0.47 \\
5 & CA & 35.22 & 3.55 & 0.15 \\
6 & CT & 33.21 & 3.5 & 0.17 \\
7 & CT & 33.21 & 3.5 & -0.12 \\
8 & CT & 33.21 & 3.5 & -0.18 \\
9 & CT & 33.21 & 3.5 & -0.065 \\
10 & CT & 33.21 & 3.5 & 0.205 \\
11 & CT & 33.21 & 3.5 & -0.12 \\
12 & CT & 33.21 & 3.5 & -0.265 \\
13 & CT & 33.21 & 3.5 & 0.12 \\
\hline
\end{tabular}




\begin{tabular}{lcccc}
\hline 14 & OS & 70.45 & 2.9 & -0.3 \\
15 & OS & 70.45 & 2.9 & -0.285 \\
16 & O_2 & 105.67 & 2.96 & -0.47 \\
17 & OH & 85.54 & 3.07 & -0.585 \\
18 & OH & 85.54 & 3.12 & -0.683 \\
19 & HA & 15.09 & 2.42 & 0.115 \\
20 & HO & 0 & 0 & 0.435 \\
21 & HC & 15.09 & 2.5 & 0.03 \\
22 & HC & 15.09 & 2.5 & 0.06 \\
23 & HO & 0 & 0 & 0.418 \\
24 & HC & 7.548 & 2.5 & 0.06 \\
25 & H & 0 & 0 & 0.36 \\
26 & NT & 85.54 & 3.3 & -0.9 \\
\hline
\end{tabular}

Table S6. Lennard-Jones parameters for the atoms in the framework. Column 1 lists the atom type; columns 2 and 3 list the Lennard-Jones parameters for the corresponding atom types, $\sigma$ in $\AA$ and $\varepsilon / K_{b}$ in $\mathrm{K}$, taken from the DREIDING force field, and for $\mathrm{Zr}$, which is not present in the DREIDING force field, from the UFF (marked with an asterisk).

\begin{tabular}{ccc}
\hline \multirow{2}{*}{ Atom } & \multicolumn{2}{c}{ LJ Parameters } \\
\cline { 2 - 3 } & $\boldsymbol{\sigma}(\mathbf{A})$ & $\boldsymbol{\varepsilon} / \mathbf{k}_{\mathbf{B}}(\mathbf{K})$ \\
\hline $\mathrm{C}$ & 3.473 & 47.888 \\
$\mathrm{H}$ & 2.846 & 7.654 \\
$\mathrm{~N}$ & 3.263 & 38.975 \\
$\mathrm{O}$ & 3.033 & 48.190 \\
$\mathrm{Zr}^{*}$ & 2.783 & 34.745 \\
\hline
\end{tabular}




\section{S7. References}

1. Le Ouay, B.; Watanabe, C.; Mochizuki, S.; Takayanagi, M.; Nagaoka, M.; Kitao, T.; Uemura, T., Selective sorting of polymers with different terminal groups using metal-organic frameworks. Nat. Commun. 2018, 9, 3635.

2. Hasse, H.; Kany, H. P.; Tintinger, R.; Maurer, G., Osmotic Virial Coefficients of Aqueous Poly(ethylene glycol) from Laser-Light Scattering and Isopiestic Measurements. Macromolecules 1995, 28, 3540-3552.

3. Feng, D.; Gu, Z. Y.; Li, J. R.; Jiang, H. L.; Wei, Z.; Zhou, H. C., Zirconium-metalloporphyrin PCN222: mesoporous metal-organic frameworks with ultrahigh stability as biomimetic catalysts. Angew. Chem. Int. Ed. 2012, 51, 10307-10310.

4. Wilmer, C. E.; Kim, K. C.; Snurr, R. Q., An Extended Charge Equilibration Method. J. Phys. Chem. Lett. 2012, 3, 2506-2511.

5. Plimpton, S., Fast Parallel Algorithms for Short-Range Molecular Dynamics. J. Comput. Phys. 1995, 117, 1-19.

6. Rappe, A. K.; Casewit, C. J.; Colwell, K. S.; Goddard, W. A.; Skiff, W. M., UFF, a full periodic table force field for molecular mechanics and molecular dynamics simulations. J. Am. Chem. Soc. 1992, 114, 10024-10035.

7. Evans, D. J.; Holian, B. L., The Nose-Hoover thermostat. J. Chem. Phys. 1985, 83, 4069-4074.

8. Humphrey, W.; Dalke, A.; Schulten, K., VMD: Visual molecular dynamics. J. Mol. Graph. 1996, 14, 33-38.

9. Markopoulou, P.; Panagiotou, N.; Li, A.; Bueno-Perez, R.; Madden, D.; Buchanan, S.; FairenJimenez, D.; Shiels, P. G.; Forgan, R. S., Identifying Differing Intracellular Cargo Release Mechanisms by Monitoring In Vitro Drug Delivery from MOFs in Real Time. Cell Rep. Phys. Sci. 2020, 1, 100254.

10. Jorgensen, W. L.; Maxwell, D. S.; Tirado-Rives, J., Development and Testing of the OPLS AllAtom Force Field on Conformational Energetics and Properties of Organic Liquids. J. Am. Chem. Soc. 1996, 118, 11225-11236.

11. Dubbeldam, D.; Calero, S.; Ellis, D. E.; Snurr, R. Q., RASPA: molecular simulation software for adsorption and diffusion in flexible nanoporous materials. Mol. Simul. 2015, 42, 81-101.

12. Moghadam, P. Z.; Li, A.; Wiggin, S. B.; Tao, A.; Maloney, A. G. P.; Wood, P. A.; Ward, S. C.; Fairen-Jimenez, D., Development of a Cambridge Structural Database Subset: A Collection of Metal-Organic Frameworks for Past, Present, and Future. Chem. Mater. 2017, 29, 2618-2625.

13. Mayo, S. L.; Olafson, B. D.; Goddard, W. A., DREIDING: a generic force field for molecular simulations. J. Phys. Chem. 1990, 94, 8897-8909.

14. Wei, Z.; Gu, Z. Y.; Arvapally, R. K.; Chen, Y. P.; McDougald, R. N., Jr.; Ivy, J. F.; Yakovenko, A. A.; Feng, D.; Omary, M. A.; Zhou, H. C., Rigidifying fluorescent linkers by metal-organic framework formation for fluorescence blue shift and quantum yield enhancement. J. Am. Chem. Soc. 2014, 136, 8269-8276.

15. Noh, H.; Kung, C.-W.; Islamoglu, T.; Peters, A. W.; Liao, Y.; Li, P.; Garibay, S. J.; Zhang, X.; DeStefano, M. R.; Hupp, J. T.; Farha, O. K., Room Temperature Synthesis of an 8-Connected Zr-Based Metal-Organic Framework for Top-Down Nanoparticle Encapsulation. Chem. Mater. 2018, 30, 2193-2197.

16. Boyer, J. C.; Manseau, M. P.; Murray, J. I.; van Veggel, F. C., Surface modification of upconverting $\mathrm{NaYF}_{4}$ nanoparticles with PEG-phosphate ligands for NIR $(800 \mathrm{~nm})$ biolabeling within the biological window. Langmuir 2010, 26, 1157-1164.

17. Naszalyi Nagy, L.; Mihaly, J.; Polyak, A.; Debreczeni, B.; Csaszar, B.; Szigyarto, I. C.; Wacha, A.; Czegeny, Z.; Jakab, E.; Klebert, S.; Drotar, E.; Dabasi, G.; Bota, A.; Balogh, L.; Kiss, E., Inherently fluorescent and porous zirconia colloids: preparation, characterization and drug adsorption studies. J. Mater. Chem. B 2015, 3, 7529-7537.

18. Uemura, T.; Yanai, N.; Watanabe, S.; Tanaka, H.; Numaguchi, R.; Miyahara, M. T.; Ohta, Y.; Nagaoka, M.; Kitagawa, S., Unveiling thermal transitions of polymers in subnanometre pores. Nat. Commun. 2010, 1, 83.

19. Xie, Z.; Wang, B.; Yang, Z.; Yang, X.; Yu, X.; Xing, G.; Zhang, Y.; Chen, L., Stable 2D Heteroporous Covalent Organic Frameworks for Efficient lonic Conduction. Angew. Chem. Int. Ed. 2019, 58, 15742-15746. 
20. Zhao, G.; Tong, L.; Cao, P.; Nitz, M.; Winnik, M. A., Functional PEG-PAMAM-tetraphosphonate capped $\mathrm{NaLnF}_{4}$ nanoparticles and their colloidal stability in phosphate buffer. Langmuir 2014, 30, 6980-6989.

21. Li, S.; Amat, D.; Peng, Z.; Vanni, S.; Raskin, S.; De Angulo, G.; Othman, A. M.; Graham, R. M.; Leblanc, R. M., Transferrin conjugated nontoxic carbon dots for doxorubicin delivery to target pediatric brain tumor cells. Nanoscale 2016, 8, 16662-16669.

22. Li, S. Y.; Zhao, L. P.; Zheng, R. R.; Fan, G. L.; Liu, L. S.; Zhou, X.; Chen, X. T.; Qiu, X. Z.; Yu, X. Y.; Cheng, H., Tumor Microenvironment Adaptable Nanoplatform for $\mathrm{O}_{2}$ Self-Sufficient Chemo/Photodynamic Combination Therapy. Part. Part. Syst. Charact. 2020, 37, 1900496.

23. Osterrieth, J.; Rampersad, J.; Madden, D. G.; etc. How Reproducible Are Surface Areas Calculated from the BET Equation? May 26, 2021. ChemRxiv. DOI: 10.26434/chemrxiv.14291644.v2 (accessed 2021-07-15).

24. Abanades Lazaro, I.; Haddad, S.; Sacca, S.; Orellana-Tavra, C.; Fairen-Jimenez, D.; Forgan, R. S., Selective Surface PEGylation of UiO-66 Nanoparticles for Enhanced Stability, Cell Uptake, and pH-Responsive Drug Delivery. Chem 2017, 2, 561-578.

25. Gimenez-Marques, M.; Bellido, E.; Berthelot, T.; Simon-Yarza, T.; Hidalgo, T.; Simon-Vazquez, R.; Gonzalez-Fernandez, A.; Avila, J.; Asensio, M. C.; Gref, R.; Couvreur, P.; Serre, C.; Horcajada, P., GraftFast Surface Engineering to Improve MOF Nanoparticles Furtiveness. Small 2018, 14, 1801900.

26. Wang, S.; McGuirk, C. M.; Ross, M. B.; Wang, S.; Chen, P.; Xing, H.; Liu, Y.; Mirkin, C. A., General and Direct Method for Preparing Oligonucleotide-Functionalized Metal-Organic Framework Nanoparticles. J. Am. Chem. Soc. 2017, 139, 9827-9830.

27. Liu, Y.; Hou, W.; Xia, L.; Cui, C.; Wan, S.; Jiang, Y.; Yang, Y.; Wu, Q.; Qiu, L.; Tan, W., ZrMOF nanoparticles as quenchers to conjugate DNA aptamers for target-induced bioimaging and photodynamic therapy. Chem. Sci., 2018, 9, 7505-7509.

28. Wang, S.; Chen, Y.; Wang, S.; Li, P.; Mirkin, C. A.; Farha, O. K., DNA-Functionalized MetalOrganic Framework Nanoparticles for Intracellular Delivery of Proteins. J. Am. Chem. Soc. 2019, 141, 2215-2219.

29. Wang, S.; Morris, W.; Liu, Y.; McGuirk, C. M.; Zhou, Y.; Hupp, J. T.; Farha, O. K.; Mirkin, C. A., Surface-Specific Functionalization of Nanoscale Metal-Organic Frameworks. Angew. Chem. Int. Ed. 2015, 54, 14738-14742.

30. Sun, Y.; Sun, Q.; Huang, H.; Aguila, B.; Niu, Z.; Perman, J. A.; Ma, S., A molecular-level superhydrophobic external surface to improve the stability of metal-organic frameworks. $J$. Mater. Chem. A, 2017, 5, 18770-18776. 